\title{
A cyclic extension of the earthquake flow I
}

\author{
FRANCESCO BONSANTE \\ GABRIELE MONDELLO \\ JEAN-MARC SCHLENKER
}

Let $\mathcal{T}$ be Teichmüller space of a closed surface of genus at least 2 . We describe an action of the circle on $\mathcal{T} \times \mathcal{T}$, which limits to the earthquake flow when one of the parameters goes to a measured lamination in the Thurston boundary of $\mathcal{T}$. This circle action shares some of the main properties of the earthquake flow, for instance it satisfies an extension of Thurston's Earthquake Theorem and it has a complex extension which is analogous and limits to complex earthquakes. Moreover, a related circle action on $\mathcal{T} \times \mathcal{T}$ extends to the product of two copies of the universal Teichmüller space.

$57 \mathrm{M} 50$

\section{Introduction}

In this paper we consider a closed, oriented surface $S$ of genus at least 2 . We denote by $\mathcal{T}_{S}$, or simply by $\mathcal{T}$, the Teichmüller space of $S$, and by $\mathcal{M} \mathcal{L}_{S}$, or simply by $\mathcal{M L}$, the space of measured laminations on $S$.

\subsection{Earthquakes on hyperbolic surfaces}

Given a measured lamination $\lambda \in \mathcal{M} \mathcal{L}_{S}$, we denote by $E_{\lambda}$ the left earthquake along $\lambda$ on $S . E_{\lambda}$ is a real-analytic map from $\mathcal{T}_{S}$ to $\mathcal{T}_{S}$; see Thurston [42], Kerckhoff [20] and McMullen [28]. Recall that, in the simplest case where $\lambda$ is supported on the simple closed curve $\gamma$ with mass $a$, if $h \in \mathcal{T}_{S}$ is a hyperbolic metric on $S, E_{\lambda}(h)$ is obtained by cutting $(S, h)$ open along the minimizing geodesic homotopic to $\gamma$, rotating the left-hand side of $\gamma$ by $a$, and gluing back.

We consider here the earthquake flow, which can be defined as a map

$$
\begin{aligned}
E: \mathbb{R} \times \mathcal{T} \times \mathcal{M L} & \rightarrow \mathcal{T} \times \mathcal{M L}, \\
(t, h, \lambda) & \mapsto\left(E_{t \lambda}(h), \lambda\right) .
\end{aligned}
$$


We call $E_{t}$ the corresponding map from $\mathcal{T} \times \mathcal{M L}$ to $\mathcal{T} \times \mathcal{M L}$, and will also use the notation $E_{\lambda}(h):=E_{1}(h, \lambda)$.

Earthquakes have a number of interesting properties, of which we can single the following.

(1) The earthquake flow defined above is indeed a flow: for all $s, t \in \mathbb{R}$, we have $E_{s} \circ E_{t}=E_{s+t}$.

(2) Thurston's Earthquake Theorem (see [20]): for any $h, h^{\prime} \in \mathcal{T}$, there is a unique $\lambda \in \mathcal{M L}$ such that $E_{\lambda}(h)=h^{\prime}$.

(3) For fixed $\lambda \in \mathcal{M} \mathcal{L}_{S}$ and $h \in \mathcal{T}_{S}$, the map

$$
\begin{aligned}
\mathbb{R} & \rightarrow \mathcal{T}_{S}, \\
t & \mapsto E_{-t \lambda}(h),
\end{aligned}
$$

extends to a holomorphic map on a simply connected domain in $\mathbb{C}$ containing all complex numbers with nonnegative imaginary part; see [28]. This defines the notion of "complex earthquake".

(4) When considered on imaginary numbers, complex earthquakes correspond to conformal grafting maps $g r$, which are related to complex earthquakes by $\operatorname{gr}(t, \lambda)(h)=E_{i t \lambda}(h)$. The conformal grafting map $g r: \mathbb{R}_{\geq 0} \times \mathcal{T} \times \mathcal{M L} \rightarrow \mathcal{T}$ is actually obtained by composing the projective grafting map

$$
G r: \mathbb{R}_{\geq 0} \times \mathcal{T} \times \mathcal{M L} \rightarrow \mathcal{P},
$$

where $\mathcal{P}$ is the space of complex projective structures on $S$, with the forgetful map $\mathcal{P} \rightarrow \mathcal{T}$ sending a $\mathbb{C} \mathbb{P}^{1}$-structure to the underlying complex structure.

(5) Thurston (see Kulkarni and Pinkall [23]) proved that, for all $s>0, G r_{s}$ is a homeomorphism from $\mathcal{T} \times \mathcal{M L}$ to $\mathcal{P}$.

\subsection{The landslide flow}

We introduce a flow on Teichmüller space which in a way extends the earthquake flow, and which shares the properties described above. The corresponding deformations are "smoother" than earthquakes, but earthquakes are limits in a natural sense. This motivates the term "landslide" that we use here. This deformation depends not on a measured lamination but rather on a hyperbolic metric $h^{\star} \in \mathcal{T}$ and it determines a flow

$$
\begin{aligned}
L: \mathcal{T} \times \mathcal{T} \times S^{1} & \rightarrow \mathcal{T} \times \mathcal{T}, \\
\left(h, h^{\star}, e^{i \theta}\right) & \mapsto L_{e^{i \theta}, h^{\star}}(h) .
\end{aligned}
$$


We denote by $L_{e^{i \theta}}: \mathcal{T} \times \mathcal{T} \rightarrow \mathcal{T} \times \mathcal{T}$ the corresponding map seen as depending on the parameter $e^{i \theta}$.

We will also use the notation $L^{1}$ for the composition of $L$ with the projection on the first factor, so that $L^{1}$ is a map from $S^{1} \times \mathcal{T} \times \mathcal{T}$ to $\mathcal{T}$. $L_{e^{i \theta}}^{1}$ will denote the same map, considered as depending on the parameter $e^{i \theta} \in S^{1}$, so it is a map from $\mathcal{T} \times \mathcal{T}$ to $\mathcal{T}$. Thus $L_{e^{i \theta}}^{1}$ is the analog of the earthquake map $E: \mathcal{T} \times \mathcal{M L} \rightarrow \mathcal{T}$.

When the metrics $h$ and $h^{\star}$ are fixed, we can consider the image of the map $S^{1} \ni e^{i \theta} \mapsto$ $L_{e^{i \theta}}^{1}\left(h, h^{\star}\right) \in \mathcal{T}$ as a circle in Teichmüller space in which $h$ and $h^{\star}$ are antipodal points. When $h^{\star}$ converges (projectively) to a measured lamination $\lambda$ at the Thurston boundary of Teichmüller space, such a circle converges to the earthquake line $E_{t \lambda}(h)$. A more precise statement, Theorem 1.12, can be found below.

This "landslide flow" shares the main properties of the earthquake flow recalled above:

(1) $L$ is a flow on $\mathcal{T} \times \mathcal{T}$-depending on the definition: checking this can be nontrivial; see Theorem 1.8.

(2) We prove an analog of Thurston's Earthquake Theorem; see Theorem 1.15.

(3) For fixed $h, h^{\star} \in \mathcal{T}$, the map $L_{\bullet}\left(h, h^{\star}\right): S^{1} \rightarrow \mathcal{T}$ extends to a holomorphic map from the closed unit disk $\bar{\Delta}$ to $\mathcal{T}$; see Theorem 5.1. This defines the "complex landslide" which are analogs of the "complex earthquakes".

(4) For $r \in(0,1)$, the complex landslide $L_{r}$ corresponds to what we call here "smooth grafting", which is analogous to grafting in our context and we denote by $s g r_{r}: \mathcal{T} \times \mathcal{T} \rightarrow \mathcal{T}$ the map defined as $L_{r}: \mathcal{T} \times \mathcal{T} \rightarrow \mathcal{T} \times \mathcal{T}$ followed by projection on the first factor. It is obtained by composing a map $S G r:(0,1) \times \mathcal{T} \times \mathcal{T} \rightarrow \mathcal{P}$ with the natural projection from $\mathcal{P}$ to $\mathcal{T}$.

(5) For all $r \in(0,1)$, the map $\operatorname{SGr}(r, \bullet, \bullet):(0,1) \times \mathcal{T} \times \mathcal{T} \rightarrow \mathcal{P}$ is a homeomorphism.

Our notation means that we parameterize the complex landslides using the unit disk in $\mathbb{C}$ rather than the upper half-plane as is customary for complex earthquakes. This notation is clearly equivalent but using the disk appears more natural in the context of the landslides considered here.

Considered as a circle action on $\mathcal{T} \times \mathcal{T}$, the flow $L$ extends to a circle action on the universal Teichmüller space; see Section 8.

\subsection{Example: landslides for flat tori}

The main properties of the landslide flow can be better understood by considering the Teichmüller space of the torus, that is, the space $\mathcal{T}_{1}$ of flat metrics of area 1 on the 
torus, considered up to isotopy. $\mathcal{T}_{1}$ is classically identified with the hyperbolic plane, and the mapping-class group of the torus with $\mathrm{SL}_{2} \mathbb{Z}$.

It is natural to define on $\mathcal{T}_{1}$ the analog of the Weil-Petersson metric, and the analog of the Teichmüller metric, and both correspond to the hyperbolic metric on $\mathbb{H}^{2}$ (up to a factor $\sqrt{2}$ ); see Appendix A.

It is also well-known that earthquakes act on $\mathcal{T}_{1}$ as horocyclic flows on $\mathbb{H}^{2}$. In Appendix A it is proven that the landslide flow defined here acts on $\mathcal{T}_{1} \times \mathcal{T}_{1}$ by sending $\left(h, h^{\star}, e^{i \theta}\right)$ to $\left(h_{\theta}, h_{\theta}^{\star}\right)$, where $h_{\theta}$ and $h_{\theta}^{\star}$ are obtained by rotating $h$ and $h^{\star}$ by an angle $\theta$ around the center $c$ of the segment from $h$ to $h^{\star}$ in $\mathcal{T}_{1}$. It is quite easy to see that this flow limits to the earthquake flow, as happens in the higher genus case (see Theorem 1.12 below).

The analog of the Earthquake theorem for the landslide flow is then a simple statement on the existence of a unique circle in $\mathbb{H}^{2}$ going through two points $h, h^{\star}$ on which they are separated by a fixed angle $\theta$. The other properties of the landslide flow presented here, when considered for flat metrics on the torus, similarly have a simple interpretation in terms of elementary properties of the hyperbolic plane.

\subsection{Harmonic maps and the landslide flow}

Consider two hyperbolic metrics $c$ and $h$ on $S$. A map $f:(S, c) \rightarrow(S, h)$ is said to be harmonic if it is a critical point of the energy $E$. The energy considered here is

$$
E(f)=\frac{1}{2} \int_{S}\|d f\|^{2} \omega_{c},
$$

where $\omega_{c}$ is the area element of $(S, c)$ and the norm $\|\cdot\|$ is defined with respect to the metric $c$ on the domain and the metric $h$ on the target. Although it is not immediately apparent in this definition, this notion of harmonicity is conformally invariant on the domain, because changing $c$ by a factor $\lambda$ multiplies $\omega_{c}$ by $\lambda$ but divides $\|d f\|^{2}$ by the same factor. So we can regard $c$ as a conformal structure on $S$ rather than a metric.

Theorem 1.1 (Sampson [33], Eells and Sampson [12], Hartman [15], Al'ber [1], Schoen and Yau [39]) Let $c$ be a conformal class on $S$, and let $h \in \mathcal{T}$ be a hyperbolic metric. There is a unique harmonic map $f:(S, c) \rightarrow(S, h)$ isotopic to the identity. Moreover, $f$ is a diffeomorphism.

Consider a $C^{1}$ map $f:\left(S, h^{\#}\right) \rightarrow(S, h)$, where $h^{\#}$ is a metric in the conformal class of $c$. The Hopf differential $\Phi(f)$ of $f$ is a quadratic differential that measures the traceless part of the pullback of $h$ by $f$ and it is defined by the formula

$$
f^{*} h=e h^{\#}+\Phi+\bar{\Phi}
$$


where $e=\frac{1}{2} \operatorname{tr}_{h^{*}}\left(f^{*} h\right)$. If $f$ is harmonic, then $\Phi$ is holomorphic. For $f \in C^{2}$ with $d f$ of maximal rank everywhere, the converse also holds; see [33]. It follows from its definition that $\Phi(f)$ is invariant under conformal changes of the metric $h^{\#}$ on $S$.

Conversely, given a holomorphic quadratic differential $\Phi$ on $(S, c)$, there exists a unique hyperbolic metric $h$ on $S$ such that the identity map $(S, c) \rightarrow(S, h)$ is harmonic with Hopf differential $\Phi$; see [33] and Wolf [43].

This leads to the definition of a flow on $\mathcal{T}$ depending on a "center" $c \in \mathcal{T}$.

Definition 1.2 Let $c, h \in \mathcal{T}$ and let $e^{i \theta} \in S^{1}$. We define $R_{c, e^{i \theta}}(h)$ as the (unique) hyperbolic metric $h^{\prime}$ on $S$ such that, if $f:(S, c) \rightarrow(S, h)$ and $f^{\prime}:(S, c) \rightarrow\left(S, h^{\prime}\right)$ are the harmonic maps isotopic to the identity, then

$$
\Phi\left(f^{\prime}\right)=e^{i \theta} \Phi(f) .
$$

This simple definition is strongly related to the flow $L$ mentioned above, but the relation is not obvious (see Corollary 1.11), and directly using the definition of $R$ given here is not convenient. For this reason we give below a different definition of $L$, which is more geometric, less directly accessible, but leads to straightforward arguments.

There is another, superficially similar flow on Teichmüller space, the elliptic flow defined by one of us (Mondello); see [31]. There are only limited similarities between the two flows, as should be clear from the sequel.

\subsection{Minimal Lagrangian maps between hyperbolic surfaces}

The constructions considered here depend strongly on the notion of minimal Lagrangian maps between hyperbolic surfaces. Recall that, given two hyperbolic metrics $h$ and $h^{\star}$ on $S$, a diffeomorphism $m:(S, h) \rightarrow\left(S, h^{\star}\right)$ is minimal Lagrangian if

- it is area-preserving and orientation-preserving,

- its graph is a minimal surface in $\left(S \times S, h \oplus h^{\star}\right)$.

Theorem 1.3 (Schoen [38], Labourie [25]) Let $h, h^{\star}$ be two hyperbolic metrics on $S$. There exists a unique minimal Lagrangian diffeomorphism $m:(S, h) \rightarrow\left(S, h^{\star}\right)$ isotopic to the identity.

Minimal Lagrangian maps actually have a description in terms of hyperbolic surfaces only, as follows (see eg [25]). 
Proposition 1.4 If $m:(S, h) \rightarrow\left(S, h^{\star}\right)$ is minimal Lagrangian, then there exists a bundle map $b: T S \rightarrow T S$ such that

(i) $m^{*} h^{\star}=h(b \bullet, b \bullet)$;

(ii) $b$ is self-adjoint for $h$;

(iii) $b$ has determinant 1 ;

(iv) $b$ satisfies the Codazzi equation: $d^{\nabla} b=0$, where $\nabla$ is the Levi-Civita connection of $h$.

Conversely, if $m: S \rightarrow S$ is a diffeomorphism for which there exists a map $b$ satisfying those properties, then $m$ is minimal Lagrangian.

Corollary 1.5 Let $h, h^{\star}$ be two hyperbolic metrics on $S$. There exists a unique bundle morphism $b: T S \rightarrow T S$ which is self-adjoint for $h$, of determinant equal to 1 everywhere, satisfying the Codazzi equation $d^{\nabla} b=0$, where $\nabla$ is the Levi-Civita connection of $h$, and such that $h(b \bullet, b \bullet)$ is isometric to $h^{\star}$ by a diffeomorphism isotopic to the identity.

A consequence of this Proposition is that for any $\tau, \tau^{\star} \in \mathcal{T}$, we can realize $\tau$ and $\tau^{\star}$ as a pair of hyperbolic metrics $h$ and $h^{\star}$ (not considered up to isometries isotopic to the identity) so that $h^{\star}=h(b \bullet, b \bullet)$, where $b$ is self-adjoint for $h$, of determinant 1 , and satisfies the Codazzi equation $d^{\nabla} b=0$. A pair of metrics with this property will be called a normalized representative of $\left(\tau, \tau^{\star}\right)$. Clearly a normalized representative of $\left(\tau, \tau^{\star}\right)$ is uniquely determined up to isotopies acting diagonally on both $h$ and on $h^{\star}$. By abuse of notation, we will sometimes denote by $\left(h, h^{\star}\right)$ both a couple of normalized hyperbolic metrics and its corresponding point in $\mathcal{T} \times \mathcal{T}$.

\subsection{The landslide action on $\mathcal{T} \times \mathcal{T}$}

We now introduce the action $L$ of $S^{1}$ on $\mathcal{T} \times \mathcal{T}$. We will see below that it is strongly related to the map $R$ introduced above.

Definition 1.6 Let $h, h^{\star}$ be two hyperbolic metrics on $S$, and let $\theta \in \mathbb{R}$. We consider the bundle morphism $b: T S \rightarrow T S$ given by Corollary 1.5 , and set

$$
\beta_{\theta}:=\cos (\theta / 2) E+\sin (\theta / 2) J b,
$$

where $E: T S \rightarrow T S$ is the identity map and $J$ is the complex structure of $h$ on $S$. We then define

$$
L_{e^{i \theta}}\left(h, h^{\star}\right):=\left(h\left(\beta_{\theta} \bullet, \beta_{\theta} \bullet\right), h\left(\beta_{\theta+\pi} \bullet, \beta_{\theta+\pi} \bullet\right)\right) .
$$

Notice that by construction, $L_{1}\left(h, h^{\star}\right)=\left(h, h^{\star}\right)$, while $L_{-1}\left(h, h^{\star}\right)=\left(h^{\star}, h\right)$. 
Proposition 1.7 For all $\theta \in \mathbb{R}, h\left(\beta_{\theta} \bullet, \beta_{\theta} \bullet\right)$ is a hyperbolic metric on $S$.

Theorem 1.8 Let $h, h^{\star}$ be two hyperbolic metrics on $S$, let $\theta, \theta^{\prime} \in \mathbb{R}$. Then

$$
L_{e^{i \theta^{\prime}}}\left(L_{e^{i \theta}}\left(h, h^{\star}\right)\right)=L_{e^{i\left(\theta^{\prime}+\theta\right)}}\left(h, h^{\star}\right) .
$$

In other words, $L$ defines an action of $S^{1}$ on $\mathcal{T} \times \mathcal{T}$. We call $L$ the landslide flow, or landslide action on $\mathcal{T} \times \mathcal{T}$.

The proofs of Proposition 1.7 and of Theorem 1.8 are in Section 3.3.

\subsection{Relations to AdS geometry}

We briefly recall some properties of AdS geometry. More details can be found eg by Mess in [29], by Andersson et al in [2] and in Section 2.

The anti-de Sitter space is a Lorentz analog of hyperbolic 3-space, and it can be defined as the quadric

$$
\mathbb{A d} \mathbb{S}^{3}=\left\{x \in \mathbb{R}^{2,2} \mid\langle x, x\rangle=-1\right\},
$$

where $\mathbb{R}^{2,2}=\left(\mathbb{R}^{4},-d x_{0}^{2}-d x_{1}^{2}+d x_{2}^{2}+d x_{3}^{2}\right)$. It is a complete Lorentz manifold of constant curvature -1 with fundamental group isomorphic to $\mathbb{Z}$.

A manifold $N$ with an AdS metric_a Lorentz metric locally modeled on $\mathbb{A d} \mathbb{S}^{3}$ - is globally hyperbolic and spacially compact $(\mathrm{GHC})$ if

- $\quad N$ contains a closed space-like surface $F$,

- any inextendible time-like curve in $N$ intersects $F$ exactly once.

Moreover, $N$ is maximal globally hyperbolic and spacially compact $(M G H C)$ if it is globally hyperbolic spacially compact and $N$ is maximal for inclusion among (GHC) manifolds. The definition of a GHC or MGHC de Sitter 3-manifold is analogous.

Mess [29; 2] proved that, if $N$ is a MGHC AdS manifold and $\bar{\phi}: S \rightarrow N$ is an embedding onto a closed space-like surface $F$, then $N$ is the quotient of a convex domain $\Omega$ in $\mathbb{A d S}^{3}$ by an action of the fundamental group of $S$.

A key feature of $\mathbb{A d S}^{3}$ is that the identity component of its isometry group is isomorphic to $\mathrm{SL}_{2}(\mathbb{R}) \times \mathrm{SL}_{2}(\mathbb{R}) / \mathbb{Z}_{2}$, which is the double cover of $\mathrm{PSL}_{2}(\mathbb{R}) \times \mathrm{PSL}_{2}(\mathbb{R})$. As a consequence, the action of $\pi_{1}(S)$ on $\Omega$ decomposes as $\left(\rho_{l}, \rho_{r}\right)$, where $\rho_{l}$ and $\rho_{r}$ are morphisms from $\pi_{1}(S)$ to $\mathrm{PSL}_{2}(\mathbb{R})$. It was proved in [29] that these morphisms have maximal Euler number, so that they correspond to points in the Teichmüller space of $S$. Maximal globally hyperbolic spacially compact AdS spaces are uniquely determined by these left and right representations; see [29;2]. 
Normalized metrics can be used to produce equivariant space-like embeddings of $\widetilde{S}$ into $\mathbb{A d S}^{3}$ of constant curvature. Let us recall that an embedding $\sigma: \widetilde{S} \rightarrow \mathbb{A d} \mathbb{S}^{3}$ is space-like if it induces a Riemannian metric on $S$, which is called the first fundamental form of the embedding. Any space-like embedding determines a normal field $\tilde{v}$ such that $\langle\widetilde{v}, \widetilde{v}\rangle=-1$ and $\widetilde{v}$ is future-oriented. The shape operator of the embedding is $\widetilde{B}=\bar{\nabla} \widetilde{v}$ where $\bar{\nabla}$ is the restriction of the Levi-Civita connection of $\mathbb{A d S}^{3}$ to $\sigma(\tilde{S})$. It can be easily shown that $\widetilde{B}$ is a self-adjoint operator of $T \widetilde{S}$. The second fundamental form of $\sigma$ is the symmetric two-tensor defined by $\widetilde{I}(\widetilde{v}, \widetilde{w})=\langle\widetilde{B} \widetilde{v}, \widetilde{w}\rangle$, whereas the third fundamental form is the symmetric tensor defined by $\widetilde{I I}(\widetilde{v}, \widetilde{w})=\langle\widetilde{B} \widetilde{v}, \widetilde{B} \widetilde{w}\rangle$. The proof of the following lemma is in Section 2.4.

Lemma 1.9 Let $h, h^{\star}$ be a pair of normalized metrics, let $\theta \in(0, \pi)$. There exists a unique equivariant embedding $(\phi, \rho)$ of $\widetilde{S}$ in $\mathbb{A d}^{3}$ with induced metric $\cos ^{2}(\theta / 2) \tilde{h}$ and third fundamental form $\sin ^{2}(\theta / 2) \tilde{h}^{\star}$. Moreover, $\rho$ is the holonomy representation of an MGHC AdS manifold $N$ : the first factor in $L_{e^{i \theta}}\left(h, h^{\star}\right)$ is the left representation of $N$ and the first factor in $L_{e^{-i \theta}}\left(h, h^{\star}\right)$ is the right representation of $N$.

\subsection{The center of circles in Teichmüller space}

Let $h, h^{\star} \in \mathcal{T}$. For each $\theta \in \mathbb{R}$, let $\left(h_{\theta}, h_{\theta}^{\star}\right)=L_{e^{i \theta}}\left(h, h^{\star}\right)$. According to Theorem 1.3, there is a unique minimal Lagrangian diffeomorphism $m_{\theta}:\left(S, h_{\theta}\right) \rightarrow\left(S, h_{\theta}^{\star}\right)$ isotopic to the identity. We can then consider on $S$ the conformal structure $c_{\theta}$ of the metric $h_{\theta}+m_{\theta}^{*} h_{\theta}^{\star}$. We call $c_{\theta}$ the center of $\left(h_{\theta}, h_{\theta}^{\star}\right)$. This conformal class of metrics has some interesting properties, proved in Section 3.4.

Theorem $1.10 \quad$ (i) $m_{\theta}: S \rightarrow S$ is the identity map — that is, the identity is minimal Lagrangian between $\left(S, h_{\theta}\right)$ and $\left(S, h_{\theta}^{\star}\right)$.

(ii) $c_{\theta}$ does not depend on $\theta$, it is equal to a fixed conformal class $c$.

(iii) Let $f_{\theta}:(S, c) \rightarrow\left(S, h_{\theta}\right)$ and $f_{\theta}^{\star}:(S, c) \rightarrow\left(S, h_{\theta}^{\star}\right)$ be the unique harmonic maps isotopic to the identity. Then $f_{\theta}$ and $f_{\theta}^{\star}$ have opposite Hopf differentials.

(iv) $c$ is the unique minimum of the functional

$$
E(\cdot, h)+E\left(\cdot, h^{\star}\right): \mathcal{T} \rightarrow \mathbb{R},
$$

where $E\left(c^{\prime}, h\right)$ is the energy of the unique harmonic map $\left(S, c^{\prime}\right) \rightarrow(S, h)$ isotopic to the identity.

(v) For any $\theta \in \mathbb{R}$,

$$
\Phi\left(f_{\theta}\right)=e^{i \theta} \Phi\left(f_{0}\right) .
$$




\subsection{Obtaining $R$ from $L$}

As a consequence of Theorem 1.10, we find a simple relation between the map $R$ defined earlier in terms of Hopf differential, and the map $L$.

Corollary 1.11 Let $\left(h, h^{\star}\right)$ be a couple of normalized metrics and let $c$ be the conformal class of $h+h^{\star}$. For any $e^{i \theta} \in S^{1}$, we have

$$
L_{e^{i \theta}}\left(h, h^{\star}\right)=\left(R_{c, \theta}(h), R_{c, \theta+\pi}\left(h^{\star}\right)\right) .
$$

\subsection{The earthquake flow as a limit}

Theorem 1.12 Let $h \in \mathcal{T}$, let $\left(h_{n}^{\star}\right)_{n \in \mathbb{N}}$ be a sequence of hyperbolic metrics and let $\lambda \neq 0$ be a measured lamination. Consider a sequence $\left(\theta_{n}\right)_{n \in \mathbb{N}}$ of positive real numbers such that $\lim _{n \rightarrow \infty} \theta_{n} \ell_{h_{n}^{\star}}=\iota(\lambda, \bullet)$ in the sense of convergence of the length spectra of simple closed curves. Then

$$
\lim _{n \rightarrow+\infty} h_{n}^{1}=E_{\lambda / 2}(h), \quad \lim _{n \rightarrow+\infty} \theta_{n} \ell_{h_{n}^{2}}=\iota(\lambda, \bullet),
$$

where $\left(h_{n}^{1}, h_{n}^{2}\right):=L_{e^{i \theta_{n}}}\left(h, h_{n}^{\star}\right)$.

At first sight it would appear more natural to take the limit where the sequence of centers $\left(c_{n}\right)$ converges projectively to $\lambda$. However the statement obtained by replacing $L$ by $R$ and $h_{n}^{\star}$ by $c_{n}$ in Theorem 1.12 turns out to be false, as proved-in one example-in Section 7; see Corollary 7.3.

Remark 1.13 In [43], Wolf showed that if $c$ is fixed and $h$ converges to a point $[\lambda]$ on the boundary of Teichmüller space, then the horizontal foliation of the Hopf differential $\Phi$ of the harmonic map $f:(S, c) \rightarrow(S, h)$ projectively converges to [ $\lambda]$ (where we are canonically identifying measured foliations and measured laminations). Indeed, the harmonic map $f$ converges to the harmonic map from $(S, c)$ to the $\mathbb{R}$-tree dual to $\lambda$; see Wolf [45].

On the other hand, in [30] Minsky showed that if $h$ is fixed and $c$ goes to some point $[\lambda]$ on the boundary of Teichmüller space, then in general the horizontal foliation of the Hopf differential of the harmonic map $f:(S, c) \rightarrow(S, h)$ does not (projectively) converge to $[\lambda]$ (though the projective limit points of those horizontal foliations share the same support of $\lambda$ if $c$ moves along a Teichmüller geodesic).

The fact that the convergence result in Theorem 1.12 holds in contrast with results in [30] relies on the fact that $h^{\star}$ is a hyperbolic metric whereas $c$ is only a conformal class and Thurston boundary of Teichmüller space is related to the asymptotic behavior of the hyperbolic invariants of the surface (see [30] for a discussion on this point). 
The heuristic argument motivating Theorem 1.12 involves the convergence of constant Gauss curvature surfaces to a pleated surface in $\mathbb{A d S}^{3}$. However, writing a proof based on these ideas turns out to be more difficult than it appears. A key technical statement is that minimal Lagrangian maps have a close proximity to Thurston compactification of Teichmüller space: minimal Lagrangian maps isotopic to the identity provide a correct normalization to understand the convergence of a sequence of hyperbolic metrics to a projective measured lamination in Thurston boundary of $\mathcal{T}$, in the following sense.

Theorem 1.14 Let $h$ be a hyperbolic metric on $S$, and let $\left(h_{n}^{\star}\right)_{n \in \mathbb{N}}$ be a sequence of hyperbolic metrics such that $\theta_{n} \ell_{h_{n}^{\star}} \rightarrow \iota(\lambda, \bullet)$, where $\lambda$ is a measured geodesic lamination, the $\theta_{n}$ are positive numbers, and the convergence is in the sense of the length spectrum. For each $n$, let $m_{n}:(S, h) \rightarrow\left(S, h_{n}^{\star}\right)$ be the minimal Lagrangian diffeomorphism isotopic to the identity. Then, for every smooth arc $\alpha$ in $S$ that meets the $h$-geodesic representative of $\lambda$ transversely and with endpoints not in the support of (the $h$-geodesic representative of) $\lambda$, the length for $\theta_{n}^{2} m_{n}^{*}\left(h_{n}^{\star}\right)$ of the geodesic segment homotopic to $\alpha$ (with fixed endpoints) converges to the intersection between $\alpha$ and $\lambda$.

The proof of this theorem involves the convergence of smooth surfaces to a pleated limit, but in the hyperbolic, rather than the anti-de Sitter, context.

\subsection{An extension of the Earthquake Theorem}

We can now state an extension to the landslide flow $L$ of Thurston's Earthquake Theorem (see [20]). Recall that this theorem states that, given two hyperbolic metrics $h$ and $h^{\prime}$ on a surface, there is a unique measured lamination $\lambda$ such that the left earthquake along $\lambda$ sends $h$ to $h^{\prime}$.

Theorem 1.15 Let $h, h^{\prime} \in \mathcal{T}$ and let $e^{i \theta} \in S^{1} \backslash\{0\}$. There is a unique $h^{\star} \in \mathcal{T}$ such that $L_{e^{i \theta}}^{1}\left(h, h^{\star}\right)=h^{\prime}$.

We give in Section 4.2 a simple proof based on a recent result of Barbot, Béguin and Zeghib [4] on the existence and uniqueness of constant Gauss curvature foliations in MGHC AdS manifolds.

As an easy consequence, a similar statement holds also for the flow $R$.

\subsection{A complex extension}

The earthquake flow has an extension as a map $E: \overline{\mathbb{H}} \times \mathcal{T} \times \mathcal{M L} \rightarrow \mathcal{T}$, where $\overline{\mathbb{H}}$ is the set of complex numbers with nonnegative imaginary part. This map has the property 
that, for any $h \in \mathcal{T}$ and any $\lambda \in \mathcal{M L}$, the map $z \mapsto E(z, h, \lambda)$ is holomorphic; see [28]. It can be defined in terms of grafting, or (for small $\lambda$ ) in terms of pleated surfaces in hyperbolic 3-space.

In Section 5 we prove that the landslide map $L$ defined above has a similar holomorphic extension where the parameter $e^{i \theta}$ is replaced by a complex number $\zeta$ in the closed unit disk. This defines many holomorphic disks in Teichmüller space; see Theorem 5.1. Similarly to what happens for complex earthquakes, this construction factors through the space of complex projective structures on $S$ for $\zeta \neq 0$, and the complex cyclic flow provides punctured holomorphic disks in this space. This factorization however does not extend for $\zeta=0$.

The complex landslide map limits to complex earthquakes just as the "real" landslide flow limits to the earthquake flow; see Theorem 6.1.

We hope at some point in the future to give another proof of the holomorphicity of this complex landslide map, based on a geometric argument taking place in the complexification of $\mathbb{H}^{3}$. This line of argument should also provide a straightforward and geometric way to understand why complex earthquakes are holomorphic disks.

\subsection{Landslide on the universal Teichmüller space}

Recall that a homeomorphism of the circle is quasisymmetric if and only if it is the boundary value of a quasiconformal diffeomorphism of the disk.

Definition 1.16 The universal Teichmüller space $\mathcal{T}_{U}$ is the quotient of the group $\mathcal{Q S}$ of quasisymmetric homeomorphisms of the circle by left composition by projective transformations.

The universal Teichmüller space contains embedded copies of the Teichmüller space of all closed surfaces. Indeed, consider a closed surface $S$ of genus at least 2, a fixed hyperbolic metric $h^{\#}$ on $S$ and its holonomy representation $\rho^{\#}: \pi_{1}(S) \rightarrow \operatorname{PSL}_{2}(\mathbb{R})$. Given a hyperbolic metric $h$ on $S$ and its holonomy representation $\rho: \pi_{1}(S) \rightarrow \operatorname{PSL}_{2}(\mathbb{R})$, there is a quasiconformal map $\tilde{f}: \mathbb{H}^{2} \rightarrow \mathbb{H}^{2}$ conjugating $\rho^{\#}$ and $\rho$. Moreover, the boundary value $\partial \tilde{f}: \partial_{\infty} \mathbb{H}^{2} \rightarrow \partial_{\infty} \mathbb{H}^{2}$ is uniquely determined by $\rho^{\#}$ and $\rho$, and the map sending $h$ to $\partial \tilde{f}$ is an embedding of $\mathcal{T}_{S}$ in $\mathcal{T}_{U}$; see eg Gardiner and Harvey [14].

Let $\psi: S^{1} \rightarrow S^{1}$ be a quasisymmetric homeomorphism. There is (see the first and third authors [9]) a unique minimal Lagrangian quasiconformal diffeomorphism $m: \mathbb{H}^{2} \rightarrow \mathbb{H}^{2}$ with $\partial m=\psi$. As for closed surfaces, there is then a unique bundle morphism $b: T \mathbb{H}^{2} \rightarrow T \mathbb{H}^{2}$ such that 
- $b$ is self-adjoint,

- it satisfies the Codazzi equation $d^{\nabla} b=0$, where $\nabla$ is the Levi-Civita connection for the hyperbolic metric,

- $m^{*} g=g(b \bullet, b \bullet)$, where $g$ is the hyperbolic metric on $\mathbb{H}^{2}$.

For every $\theta \in \mathbb{R}$ we then consider $\beta_{\theta}:=\cos (\theta / 2) E+\sin (\theta / 2) b$, where $E$ is the identity and $g_{\theta}:=g\left(\beta_{\theta} \bullet, \beta_{\theta} \bullet\right)$.

Lemma 1.17 $g_{\theta}$ is a complete hyperbolic metric on $\mathbb{H}^{2}$. The identity map between $\left(\mathbb{H}^{2}, g\right)$ and $\left(\mathbb{H}^{2}, g_{\theta}\right)$ is quasiconformal (and minimal Lagrangian), and its extension $\psi_{\theta}: S^{1} \rightarrow S^{1}$ to the boundary of $\mathbb{H}^{2}$ is quasisymmetric, so that it defines a point in $\mathcal{Q S}$.

In Section 8 we show how to use this fact to construct an extension of $L$ to a nontrivial circle action $\mathcal{L}$ on $\mathcal{T}_{U} \times \mathcal{T}_{U}$ (see Theorem 8.5).

\subsection{Content of the paper}

In Section 2 we present the background material, concerning in particular the relation between minimal Lagrangian diffeomorphisms of hyperbolic surfaces and globally hyperbolic AdS manifolds. In Section 3 we define the landslide flow and prove that it is indeed a flow (Theorem 1.8) as well as Theorem 1.10. In Section 4 we give the proof of the extension to the landslide flow of Thurston's Earthquake Theorem (Theorem 1.15). Then in Section 5 we construct the complex landslide map, actually as a map from $\mathcal{T} \times \mathcal{T} \times \dot{\bar{\Delta}}$ to $\mathcal{P}$, where $\dot{\bar{\Delta}}$ is the pointed closed unit disk in $\mathbb{C}$, and we prove that it is holomorphic and that it extends over $\bar{\Delta}$ as a map to $\mathcal{T}$. Section 6 considers the limit when the parameter $h^{\star}$ converges projectively to a measured lamination at Thurston boundary of Teichmüller space, and contains the proof of Theorem 1.12 as well as its complex extension, Theorem 6.1. In Section 7, on the other hand, we show that the situation is not as simple for the "center" $c$ determined by a fixed metric $h$ and a sequence $h_{n}^{\star}$ going to a point at infinity in Thurston compactification of $\mathcal{T}$ : the limit of the corresponding sequence of centers does not depend only on the limit of $\left(h_{n}^{\star}\right)$. Section 8 deals with the circle action on the universal Teichmüller space, while Section 9 contains some remarks and open questions.

Acknowledgments We are grateful to the Institute of Mathematical Sciences of the National University of Singapore, where most of the results presented here were obtained. The second named author would like to thank Mike Wolf for helpful clarifications on his paper [44]. 
We would also like to thank an anonymous referee for many remarks and suggestions that improved the readability of the paper.

FB is partially supported by the ANR through project Geodycos. J-M S was partially supported by the ANR through projects ETTT, ANR-09-BLAN-0116-01, and GeomEinstein, ANR-09-BLAN-0116-01. F B and G M are partially supported by FIRB project Geometria e topologia delle varietà in bassa dimensione (code RBFR10GHHH).

\section{Minimal lagrangian maps and AdS geometry}

We present in this section some background material used in the paper.

\subsection{Notation}

In this paper we consider a closed, oriented surface $S$ of genus at least 2 .

We consider $\mathbb{A d S}^{3}$, as well as all AdS manifolds, as oriented and time-oriented. All the embeddings of $S$ that we consider will implicitly be considered as time-oriented, that is, the oriented normal to the image is future-oriented. Moreover, the convex embeddings will always be considered to be positively convex, that is, the oriented normal is futuredirected and pointing towards the convex side. We recall that, by Mess' work [29], it is possible to identify the isometries of $\mathbb{A d S}^{3}$ with double cover of $\mathrm{PSL}_{2}(\mathbb{R}) \times \mathrm{PSL}_{2}(\mathbb{R})$ in such a way that, if $S$ is a positively convex pleated surface in $\mathbb{A d S}^{3}$, bent along $\lambda$ and with first fundamental form $h$, then the first (resp. second) factor corresponds to the holonomy of the hyperbolic surface obtained from $h$ performing a left (resp. right) earthquake along $\lambda$.

\subsection{Hyperbolic ends}

The 3-dimensional hyperbolic space can be defined as a quadric in the 4-dimensional Minkowski space $\mathbb{R}^{1,3}=\left(\mathbb{R}^{4},-d x_{0}^{2}+d x_{1}^{2}+d x_{2}^{2}+d x_{3}^{2}\right)$, with the induced metric

$$
\mathbb{H}^{3}=\left\{x \in \mathbb{R}^{1,3} \mid\langle x, x\rangle=-1 \text { and } x_{0}>0\right\} .
$$

It is a simply connected, complete manifold with constant curvature -1 .

A quasi-Fuchsian hyperbolic manifold is a 3-dimensional manifold locally isometric to $\mathbb{H}^{3}$, homeomorphic to $S \times \mathbb{R}$, which contains a nonempty compact convex subset.

Such a quasi-Fuchsian manifold $M$ contains a smallest nonempty convex subset $C(M)$ called its convex core. $M$ is Fuchsian if $C(M)$ is a totally geodesic surface, otherwise the boundary of $C(M)$ is the disjoint union of two pleated surfaces. 
Each connected component of the complement of $C(M)$ in $M$ is an instance of a hyperbolic end: a hyperbolic manifold homeomorphic to $S \times \mathbb{R}_{>0}$, complete on one side and bounded by a locally concave pleated surface on the other. There is a one-toone correspondence between hyperbolic ends homeomorphic to $S \times \mathbb{R}_{>0}$ and complex projective structures on $S$, which associates to a hyperbolic end the natural complex projective structure on its boundary at infinity; see eg [23].

Labourie [25] proved that any hyperbolic end has a unique foliation by convex, constant curvature surfaces. The curvature varies monotonically from -1 close to the pleated surface boundary, to 0 close to the boundary at infinity.

Given an oriented surface $\Sigma$ in a hyperbolic end $M$ (or in $\mathbb{H}^{3}$ ) we will usually denote by $I$ its induced metric, and by $B$ its shape operator, considered as a bundle morphism from $T \Sigma$ to $T \Sigma$. It is defined by $B X=\nabla_{X} v$, where $v$ is the oriented unit normal to $\Sigma$ and $\nabla$ is the Levi-Civita connection of $M$. We will also denote by $E: T S \rightarrow T S$ the identity.

Definition 2.1 Let $\Sigma$ be a convex surface embedded in a hyperbolic end $M$ with embedding data $\left(I_{\Sigma}, B_{\Sigma}\right)$. The grafted metric on $\Sigma$ is $I_{\Sigma}^{\#}=I_{\Sigma}\left(\left(E+B_{\Sigma}\right) \bullet,\left(E+B_{\Sigma}\right) \bullet\right)$.

A basic and well-known property of this metric $I_{\Sigma}^{\#}$ is that the hyperbolic Gauss mapsending a point $x \in \Sigma$ to the endpoint at infinity of the geodesic ray starting at $x$ orthogonal to $\Sigma$-is a conformal map between $\left(\Sigma, I_{\Sigma}^{\#}\right)$ and $\partial_{\infty} M$ with its conformal structure. More details will be found in Section 6.

\subsection{The duality between hyperbolic and de Sitter ends}

The 3-dimensional de Sitter space can be defined, as the hyperbolic space, as a quadric in the 4-dimensional Minkowski space, with the induced metric

$$
\mathrm{d} \mathbb{S}^{3}=\left\{x \in \mathbb{R}^{1,3} \mid\langle x, x\rangle=1\right\} .
$$

There is a one-to-one correspondence between points in $\mathrm{dS}^{3}$ and oriented totally geodesic planes in $\mathbb{H}^{3}$; see eg Hodgson and Rivin [17] and the third author [36]. Given an oriented surface $S \subset \mathbb{H}^{3}$, its dual is the set $S^{\star}$ of points of $\mathrm{dS}^{3}$ corresponding to oriented planes tangent to $S$ in $\mathbb{H}^{3}$. If $S$ is smooth and locally strictly convex, then $S^{\star}$ is also smooth and locally strictly convex.

Consider a quasi-Fuchsian hyperbolic manifold $M$, and let $E$ be one of the ends of $M$, that is, one of the connected components of the complement of $C(M)$ in $M$. The universal cover of $M$ is identified with $\mathbb{H}^{3}$, and the universal cover $\widetilde{E}$ of $E$ is then identified with a connected component of the complement of the convex hull of the 
limit set $\Lambda$ of $\pi_{1}(M)$ in $\partial_{\infty} \mathbb{H}^{3}$. Let $\widetilde{E}^{\star}$ be the set of points of $\mathrm{dS}^{3}$ corresponding to oriented planes in $\mathbb{H}^{3}$ contained in $\widetilde{E}$. Then (see [29]) $\pi_{1}(M)$ acts properly discontinuously on $\widetilde{E}^{\star}$, and the quotient is a de Sitter domain of dependence, that is, an MGHC de Sitter manifold (see Section 1.7 for the definition in the AdS case).

This construction actually extends (see [29]) to a hyperbolic end $E$ which is not necessarily one of the ends of a quasi-Fuchsian manifold. In this manner, any hyperbolic end $E$ has a "dual" de Sitter domain of dependence $E^{\star}$, and conversely.

One feature of this duality which will be used below is that if $S$ is a surface in $E$ with constant curvature $K$, then there is a dual surface $S^{\star}$ in $E^{\star}$. (It is the quotient by $\pi_{1}(M)$ of the surface in $\mathrm{dS}^{3}$ dual to the universal cover of $S$ in $\mathbb{H}^{3}$.) The curvature of $S^{\star}$ is then also constant, and equal to $K /(K+1)$. In this manner a foliation of $E$ by constant curvature surfaces gives rise to a dual foliation of $E^{\star}$ by constant curvature surfaces (see [4] for more details).

\subsection{Globally hyperbolic AdS manifolds}

The definition of $\mathbb{A d S}^{3}$ and of globally hyperbolic AdS manifolds are recalled in the introduction. There are many similarities between quasi-Fuchsian hyperbolic manifolds and MGHC AdS manifolds, some of which—being used in the arguments below-are recalled here.

Let $N$ be an MGHC AdS 3-manifold, and let $F$ be a closed, space-like surface in $N$ for which the induced metric has negative sectional curvature (or, equivalently, the determinant of the second fundamental form of $F$ is everywhere larger than -1 ). Let $I$ and $v$ be the induced metric on this surface and a unit normal vector. Let $J$ be the complex structure induced by $v$ on $F$ : namely $J(v)=v \times v$ where $\times$ is the vector product on $T \mathbb{A d S}^{3}$. Finally, let $B=\nabla v$ be the shape operator of $F$, where $\nabla$ is the Levi-Civita connection of $\mathbb{A d S}^{3}$. We consider the Riemannian metrics on $F$

$$
h_{l}=I((E+J B) \bullet,(E+J B) \bullet), \quad h_{r}=I((E-J B) \bullet,(E-J B) \bullet) .
$$

Then $h_{l}$ and $h_{r}$ are two smooth hyperbolic metrics on $F$ (see Krasnov and the third author [21]). This can be used when $F$ is a maximal or constant mean curvature surface in $N$, but also when $F$ is a constant Gauss curvature surface.

Remark 2.2 Notice that, even if $J$ and $B$ depend on the choice of a normal vector, $J B$ and the metrics $h_{l}$ and $h_{r}$ are independent of it.

According to our orientation and time-orientation of $\mathbb{A d S}^{3}$, the holonomy of the metric $h_{l}$ is equal to the first factor of the holonomy of $N$ and the holonomy of $h_{r}$ is equal to the second factor of the holonomy of $N$; see [21]. This last observation can be used to prove Lemma 1.9 . 
Proof of Lemma 1.9 Let $b: T S \rightarrow T S$ be the positive $h$-self-adjoint operator given by Corollary 1.5 such that $h^{\star}=h(b \bullet, b \bullet)$. Since $h$ and $h^{\star}$ are normalized metrics we know that $b$ is a solution of Codazzi equation and $\operatorname{det} b=1$.

We consider now the pair

$$
I_{\theta}=\cos ^{2}(\theta / 2) h, \quad B_{\theta}=\tan (\theta / 2) b .
$$

Clearly, $B_{\theta}$ is a solution of Codazzi equation. Moreover, since $I_{\theta}$ is a metric of constant curvature $K=-\frac{1}{\cos ^{2}(\theta / 2)}$, we easily get that

$$
K_{\theta}=-1-\operatorname{det} B_{\theta},
$$

so $\left(I_{\theta}, B_{\theta}\right)$ is also a solution of Gauss equation for spacelike AdS surfaces (see [21]). This implies (see Tenenblat [41] and Jacobowitz [18]) that there is an equivariant map

$$
\phi: \widetilde{S} \rightarrow \widetilde{F} \subset \mathbb{A d}^{3},
$$

whose embedding data are $\widetilde{I}_{\theta}$ and $\widetilde{B}_{\theta}$. The map $\phi$ is unique up to isometries of $\mathbb{A d S}^{3}$. We will also require that

- the normal field $\widetilde{v}$ that induces the right orientation on $\widetilde{S}$ points toward the convex side,

- $\tilde{v}$ is a future-directed vector field.

Since $\phi$ induces on $\widetilde{S}$ a complete metric, $\phi$ is an embedding and the components of the holonomy $\rho_{l}, \rho_{r}: \pi_{1}(S) \rightarrow \mathrm{PSL}_{2}(\mathbb{R})$ are Fuchsian representations (see [29]).

On the other hand by [21] the hyperbolic surfaces $\mathbb{H}^{2} / \rho_{l}$ and $\mathbb{H}^{2} / \rho_{r}$ are isometric to the Riemann surfaces $\left(S, h_{l}\right)$ and $\left(S, h_{r}\right)$.

Lemma 1.9 and [21] imply that $L_{e^{i \theta}}\left(h, h^{\star}\right)$ is a couple of hyperbolic metrics. For convenience of the reader, we will give a more direct proof of this fact in Section 3.1.

MGHC AdS manifolds have a unique foliation by constant mean curvature surfaces; see Barbot, Béguin and Zeghib [3].

An MGHC AdS manifold $N$ contains a smallest nonempty convex subset $C(N)$, called its convex core: $N$ is called Fuchsian if $C(N)$ is a totally geodesic surface; otherwise, the boundary of $C(N)$ is the disjoint union of two pleated locally convex surfaces in $N$, so that its induced metric is hyperbolic and its pleating is described by a measured lamination (see [29]). The complement of $C(N)$ in $N$ has two connected components, one future convex, the other past convex. Barbot, Béguin and Zeghib [4] proved that $N \backslash C(N)$ has a unique foliation by convex, constant Gauss curvature surfaces. The curvature is monotonic along the foliation, and varies from -1 in the neighborhood of the convex core to $-\infty$ near the initial/final singularity. 


\subsection{Minimal Lagrangian maps between hyperbolic surfaces}

The definition of minimal Lagrangian diffeomorphisms has been recalled in the introduction. Remark that the definition directly shows that the inverse of a minimal Lagrangian diffeomorphism is also minimal Lagrangian.

Let us mention here that they occur in several distinct geometric contexts, and the interplay between the different occurrences is used below, in particular in Section 4.

- If $S$ is a surface of constant curvature $K$ in a constant curvature, Riemannian or Lorentzian 3-manifold $M$, then the third fundamental form III of $S$ also has constant curvature $K^{\star}$, where $K^{\star}$ depends on $K$, on the ambient curvature, and on whether the ambient space is Riemannian or Lorentzian (for instance, if $M=\mathbb{H}^{3}$, then $\left.K^{\star}=K /(K+1)\right)$. If both $K$ and $K^{\star}$ are negative, then $|K| I$ and $\left|K^{\star}\right| I I I$ are hyperbolic metrics, and the identity, considered as a map from $(S,|K| I)$ to $\left(S,\left|K^{\star}\right| I I I\right)$, is minimal Lagrangian.

- If $M$ is an "almost Fuchsian" manifold, that is, $M$ is a quasi-Fuchsian hyperbolic 3-manifolds containing a closed, embedded minimal surface $S$ with principal curvatures everywhere in $(-1,1)$, then $S$ is the unique closed minimal surface in $M$. The "hyperbolic Gauss maps" send $S$ to each connected component of the boundary at infinity of $M$, and both maps are diffeomorphisms. Composing these maps one finds a diffeomorphism between one component of $\partial_{\infty} M$ and the other. This diffeomorphism is minimal Lagrangian if each boundary component is endowed with the (unique) hyperbolic metric in its conformal class. (See eg [21] for details and proofs.)

- Similarly, if $N$ is an MGHC AdS manifold, then it contains a unique closed, space-like maximal surface $F$. Consider the metrics $h_{l}$ and $h_{r}$ defined above on $F$. Then $h_{l}$ and $h_{r}$ are the left and right hyperbolic metrics of $N$, respectively, and moreover the identity between $\left(F, h_{l}\right)$ and $\left(F, h_{r}\right)$ is minimal Lagrangian (see [21] for details).

It is the first of these occurrences which will play the largest role here.

Minimal Lagrangian maps between hyperbolic surfaces are intimately related to harmonic maps: let $m:(S, h) \rightarrow\left(S^{\prime}, h^{\prime}\right)$ be a minimal Lagrangian diffeomorphism between two hyperbolic surfaces and consider the conformal structure $c$ on $S$ of the metric $h+m^{*} h^{\prime}$. Then

- the identity is harmonic between $(S, c)$ and $(S, h)$,

- $m$ is harmonic between $(S, c)$ and $\left(S^{\prime}, h^{\prime}\right)$,

- those two harmonic maps have opposite Hopf differentials.

The converse is also true. Details can be found eg in [38]. 


\section{Definition of the cyclic flow}

In this section we consider two fixed normalized hyperbolic metrics $h, h^{\star}$ on $S$, and call $b$ the bundle morphism given by Corollary 1.5. Let $\beta_{\theta}$ be the family of operators defined in Equation (1) in Section 1.6.

Definition 3.1 Given $\theta \in \mathbb{R}$, we call

$$
h_{\theta}=h\left(\beta_{\theta} \bullet, \beta_{\theta} \bullet\right) \text {. }
$$

Comparing with Definition 1.6, we have $L_{e^{i \theta}}\left(h, h^{\star}\right)=\left(h_{\theta}, h_{\theta+\pi}\right)$.

\subsection{The Levi-Civita connection of $h_{\theta}$}

Lemma 3.2 For all $\theta \in \mathbb{R}, d^{\nabla} \beta_{\theta}=0$, where $\nabla$ is the Levi-Civita connection of $h$.

Proof Let $u, v$ be two vector fields on $S$. Note first that $\beta_{\theta}$ satisfies the Codazzi equation

$$
\begin{aligned}
\left(d^{\nabla} \beta_{\theta}\right)(u, v)= & \nabla_{u}\left(\beta_{\theta} v\right)-\nabla_{v}\left(\beta_{\theta} u\right)-\beta_{\theta}([u, v]) \\
=\cos (\theta / 2)\left(\nabla_{u} v-\nabla_{v} u-\right. & {[u, v]) } \\
& +\sin (\theta / 2)\left(\nabla_{u}(J b v)-\nabla_{v}(J b u)-J b[u, v]\right) .
\end{aligned}
$$

Since $J$ is parallel for $\nabla$, we have that $\nabla J=J \nabla$, so

$$
\left(d^{\nabla} \beta_{\theta}\right)(u, v)=\sin (\theta / 2) J\left(\nabla_{u}(b v)-\nabla_{v}(b u)-b[u, v]\right)=0,
$$

where the last equality follows from the fact that $b$ satisfies Codazzi equation.

Lemma 3.3 The Levi-Civita connection $\nabla^{\theta}$ of $h_{\theta}$ is given by

$$
\nabla_{u}^{\theta} v=\beta_{-\theta} \nabla_{u}\left(\beta_{\theta} v\right)
$$

Proof Consider the connection $\nabla^{\theta}$ defined in the statement of the lemma. It is sufficient to prove that it is compatible with $h_{\theta}$ and torsion-free.

Let $u, v, w$ be three vector fields on $S$. Then

$$
\begin{aligned}
u \cdot h_{\theta}(v, w) & =u \cdot h\left(\beta_{\theta} v, \beta_{\theta} w\right) \\
& =h\left(\nabla_{u}\left(\beta_{\theta} v\right), \beta_{\theta} w\right)+h\left(\beta_{\theta} v, \nabla_{u}\left(\beta_{\theta} w\right)\right) \\
& =h_{\theta}\left(\nabla_{u}^{\theta} v, w\right)+h_{\theta}\left(v, \nabla_{u}^{\theta} w\right)
\end{aligned}
$$

and therefore $\nabla^{\theta}$ is compatible with $h_{\theta}$. 
We can now compute the torsion of $\nabla^{\theta}$ on $u, v$ :

$$
\begin{aligned}
\nabla_{u}^{\theta} v-\nabla_{v}^{\theta} u-[u, v] & =\beta_{-\theta}\left(\nabla_{u}\left(\beta_{\theta} v\right)-\nabla_{v}\left(\beta_{\theta} u\right)-\beta_{\theta}[u, v]\right) \\
& =\beta_{-\theta}\left(d^{\nabla} \beta_{\theta}\right)(u, v) \\
& =0 .
\end{aligned}
$$

Thus $\nabla^{\theta}$ is torsion-free and compatible with $h_{\theta}$, and so it is the Levi-Civita connection of $h_{\theta}$.

The computation of the connection of $h_{\theta}$ allows to prove easily that $h_{\theta}$ is a hyperbolic metric.

Proof of Proposition 1.7 Let $\left(e_{1}, e_{2}\right)$ be an orthonormal frame for $h$ on $S$, with connection 1 -form $\omega$ with respect to the Levi-Civita connection $\nabla$ of $h$. Let $\left(e_{1}^{\prime}, e_{2}^{\prime}\right)=\left(\beta_{\theta}^{-1} e_{1}, \beta_{\theta}^{-1} e_{2}\right)$, it is an orthonormal moving frame for $h_{\theta}$. Since the Levi-Civita connection $\nabla^{\theta}$ of $h_{\theta}$ is given by Lemma 3.3, a direct computation shows that the connection 1 -form of $\left(e_{1}^{\prime}, e_{2}^{\prime}\right)$ for $\nabla^{\theta}$ is also equal to $\omega$. Since the identity map between $(S, h)$ and $\left(S, h_{\theta}\right)$ is area-preserving, it follows that $h$ and $h_{\theta}$ have the same curvature, so that $h_{\theta}$ is also hyperbolic.

\subsection{A cyclic property}

Our next goal is to prove Theorems 1.8 and 1.10. The proofs are in the next sections, after some preliminary lemmas.

Lemma 3.4 Let $\theta, \theta^{\prime} \in \mathbb{R}$. Then $\beta_{\theta} \circ \beta_{\theta^{\prime}}=\beta_{\theta+\theta^{\prime}}$.

Proof By definition we have

$$
\begin{aligned}
\beta_{\theta} \circ \beta_{\theta^{\prime}}= & (\cos (\theta / 2) E+\sin (\theta / 2) J b) \circ\left(\cos \left(\theta^{\prime} / 2\right) E+\sin \left(\theta^{\prime} / 2\right) J b\right) \\
= & \cos (\theta / 2) \cos \left(\theta^{\prime} / 2\right) E+\sin (\theta / 2) \sin \left(\theta^{\prime} / 2\right) J b J b+\left(\cos (\theta / 2) \sin \left(\theta^{\prime} / 2\right)\right. \\
& \left.+\sin (\theta / 2) \cos \left(\theta^{\prime} / 2\right)\right) J b .
\end{aligned}
$$

But $J b J b=-E$ because $b$ is self-adjoint and of determinant 1. It follows that

$$
\beta_{\theta} \circ \beta_{\theta^{\prime}}=\cos \left(\left(\theta+\theta^{\prime}\right) / 2\right) E+\sin \left(\left(\theta+\theta^{\prime}\right) / 2\right) J b=\beta_{\theta+\theta^{\prime}} .
$$

For instance, since $J b=\beta_{\pi}$, it follows that $J b \circ \beta_{\theta}=\beta_{\theta} \circ J b=\beta_{\theta+\pi}$.

Lemma 3.5 Let $b_{\theta}: T S \rightarrow T S$ be the bundle morphism associated to $h_{\theta}$ and $h_{\theta}^{\star}$ by Corollary 1.5. Then

$$
b_{\theta}=\beta_{-\theta} \circ b \circ \beta_{\theta} .
$$


Proof Let $b_{\theta}=\beta_{-\theta} \circ b \circ \beta_{\theta}$. This lemma will follow if we prove the following facts:

(i) $h_{\theta}\left(b_{\theta} \bullet, b_{\theta} \bullet\right.$ ) is isotopic to $h_{\theta}^{\star}$ (we will actually prove that it is equal to $h_{\theta}^{\star}$ );

(ii) $b_{\theta}$ has determinant 1 ;

(iii) $b_{\theta}$ is self-adjoint for $h_{\theta}$;

(iv) $d^{\nabla^{\theta}} \beta_{\theta}=0$.

For the first point, note that $J b=\beta_{\pi}$, and therefore

$$
\begin{aligned}
h_{\theta}\left(b_{\theta} \bullet, b_{\theta} \bullet\right) & =h\left(b \beta_{\theta} \bullet, b \beta_{\theta} \bullet\right)=h\left(J b \beta_{\theta} \bullet, J b \beta_{\theta} \bullet\right) \\
& =h\left(\beta_{\pi} \circ \beta_{\theta} \bullet, \beta_{\pi} \circ \beta_{\theta} \bullet\right)=h\left(\beta_{\pi+\theta} \bullet, \beta_{\pi+\theta} \bullet\right) \\
& =h_{\theta}^{\star} .
\end{aligned}
$$

The second point is clear since $\operatorname{det}\left(b_{\theta}\right)=\operatorname{det}\left(\beta_{-\theta}\right) \operatorname{det}(b) \operatorname{det}\left(\beta_{\theta}\right)=1$.

For point (iii) let $u, v$ be two vector fields on $S$, then

$$
\begin{aligned}
h_{\theta}\left(b_{\theta} u, v\right) & =h\left(\beta_{\theta} b_{\theta} u, \beta_{\theta} v\right)=h\left(b \beta_{\theta} u, \beta_{\theta} v\right) \\
& =h\left(\beta_{\theta} u, b \beta_{\theta} v\right)=h_{\theta}\left(u, b_{\theta} v\right) .
\end{aligned}
$$

For point (iv), let again be $u, v$ be two vector fields on $S$. Then, using the expression of $\nabla^{\theta}$ in Lemma 3.3, we have

$$
\begin{aligned}
\left(d^{\nabla^{\theta}} b_{\theta}\right)(u, v) & =\nabla_{u}^{\theta}\left(b_{\theta} v\right)-\nabla_{v}^{\theta}\left(b_{\theta} u\right)-b_{\theta}[u, v] \\
& =\beta_{-\theta} \nabla_{u}\left(\beta_{\theta} b_{\theta} v\right)-\beta_{-\theta} \nabla_{v}\left(\beta_{\theta} b_{\theta} u\right)-b_{\theta}[u, v] \\
& =\beta_{-\theta}\left(d^{\nabla} \beta_{\theta+\pi}\right)(u, v),
\end{aligned}
$$

so that $\left(d^{\nabla^{\theta}} b_{\theta}\right)(u, v)=0$ by Lemma 3.2. This completes the proof.

Remark 3.6 Lemma 3.5 shows that $h_{\theta}$ and $h_{\theta+\pi}$ are normalized metrics.

\subsection{Proof of Theorem 1.8}

Let $\theta, \theta^{\prime} \in \mathbb{R}$. By definition of $L_{\theta^{\prime}}$,

$$
L_{\theta^{\prime}}\left(L_{\theta}\left(h, h^{\star}\right)\right)=\left(h_{\theta}\left(\bar{\beta}_{\theta^{\prime}} \bullet, \bar{\beta}_{\theta^{\prime}} \bullet\right),\left(h_{\theta+\pi}\left(\bar{\beta}_{\theta^{\prime}} \bullet, \bar{\beta}_{\theta^{\prime}} \bullet\right)\right),\right.
$$

where

$$
\bar{\beta}_{\theta^{\prime}}=\cos \left(\theta^{\prime} / 2\right) E+\sin \left(\theta^{\prime} / 2\right) J_{\theta} b_{\theta},
$$


where $J_{\theta}$ is the complex structure of $h_{\theta}$. Clearly, $J_{\theta}=\beta_{-\theta} \circ J \circ \beta_{\theta}$, so that

$$
\begin{aligned}
\bar{\beta}_{\theta^{\prime}} & =\beta_{-\theta} \circ\left(\cos \left(\theta^{\prime} / 2\right) E+\sin \left(\theta^{\prime} / 2\right) J b\right) \circ \beta_{\theta} \\
& =\beta_{-\theta} \circ \circ \beta_{\theta^{\prime}} \circ \beta_{\theta} \\
& =\beta_{\theta^{\prime}} .
\end{aligned}
$$

We now see that

$$
\begin{aligned}
L_{\theta^{\prime}}\left(L_{\theta}\left(h, h^{\star}\right)\right) & =\left(h_{\theta}\left(\beta_{\theta^{\prime}} \bullet, \beta_{\theta^{\prime}} \bullet\right),\left(h_{\theta+\pi}\left(\beta_{\theta^{\prime}} \bullet, \beta_{\theta^{\prime}} \bullet\right)\right)\right. \\
& =\left(h\left(\beta_{\theta} \circ \beta_{\theta^{\prime}} \bullet, \beta_{\theta} \circ \beta_{\theta^{\prime}} \bullet\right), h\left(\beta_{\theta+\pi} \circ \beta_{\theta^{\prime}} \bullet, \beta_{\theta+\pi} \circ \beta_{\theta^{\prime}} \bullet\right)\right) \\
& =\left(h\left(\beta_{\theta^{\prime}+\theta} \bullet, \beta_{\theta^{\prime}+\theta} \bullet\right), h^{\star}\left(\beta_{\theta^{\prime}+\theta} \bullet, \beta_{\theta^{\prime}+\theta} \bullet\right)\right) \\
& =L_{\theta^{\prime}+\theta}\left(h, h^{\star}\right) .
\end{aligned}
$$

This proves Theorem 1.8.

\subsection{Proof of Theorem 1.10}

Point (i), namely the fact that $h_{\theta}$ and $h_{\theta+\pi}$ are normalized metrics, follows from Lemma 3.5.

We compute the expression of the metric $\widehat{c}_{\theta}=h_{\theta}+h_{\theta+\pi}$ which is in the conformal class of $c_{\theta}$ :

$$
\begin{aligned}
\widehat{c}_{\theta}= & h_{\theta}+h_{\theta+\pi} \\
= & h((\cos (\theta / 2) E+\sin (\theta / 2) b) \bullet,(\cos (\theta / 2) E+\sin (\theta / 2) b) \bullet) \\
& +h((-\sin (\theta / 2) E+\cos (\theta / 2) b) \bullet,(-\sin (\theta / 2) E+\cos (\theta / 2) b) \bullet) \\
= & \left(\cos ^{2}(\theta / 2)+\sin ^{2}(\theta / 2)\right) h+\left(\cos ^{2}(\theta / 2)+\sin ^{2}(\theta / 2)\right) h(b \bullet, b \bullet) \\
= & \widehat{c},
\end{aligned}
$$

so $c_{\theta}$ is indeed independent of $\theta$ : this proves (ii).

The fact that the identity $(S, c) \rightarrow\left(S, h_{\theta}\right)$ is harmonic follows from the last paragraph of Section 2.5.

Moreover, recall that $b_{\theta}$ has determinant 1 and that $h_{\theta}^{\star}=h_{\theta}\left(b_{\theta} \bullet, b_{\theta} \bullet\right)$. A simple computation then shows that $h_{\theta}-h_{\theta}^{\star}$ is traceless with respect to $h_{\theta}+h_{\theta}^{\star}$. The definition of Hopf differential (see Section 1.4) therefore shows that, if $\Phi_{\theta}$ is the Hopf differential of the identity from $(S, c)$ to $\left(S, h_{\theta}\right)$, then

$$
\frac{h_{\theta}-h_{\theta}^{\star}}{2}=2 \operatorname{Re}\left(\Phi_{\theta}\right) \text {. }
$$


Therefore

$$
\begin{aligned}
4 \operatorname{Re}\left(\Phi_{\theta}\right)= & h((\cos (\theta / 2) E+\sin (\theta / 2) b) \bullet,(\cos (\theta / 2) E+\sin (\theta / 2) b) \bullet) \\
& -h((-\sin (\theta / 2) E+\cos (\theta / 2) b) \bullet,(-\sin (\theta / 2) E+\cos (\theta / 2) b) \bullet) \\
= & \left(\cos ^{2}(\theta / 2)-\sin ^{2}(\theta / 2)\right)\left(h-h^{\star}\right)+4 \sin (\theta / 2) \cos (\theta / 2) h(b \bullet, \bullet) \\
= & \cos (\theta)\left(h-h^{\star}\right)+2 \sin (\theta) h(b \bullet, \bullet) .
\end{aligned}
$$

So $\operatorname{Re}\left(\Phi_{\theta}\right)=\operatorname{Re}\left(e^{i \theta} \Phi\right)$, where

$$
\Phi=\frac{h-h^{\star}+2 i h(b \bullet, \bullet)}{4}=\frac{h\left(\left(E-b^{2}\right) \bullet, \bullet\right)+2 i h(b \bullet, \bullet)}{4}
$$

is the Hopf differential of the identity from $(S, c)$ to $(S, h)$. This proves points (iii) and (v).

About point (iv), it was shown by Tromba [13] that $E(\bullet, h): \mathcal{T} \rightarrow \mathbb{R}$ is proper and so is $E(\bullet, h)+E\left(\bullet, h^{\star}\right)$. Moreover, by a result of Douglas [10] (see also Jost [19]) we have

$$
\left.d E(\bullet, h)\right|_{\bullet=c^{\prime}}=-\Psi,
$$

where $\Psi$ is the Hopf differential of the harmonic map $\left(S, c^{\prime}\right) \rightarrow(S, h)$ isotopic to the identity. Hence, $\left.\left(d E(\bullet, h)+d E\left(\bullet, h^{\star}\right)\right)\right|_{\bullet=c^{\prime}}=0$ if and only if the harmonic maps $\left(S, c^{\prime}\right) \rightarrow(S, h)$ and $\left(S, c^{\prime}\right) \rightarrow\left(S, h^{\star}\right)$ isotopic to the identity have opposite Hopf differentials, which exactly means that $c=c^{\prime}$ is the center of $\left(h, h^{\star}\right)$ (as follows from Remark 3.7).

\subsection{Centers}

We conclude this section by some remarks on the respective behavior of $h, h^{\star}$ and $c$. Let $\Phi$ be the Hopf differential of the unique harmonic map $f:(S, c) \rightarrow(S, h)$ homotopic to the identity (see Theorem 1.1).

Remark 3.7 The metric $h^{\star}$ is uniquely determined by $h$ and $c$. Moreover, there is a bijective correspondence between the couples $(c, \Phi)$ and the couples $\left(h, h^{\star}\right)$.

Proof Given $(c, \Phi)$, there exists a unique isotopy class of hyperbolic metric $h$ on $S$ and a unique harmonic map $f:(S, c) \rightarrow(S, h)$ isotopic to the identity with Hopf differential equal to $-\Phi$ (see [43]).

Hence, given $h$ and $c$, we determine $h^{\star}$ as the unique hyperbolic metric (up to isotopy) such that the harmonic map $f^{\star}:(S, c) \rightarrow\left(S, h^{\star}\right)$ has Hopf differential $-\Phi$. The content of Section 2.5 then indicates that $f^{\star} \circ f^{-1}$ is minimal Lagrangian, so that $c$ is the center of $\left(h, h^{\star}\right)$. 
The argument above also shows that $(c, \Phi)$ determines $\left(h, h^{\star}\right)$.

Conversely, given $\left(h, h^{\star}\right)$, we have seen that there is a unique minimal Lagrangian diffeomorphism $m:(S, h) \rightarrow\left(S, h^{\star}\right)$ isotopic to the identity. By definition, $c$ is the conformal class of $h+m^{*} h^{\star}$, the identity map $(S, c) \rightarrow(S, h)$ is harmonic with Hopf differential $\Phi$ and $m:(S, c) \rightarrow\left(S, h^{\star}\right)$ is harmonic with Hopf differential $-\Phi$. This determines $(c, \Phi)$.

\section{An extension of Thurston's Earthquake Theorem}

In this section we recall a recent result of [4] on constant curvature folations of MGHC AdS manifolds, and use it to prove Theorem 1.15.

\subsection{Constant curvature foliations in AdS geometry}

We recall here one of the main result of [4]. Let $N$ be a MGHC AdS 3-dimensional manifold, let $C(N)$ be its convex core.

Theorem 4.1 (Barbot, Béguin, Zeghib [4]) The complement of $C(N)$ in $N$ is foliated by surfaces of constant (Gauss) curvature. Moreover, for any $k \in(-\infty,-1)$, there exists a unique future-convex (resp. past-convex) surface of constant curvature $k$ in $N$, and it is a leaf of the foliation.

\subsection{Proof of Theorem $\mathbf{1 . 1 5}$}

We first translate Theorem 4.1 in terms of the landslide flow, using Lemma 1.9.

Corollary 4.2 Choose $\left(\rho_{l}, \rho_{r}\right) \in \mathcal{T}$ and $\alpha \in(0, \pi)$. There exists a unique element $\left(h, h^{\star}\right) \in \mathcal{T} \times \mathcal{T}$ such that

$$
L_{e^{i \alpha}}^{1}\left(h, h^{\star}\right)=\rho_{l}, \quad L_{e^{-i \alpha}}^{1}\left(h, h^{\star}\right)=\rho_{r} .
$$

Proof It is a direct consequence of Theorem 4.1. Given $\rho_{l}$ and $\rho_{r}$ there is a unique MGHC AdS manifold $N \cong S \times \mathbb{R}$ of which they are the left and right representations, respectively. By Theorem 4.1, $N$ contains a unique past-convex surface $F$ with constant curvature $-1 / \cos ^{2}(\alpha / 2)$, which comes with a diffeomorphism $\bar{\phi}: S \rightarrow F$ (well-defined up to isotopy). We call $I$ and $I I I$ the induced metric and third fundamental form on $S$, respectively. Then III has constant curvature $-1 / \sin ^{2}(\alpha / 2)$. We then set $h=\left(1 / \cos ^{2}(\alpha / 2)\right) I, h^{\star}=\left(1 / \sin ^{2}(\alpha / 2)\right) I I I$, so that $h$ and $h^{\star}$ are normalized 
hyperbolic metrics on $S$ (see [21]). Lemma 1.9 then shows that $\rho_{l}=L_{e^{i \alpha}}^{1}\left(h, h^{\star}\right)$, while $\rho_{r}=L_{e^{-i \alpha}}^{1}\left(h, h^{\star}\right)$.

Conversely, given $h, h^{\star} \in \mathcal{T}$ such that $\rho_{l}=L_{e^{i \alpha}}^{1}\left(h, h^{\star}\right)$, while $\rho_{r}=L_{e^{-i \alpha}}^{1}\left(h, h^{\star}\right)$, we can consider the unique equivariant embedding $\phi: \widetilde{S} \rightarrow \mathbb{A d S}^{3}$ onto a past-convex surface $\widetilde{F}$, with induced metric $\cos ^{2}(\alpha / 2) h$ and third fundamental form $\sin ^{2}(\alpha / 2) h^{\star}$. Then $\widetilde{F}$ is the lift to $\mathbb{A d S}^{3}$ of a past-convex surface $F$ in a MGHC AdS manifold $N$, and the left and right representations of $N$ are $\rho_{l}$ and $\rho_{r}$ by Lemma 1.9. This shows the uniqueness.

Proof of Theorem 1.15 Apply Corollary 4.2 with $\rho_{r}=h, \rho_{l}=h^{\prime}$, and with $\alpha=\theta / 2$. It shows there exists a unique $h_{0} \in \mathcal{T}$ and a unique $h_{0}^{\star} \in \mathcal{T}$ such that

$$
h=L_{e^{-i \alpha}}^{1}\left(h_{0}, h_{0}^{\star}\right), \quad h^{\prime}=L_{e^{i \alpha}}^{1}\left(h_{0}, h_{0}^{\star}\right) .
$$

As a consequence, putting $h^{\star}=L_{e^{-i \alpha}}^{2}\left(h_{0}, h_{0}^{\star}\right)$ we have $L_{e^{i \theta}}^{1}\left(h, h^{\star}\right)=h^{\prime}$.

Conversely given $h^{\star} \in \mathcal{T}$ such that $L_{e^{i \theta}}^{1}\left(h, h^{\star}\right)=h^{\prime}$, letting $\left(h_{0}, h_{0}^{\star}\right)=L_{e^{i \theta / 2}}\left(h, h^{\star}\right)$ we easily see that Equation (3) is verified. The uniqueness in Corollary 4.2 therefore implies the uniqueness here.

\section{The complex cyclic map}

This section describes a natural extension of the cyclic flow from a real to a complex parameter. This is analogous to the complex earthquake introduced by McMullen [28]. We will actually show in the next section that the "complex cyclic flow" introduced here limits, in a suitable sense, to the complex earthquake.

\subsection{Main statements}

Let $\mathcal{P}$ be the space of complex projective surface on $S$. The space $\mathcal{P}$ is naturally a complex manifold of real dimension $12 g-12$. Moreover the natural map $\mathcal{P} \rightarrow \mathcal{T}$ that associates to a complex projective surface the underlying complex structure is holomorphic. A projective structure is Fuchsian if its universal covering is projectively equivalent to a round disk in $\mathbb{C} \mathbb{P}^{1}$.

Let $\mathbb{H}$ be the upper half-plane in $\mathbb{C}$. We define a map

$$
P^{\prime}: \overline{\mathbb{H}} \times \mathcal{T} \times \mathcal{T} \rightarrow \mathcal{P}
$$

where

- for every fixed $h, h^{\star}$ the map $z \rightarrow P_{z}\left(h, h^{\star}\right)$ is holomorphic;

- for $t$ real, we have that $P_{t}^{\prime}\left(h, h^{\star}\right)$ is the Fuchsian projective surface corresponding to $L_{e^{-i t}}^{1}\left(h, h^{\star}\right)$. 
Notice that here $z$ lives in the upper half-plane, while, in the introduction, the flow usually depended on a complex parameter $\zeta$ in the unit disk. Both parameterizations are quite useful here. Taking $\zeta$ in the unit disk is natural when dealing with the landslide flow, while taking $z$ in the upper half-space is natural when thinking of complex earthquakes as a limit (since complex earthquakes are usually parameterized by the upper half-space). Until Section 5.4 we consider the parameterization by $z$ in the upper half-space, while in Section 5.5 we will make the connection to the parameterization by $\zeta$ in the unit disk. We use a prime to denote the various maps when $z$ is in the upper half-plane, this explains the notation $P^{\prime}$ above.

The construction of the map $P^{\prime}$ is the analog of the construction of the complex earthquake due to McMullen [28]. The first point is to define the analog of the grafting.

Given two normalized hyperbolic metrics $h$ and $h^{\star}$ on $\mathcal{T}$, let $b$ be the operator defined in Section 1.5. Given a positive number $s>0$, we consider the metric $I_{s}=\cosh ^{2}(s / 2) h$ and the operator $B_{s}=-\tanh (s / 2) b$. It is easy to see that $\left(I_{s}, B_{s}\right)$ satisfies the GaussCodazzi equation for immersed surfaces in $\mathbb{H}^{3}$, that is

$$
d^{\nabla} B_{s}=0, \quad K_{s}=-1+\operatorname{det} B_{s},
$$

where $\nabla$ is the Levi-Civita connection for $I_{S}$ (which is equal to the Levi-Civita connection for $h$ ) and $K_{S}$ is the curvature of $I_{S}$ (which is constant and equal to $\left.-1 / \cosh ^{2}(s / 2)\right)$.

As a consequence there exists a convex equivariant immersion

$$
\sigma_{s}: \widetilde{S} \rightarrow \mathbb{H}^{3}
$$

whose first fundamental form is the pullback $\tilde{I}_{S}$ of $I_{S}$ and whose shape operator is the pullback $\widetilde{B}_{S}$ of $B_{S}$. This map $\sigma_{s}$ is uniquely determined up to elements of $\operatorname{PSL}_{2}(\mathbb{C})$, once we state that the orientation on $\widetilde{S}$ at $\sigma_{s}(\tilde{p})$ coincides with the orientation induced by the normal vector $\widetilde{v}_{S}(\tilde{p})$ pointing towards the concave part (this is the reason why the sign of $B_{S}$ is negative).

Given $\tilde{p} \in \tilde{S}$, let $\operatorname{dev}_{S}(\widetilde{p}) \in S_{\infty}^{2}=\mathbb{C} \mathbb{P}^{1}$ be the endpoint of the geodesic ray starting from $\sigma_{s}(\tilde{p})$ with velocity $\widetilde{v}_{S}(\widetilde{p})$. The map

$$
\operatorname{dev}_{s}: \widetilde{S} \rightarrow \mathbb{C P}^{1}
$$

is a developing map for a complex projective structure $S G r_{S}^{\prime}\left(h, h^{\star}\right)$ on $S$. (The notation $S G r$ is used to keep in mind the analogy to the grafting map $G r$ ).

Notice that, if $h=h^{\star}$, then $b=E$ and $\sigma_{s}=d_{s} \circ \sigma_{0}$, where $d_{s}: \sigma_{0}(\tilde{S})=\mathbb{H}^{2} \rightarrow \mathbb{H}^{3}$ is the map associating to $\sigma_{s_{0}}(\tilde{p})$ the endpoint of the geodesic segment of length $s$ 
starting from $\sigma_{s_{0}}(\tilde{p})$ with velocity $\tilde{v}_{s_{0}}(\tilde{p})$. So, in this case, $S G r_{s}^{\prime}(h, h)$ is the Fuchsian projective structure associated to $h$.

Finally, given a complex number $z=t+i s$ with $s \geq 0$, we define

$$
P_{z}^{\prime}\left(h, h^{\star}\right)=S G r_{s}^{\prime}\left(L_{-t}^{\prime}\left(h, h^{\star}\right)\right),
$$

where $L_{-t}^{\prime}\left(h, h^{\star}\right):=L_{e^{-i t}}\left(h, h^{\star}\right)$.

Most of the remaining part of this section is devoted to proving the following theorem.

Theorem 5.1 The map

$$
z \mapsto P_{z}^{\prime}\left(h, h^{\star}\right) \in \mathcal{P}
$$

is holomorphic.

Composing $P^{\prime}$ with the forgetful map from $\mathcal{P}$ to $\mathcal{T}$, we obtain for each $z$ in the upper half-plane a map

$$
C_{z}^{\prime}: \mathcal{T} \times \mathcal{T} \rightarrow \mathcal{T}
$$

sending $\left(h, h^{\star}\right) \in \mathcal{T} \times \mathcal{T}$ to the complex structure underlying the complex projective structure $P_{z}^{\prime}\left(h, h^{\star}\right)$.

Corollary 5.2 The map $z \rightarrow C_{z}^{\prime}\left(h, h^{\star}\right)$ is holomorphic in the upper half-plane.

This clearly follows from Theorem 5.1 since the forgetful map from $\mathcal{P}$ to $\mathcal{T}$ is holomorphic.

To prove Theorem 5.1, we will show that the holonomy $\rho_{z}$ of $P_{z}^{\prime}$, holomorphically depends on $z$. In fact the derivatives

$$
\xi_{z}=\frac{\partial \rho_{z}}{\partial t}, \quad \eta_{z}=\frac{\partial \rho_{z}}{\partial s}
$$

are $\mathfrak{s l}_{2}(\mathbb{C})$-valued cocycles in $H^{1}\left(\pi_{1}(S), A d \circ \rho_{z}\right)$ and we will show that

$$
\eta_{z}=i \xi_{z}
$$

Let us remark that, since $P_{z+t}^{\prime}\left(h, h^{\star}\right)=P_{z}^{\prime}\left(L_{e^{-i t}}\left(h, h^{\star}\right)\right)$ for any $z$ in the upper plane and $t$ real, it is sufficient to verify Equation (5) at imaginary points $z_{0}=i s_{0}$.

To compute the cocycles we consider the family of convex immersions

$$
\sigma_{s}, \tau_{t}: \widetilde{S} \rightarrow \mathbb{H}^{3}
$$

such that $\sigma_{s}$ corresponds to $S G r_{s}^{\prime}\left(h, h^{\star}\right)$ and $\tau_{t}$ corresponds to $S G r_{s_{0}}^{\prime}\left(L_{e^{-i t}}\left(h, h^{\star}\right)\right)$. 
The first-order variations of $\sigma_{s}$ and $\tau_{t}$ are the fields

$$
X=\left.\frac{\partial \sigma_{s}}{\partial s}\right|_{s=s_{0}}, \quad Y=\left.\frac{\partial \tau_{t}}{\partial t}\right|_{t=0}
$$

regarded as sections of the fiber-bundle $\Theta=\sigma_{s_{0}}^{*}\left(T \mathbb{H}^{3}\right)$ on $\tilde{S}$. Imposing the equivariance of $\sigma_{s}$ under $\rho_{i s}$, and of $\tau_{t}$ under $\rho_{t+i s_{0}}$, we deduce that

$$
\begin{gathered}
X(\tilde{p})=(\rho(\gamma))_{*} X\left(\gamma^{-1} \tilde{p}\right)+\eta(\gamma)(\tilde{p}), \\
Y(\tilde{p})=(\rho(\gamma))_{*} Y\left(\gamma^{-1} \tilde{p}\right)+\xi(\gamma)(\tilde{p}),
\end{gathered}
$$

where we have put $\rho=\rho_{z_{0}}, \eta=\eta_{z_{0}}, \xi=\xi_{z_{0}}$ and we are identifying the elements of $\mathfrak{s l}_{2}(\mathbb{C})$ with the Killing vector fields on $\mathbb{H}^{3}$.

We will find some explicit relation between $X$ and $Y$ that, used in (7) and (8), will imply Equation (5).

Let us briefly explain the strategy of the computation. In Section 5.2 first we will decompose $D X$ into a self-adjoint part and a skew-symmetric. In particular the skewsymmetric part of $D X$ will be expressed in terms of cross-product with a new vector field $X^{\prime}$. Using this decomposition, we will point out some general equations relating the variation field $X$ and its derivatives to the variation of the first fundamental form and the shape operator. These equations are quite general and hold for any smooth family of immersions in $\mathbb{H}^{3}$. In particular those equations determine $X$ up to some global Killing vector fields.

In Section 5.3, comparing the equations satisfied by $X$ to the equations satisfied by $Y$, we will get that $Y=X^{\prime}$ up to a global Killing vector field.

In Section 5.4, we will achieve our goal by applying some classical construction to this relation.

\subsection{General formulas}

We consider any smooth family of immersions

$$
\sigma_{s}: \widetilde{S} \rightarrow \mathbb{H}^{3}
$$

and let $\widetilde{I}_{S}$ be the first fundamental form on $\widetilde{S}$ and $\widetilde{B}_{S}$ the shape operator associated with $\sigma_{s}$.

Fix $s_{0}>0$ : we study $\sigma_{s}$ around $s=s_{0}$. Let us denote by $\Theta$ the vector bundle $\sigma_{s_{0}}^{*}\left(T \mathbb{H}^{3}\right)$ and let $X=\left.(\partial \sigma / \partial s)\right|_{s=s_{0}}$, seen as a section of $\Theta$. In this section, we will express the derivatives of $\widetilde{I}_{s}$ and $\widetilde{B}_{S}$ at $s=s_{0}$ in terms of the field $X$, and we will show that these quantities determine $X$ up to global Killing vector fields. 
We notice that there is a natural inclusion of $T \widetilde{S}$ into $\Theta$ given by $d \sigma_{s_{0}}$. For the sake of simplicity, we will identify $T \widetilde{S}$ with its image in $\Theta$. Given a point $\widetilde{p} \in \tilde{S}$ and $s>0$ we denote by $\tilde{v}_{s}(\tilde{p})$ the unit vector at $\sigma_{s}(\tilde{p})$ orthogonal to $d \sigma_{s}(\tilde{p})\left(T_{\tilde{p}} \tilde{S}\right)$ such that, for every positive basis $\left\{e_{1}, e_{2}\right\}$ of $T_{\tilde{p}} \widetilde{S}$, the vectors $\widetilde{v}_{s}(\tilde{p}), d \sigma_{s}(\tilde{p})\left(e_{1}\right), d \sigma_{s}(\tilde{p})\left(e_{2}\right)$ form a positively oriented basis of $T_{\sigma_{s}}(\widetilde{p}) \mathbb{H}^{3}$. In this way, if $\widetilde{J}$ denotes the complex structure on $\widetilde{S}$ (and by abuse of notation on $\sigma_{S}(\tilde{S})$ ), we have

$$
\tilde{J}_{s} \tilde{v}=\tilde{v}_{s} \times \tilde{v}
$$

for all $\widetilde{v} \in d \sigma_{s}(T \widetilde{S})$.

A linear connection $D$ is defined on $\Theta$ by pulling back the Levi-Civita connection on $T \mathbb{H}^{3}$.

The covariant derivative of a section $Y$ of $\Theta$ is a linear operator

$$
D Y: T \widetilde{S} \rightarrow T \mathbb{H}^{3} .
$$

Such an operator can be decomposed as the sum of a self-adjoint operator of $T \widetilde{S}$ (identified to a subspace of $T \mathbb{H}^{3}$ ) and the restriction of a skew-symmetric operator of $T \mathbb{H}^{3}$.

Lemma 5.3 Given a section $V$ of $\Theta$, there exist

- a self-adjoint operator $A^{V}$ of $T \widetilde{S}$,

- a section $S^{V}$ of $\Theta$,

such that

$$
D_{\widetilde{v}} V=A^{V}(\widetilde{v})+S^{V} \times \widetilde{v}
$$

for every $\tilde{v} \in T \widetilde{S}$. Moreover both $A^{V}$ and $S^{V}$ are uniquely determined.

Proof At every point, $D V$ can be decomposed in a tangential part and normal part:

$$
D_{\widetilde{v}} V=\alpha(\widetilde{v})+\left\langle D_{\widetilde{v}} V, \widetilde{v}\right\rangle \widetilde{v} .
$$

Clearly $\alpha$ is an operator of $T \widetilde{S}$, so it can be decomposed into a self-adjoint part $A(\alpha)$ and a skew-symmetric part $S(\alpha)$. Notice that the skew-symmetric part is a multiple of $\widetilde{J}$, in particular there is $a \in \mathbb{R}$ such that $S(\alpha)(\widetilde{v})=a \widetilde{J}(\widetilde{v})=a \widetilde{v} \times \widetilde{v}$. On the other hand, there exists a tangent vector $\widetilde{w}$ such that

$$
\left\langle D_{\widetilde{v}} V, \widetilde{v}\right\rangle=\langle\widetilde{v}, \widetilde{w}\rangle
$$


for every $\widetilde{v} \in T \widetilde{S}$. In particular the normal part of $D_{\widetilde{v}} V$ can be regarded as the restriction on $T \widetilde{S}$ of the skew-symmetric operator

$$
\widetilde{v} \mapsto\langle\widetilde{v}, \widetilde{w}\rangle \widetilde{v}-\langle\widetilde{v}, \widetilde{v}\rangle \tilde{w}=(\widetilde{w} \times \widetilde{v}) \times \widetilde{v} .
$$

So if we put $A^{V}=A(\alpha)$ and $S^{V}=a \tilde{v}+(\widetilde{w} \times \widetilde{v})$, Equation (11) is verified.

We show now that this decomposition is unique. Suppose $A$ is a self-adjoint operator of $T \widetilde{S}$ and let $S$ be a vector tangent to $\mathbb{H}^{3}$ such that $A^{V}(\widetilde{v})+S^{V} \times \widetilde{v}=A(\widetilde{v})+S \times \widetilde{v}$ for all $\tilde{v} \in T \widetilde{S}$. Clearly $\left(A^{V}-A\right)(\widetilde{v})=\left(S-S^{V}\right) \times \widetilde{v}$. This implies that $S-S^{V}$ is a normal vector. Since $A-A^{V}$ is self-adjoint, it follows that $A^{V}-A=0$ and $S^{V}-S=0$.

An important property of the covariant derivative of $X$ is that $D_{\tilde{v}} X$ is the variation of the image of $\widetilde{v}$ in $\mathbb{H}^{3}$ along the family $\left(\sigma_{s}\right)$. More precisely the following statement holds. Here we denote by $D / d s$ the covariant derivative along $\left(\sigma_{s}\right)$ associated to $D$.

Lemma 5.4 Given a tangent vector $\widetilde{v} \in T_{\widetilde{p}} \widetilde{S}$, we consider the field $\widetilde{v}_{s}=d \sigma_{s}(\widetilde{v})$ along the curve $s \mapsto \sigma_{s}(\tilde{p})$. We have

$$
\left.\frac{D \widetilde{v}_{s}}{d s}\right|_{s=s_{0}}=D_{\widetilde{v}} X
$$

Proof Take a path $v:(-\delta, \delta) \rightarrow \widetilde{S}$ such that $v^{\prime}(0)=\widetilde{v}$ and consider the map $\tilde{x}(\varepsilon, s)=\sigma_{s}(v(\varepsilon))$. We have $\tilde{v}_{s}=\partial \tilde{x} / \partial \varepsilon(0, s)$ whereas $\partial \tilde{x} / \partial s\left(\varepsilon, s_{0}\right)=X(v(\varepsilon))$. A direct computation shows that

$$
\left.\frac{D}{d s} \widetilde{v}_{s}\right|_{s=s_{0}}=\frac{D}{d s} \frac{\partial \tilde{x}}{\partial \varepsilon}\left(0, s_{0}\right)=\frac{D}{d \varepsilon} \frac{\partial \tilde{x}}{\partial s}\left(0, s_{0}\right)=\frac{D}{d \varepsilon} X\left(0, s_{0}\right)=D_{\widetilde{v}} X .
$$

Now we apply the decomposition (11) to the field $X$, so we call $A^{X}$ the self-adjoint part of $D X$ and $X^{\prime}$ the field $S^{X}$. It turns out that the first order variation of $\widetilde{I}_{S}$ is determined by $A^{X}$. On the other hand, the field $X^{\prime}$ determines the variation of the normal field along the family of $\sigma_{s}$.

Lemma 5.5 Given $\tilde{u}, \widetilde{v} \in T_{\tilde{p}} \widetilde{S}$, we have

$$
\left.\frac{d}{d s} \tilde{I}_{s}(\widetilde{u}, \widetilde{v})\right|_{s=s_{0}}=2 \tilde{I}_{s}\left(A^{X}(\widetilde{u}), \widetilde{v}\right),\left.\quad \frac{D \widetilde{v}}{d s}\right|_{s=s_{0}}=X^{\prime} \times \widetilde{v} .
$$

Proof We have that

$$
\tilde{I}_{S}(\tilde{u}, \widetilde{v})=\left\langle d \sigma_{S}(\widetilde{u}), d \sigma_{s}(\widetilde{v})\right\rangle .
$$


Applying Lemma 5.4 we get

$$
\frac{d}{d s} \tilde{I}_{S}(\widetilde{u}, \widetilde{v})=\left\langle D_{\tilde{u}} X, \widetilde{v}\right\rangle+\left\langle\widetilde{u}, D_{\widetilde{v}} X\right\rangle .
$$

Since $D_{\tilde{u}} X=A^{X}(\tilde{u})+X^{\prime} \times \tilde{u}$, where $A^{X}$ is a self-adjoint operator of $T \widetilde{S}$, we get the first formula.

To prove the second formula, first we notice that since $\langle\widetilde{v}, \widetilde{v}\rangle=1$, then $\left.(D \widetilde{v} / d s)\right|_{s=s_{0}}$ is a tangent field. On the other hand, given a tangent vector $\widetilde{v}$ we have $\left\langle\widetilde{v}, d \sigma_{s}(\widetilde{v})\right\rangle=0$. Differentiating this identity we get

$$
\left\langle\frac{D \widetilde{v}}{d s}, \widetilde{v}\right\rangle=-\left\langle\widetilde{v}, D_{\widetilde{v}} X\right\rangle=\left\langle\widetilde{v}, X^{\prime} \times \widetilde{v}\right\rangle .
$$

Since $X^{\prime} \times \widetilde{v}$ is tangent, this proves that $D \widetilde{v} / d s=X^{\prime} \times \widetilde{v}$.

Using Equation (13) we get the first order variation of $\widetilde{B}_{S}$ at $s=s_{0}$. In the computation of such a variation, the covariant derivative of $X^{\prime}$ appears. It is useful to apply the decomposition (11) to $D X^{\prime}$. In particular, we put $X^{\prime \prime}=S^{X^{\prime}}$, so that

$$
D_{\widetilde{v}} X^{\prime}=A^{X^{\prime}}(\widetilde{v})+X^{\prime \prime} \times \widetilde{v} .
$$

Lemma 5.6 Given $\tilde{v} \in T_{\widetilde{p}} \widetilde{S}$, we have

$$
\left.\frac{d}{d s}\left(\widetilde{B}_{s}(\widetilde{v})\right)\right|_{s=s_{0}}=\widetilde{J} A^{X^{\prime}}(\widetilde{v})-\left\langle X+X^{\prime \prime}, \widetilde{v}\right\rangle \widetilde{v}-A^{X} \circ \widetilde{B}_{s_{0}}(\widetilde{v}) .
$$

Proof Differentiating with respect to $s$ the identity

$$
d \sigma_{s}\left(\widetilde{B}_{S}(\widetilde{v})\right)=-D_{\widetilde{v}} \tilde{v}
$$

and evaluating at $s=s_{0}$, we obtain

$$
D_{\widetilde{B}_{s_{0}}(\widetilde{v})} X+\dot{\tilde{B}}(\widetilde{v})=-\frac{D}{d s}\left(D_{\widetilde{v}} \widetilde{v}\right) .
$$

On the other hand, we have that

$$
\frac{D}{d s}\left(D_{\widetilde{v}} \widetilde{v}\right)=D_{\widetilde{v}}\left(\frac{D \widetilde{v}}{d s}\right)+\bar{R}(X, \widetilde{v}) \widetilde{v}
$$

where $\bar{R}$ is the Riemann tensor of $\mathbb{H}^{3}$.

By Equation (13) we have that

$$
D_{\widetilde{v}}\left(\frac{D \widetilde{v}}{d s}\right)=D_{\widetilde{v}}\left(X^{\prime} \times \widetilde{v}\right)=A^{X^{\prime}}(\widetilde{v}) \times \widetilde{v}+\left(X^{\prime \prime} \times \widetilde{v}\right) \times \widetilde{v}-X^{\prime} \times \widetilde{B}_{s_{0}}(\widetilde{v}) .
$$


On the other hand, since $\mathbb{H}^{3}$ has constant curvature -1, its Riemann tensor is simply given by

$$
\bar{R}(X, \widetilde{v}) \widetilde{v}=\langle X, \widetilde{v}\rangle \widetilde{v}-\langle\widetilde{v}, \widetilde{v}\rangle X=\langle X, \widetilde{v}\rangle \widetilde{v}
$$

Using (16), (17), and (18) in (15) we get $A^{X}\left(\widetilde{B}_{s_{0}}(\widetilde{v})\right)+X^{\prime} \times \widetilde{B}_{s_{0}}(\widetilde{v})+\dot{\widetilde{B}}(\widetilde{v})=-A^{X^{\prime}}(\widetilde{v}) \times \widetilde{v}-\left(X^{\prime \prime} \times \widetilde{v}\right) \times \widetilde{v}+X^{\prime} \times \widetilde{B}_{s_{0}}(\widetilde{v})-\langle X, \widetilde{v}\rangle \widetilde{v}$ Since $\widetilde{v} \times A^{X^{\prime}}(\widetilde{v})=\widetilde{J} A^{X^{\prime}}(\widetilde{v})$ and $\left(X^{\prime \prime} \times \widetilde{v}\right) \times \widetilde{v}=\left\langle X^{\prime \prime}, \widetilde{v}\right\rangle \widetilde{v}$, Equation (14) follows.

Finally we show that Equations (13) and (14) determine $X$ up to some global vector field. This is an easy consequence of the following lemma.

Lemma 5.7 Let $V$ be a section of $\Theta$ and let us put $V^{\prime}=S^{V}$ and $V^{\prime \prime}=S^{V^{\prime}}$. Suppose

$$
A^{V}=0, \quad \widetilde{J} A^{V^{\prime}}-\left\langle V^{\prime \prime}+V, \widetilde{v}\right\rangle E-A^{V} \circ \widetilde{B}_{s_{0}}=0 .
$$

Then $V$ is the restriction of a global Killing field of $\mathbb{H}^{3}$ on $\widetilde{S}$.

Proof Under the hypothesis of the lemma, neither the induced metric nor the shape operator of the surface vary under the first-order deformation defined by $V$. The conclusion therefore follows from the Fundamental Theorem of surface theory; see eg Spivak [40].

\subsection{The variation field of $S G r^{\prime}$}

In this section we apply formulas obtained in the previous subsection to the family of convex immersions $\sigma_{s}: \widetilde{S} \rightarrow \mathbb{H}^{3}$ defined in Equation (4).

Lemma 5.8 For $X=\left.(\partial \sigma / \partial s)\right|_{s=s_{0}}$, denote by $X^{\prime}$ the section $S^{X}$ and by $X^{\prime \prime}$ the section $S^{X^{\prime}}$. The following formulas hold:

$$
\begin{aligned}
2 A^{X} & =\tanh \left(s_{0} / 2\right) E ; \\
A^{X^{\prime}} & =[\tilde{J}, \tilde{b}] / 4 ; \\
\left\langle X+X^{\prime \prime}, \widetilde{v}\right\rangle & =\frac{\operatorname{tr}(\tilde{b})}{4} .
\end{aligned}
$$

Proof The embedding data corresponding to $\sigma_{s}$ are

$$
\tilde{I}_{s}=\cosh ^{2}(s / 2) \tilde{h}, \quad \widetilde{B}_{s}=-\tanh (s / 2) \tilde{b}
$$


so we easily get that

$$
\left.\frac{d}{d s} \widetilde{I}_{s}(\tilde{u}, \widetilde{v})\right|_{s=s_{0}}=\tanh \left(s_{0} / 2\right) \tilde{I}_{s_{0}}(\tilde{u}, \widetilde{v})
$$

Comparing this formula with Equation (13), we get that $2 A^{X}=\tanh \left(s_{0} / 2\right) E$.

On the other hand, applying Equation (14) we get

$$
-\frac{1}{2 \cosh ^{2}\left(s_{0} / 2\right)} \tilde{b}=\tilde{J} A^{X^{\prime}}-\left\langle X^{\prime \prime}+X, \tilde{v}\right\rangle E+\frac{\tanh ^{2}\left(s_{0} / 2\right)}{2} \tilde{b}
$$

which can be also written

$$
\tilde{b}=-2 \tilde{J} A^{X^{\prime}}+2\left\langle X^{\prime \prime}+X, \widetilde{v}\right\rangle E .
$$

Multiplying by $\tilde{J}$ we deduce that

$$
\widetilde{J} \tilde{b}=2 A^{X^{\prime}}+2\left\langle X^{\prime \prime}+X, \tilde{v}\right\rangle \tilde{J}
$$

Notice that this must coincide with the decomposition of $\widetilde{J} \tilde{b}$ in symmetric and skewsymmetric part. Since the adjoint of $\widetilde{J} \tilde{b}$ is $-\widetilde{b} \widetilde{J}$ it follows that

$$
\begin{gathered}
2 A^{X^{\prime}}=\frac{\tilde{J} \tilde{b}-\tilde{b} \widetilde{J}}{2}=\frac{[\tilde{J}, \tilde{b}]}{2}, \\
2\left\langle X^{\prime \prime}+X, \tilde{v}\right\rangle \widetilde{J}=\frac{\tilde{J} \tilde{b}+\tilde{b} \widetilde{J}}{2}=\frac{-\tilde{J} \tilde{b} \tilde{J}+\tilde{b}}{2} \tilde{J}=\frac{\tilde{b}^{-1}+\tilde{b}}{2} \widetilde{J}=\frac{\operatorname{tr}(\tilde{b})}{2} \tilde{J},
\end{gathered}
$$

where we have used that $\tilde{b}+\tilde{b}^{-1}=\operatorname{tr}(\tilde{b}) E$.

A consequence of Lemma 5.8 is that $X^{\prime \prime}+X$ can be explicitly computed.

Proposition 5.9 With the notation of Lemma 5.8 the following identity holds:

$$
X^{\prime \prime}=-X+\frac{\operatorname{tr}(\tilde{b})}{4} \tilde{v}
$$

Proof By Equation (21), it is sufficient to prove that

$$
\left\langle X^{\prime \prime}+X, \tilde{v}\right\rangle=0
$$

for every tangent vector $\tilde{v}$.

Let $\tilde{u}, \widetilde{v}$ be two tangent fields on $\widetilde{S}$. By using the identity

$$
D_{\tilde{u}} X=\frac{1}{2} \tanh \left(s_{0} / 2\right) \tilde{u}+X^{\prime} \times \tilde{u},
$$


an explicit computation shows that

$$
\bar{R}(\widetilde{u}, \widetilde{v}) X=D_{\widetilde{u}} D_{\widetilde{v}}(X)-D_{\widetilde{v}} D_{\widetilde{u}}(X)-D_{[\tilde{u}, \widetilde{v}]} X=D_{\widetilde{u}} X^{\prime} \times \widetilde{v}-D_{\widetilde{v}} X^{\prime} \times \tilde{u} .
$$

Moreover,

$$
D_{\tilde{u}} X^{\prime} \times \widetilde{v}-D_{\widetilde{v}} X^{\prime} \times \widetilde{u}=A^{X^{\prime}}(\widetilde{u}) \times \widetilde{v}-A^{X^{\prime}}(\widetilde{v}) \times \widetilde{u}+\left(X^{\prime \prime} \times \widetilde{u}\right) \times \widetilde{v}-\left(X^{\prime \prime} \times \widetilde{v}\right) \times \widetilde{u} .
$$

By Equation (20), $A^{X^{\prime}}$ is a self-adjoint traceless operator, and it follows that the sum of the first two terms of (22) vanishes. Eventually, we get

$$
\bar{R}(\widetilde{u}, \widetilde{v}) X=\left(X^{\prime \prime} \times \widetilde{u}\right) \times \widetilde{v}-\left(X^{\prime \prime} \times \widetilde{v}\right) \times \widetilde{u}=\left\langle X^{\prime \prime}, \widetilde{v}\right\rangle \tilde{u}-\left\langle X^{\prime \prime}, \tilde{u}\right\rangle \widetilde{v} .
$$

Since $\bar{R}(\widetilde{u}, \widetilde{v}) X=\langle X, \widetilde{u}\rangle \widetilde{v}-\langle X, \widetilde{v}\rangle \tilde{u}$, we easily deduce that $\left\langle X^{\prime \prime}+X, \widetilde{v}\right\rangle=0$ for all tangent vectors $\tilde{v}$.

Proposition 5.10 Let $\tau_{t}: \widetilde{S} \rightarrow \mathbb{H}^{3}$ be the family of convex immersions corresponding to the projective surface $S G r_{s_{0}}^{\prime} \circ L_{e^{-i t}}\left(h, h^{\star}\right)$ and denote by $Y$ its first order variation at $t=0$. Then $Y=-X^{\prime}$ up to adding a global Killing vector field, where $X^{\prime}$ is the vector field defined in Lemma 5.8.

Proof Let $\widetilde{I}_{t}$ be the first fundamental form corresponding to $\tau_{t}$ and let $\widetilde{B}_{t}$ be the corresponding shape operator. According to Lemmas 5.5, 5.6 and 5.7, it is sufficient to show that

$$
\begin{aligned}
\left.\frac{d}{d t} \widetilde{I}_{t}(\widetilde{u}, \widetilde{v})\right|_{t=0} & =-2 \widetilde{I}_{0}\left(A^{X^{\prime}}(\widetilde{u}), \widetilde{v}\right), \\
\left.\frac{d}{d t} \widetilde{B}_{t}(\widetilde{u})\right|_{t=0} & =-\left(\widetilde{J} A^{X^{\prime \prime}}(\widetilde{u})-\left\langle X^{\prime}+X^{\prime \prime \prime}, \widetilde{v}\right\rangle \tilde{u}-A^{X^{\prime}} \circ \widetilde{B}_{0}(\widetilde{u})\right),
\end{aligned}
$$

where $X^{\prime \prime \prime}=S^{X^{\prime \prime}}$ is the vector field corresponding to the skew-symmetric part of $D X^{\prime \prime}$. Call $\beta_{t}=\cos (t / 2) E-\sin (t / 2) J b$, so that we have

$$
\begin{aligned}
I_{t} & =\cosh ^{2}\left(s_{0} / 2\right) h\left(\beta_{t} \bullet, \beta_{t} \bullet\right), \\
B_{t} & =-\tanh \left(s_{0} / 2\right) \beta_{-t} b \beta_{t} .
\end{aligned}
$$

It follows that

$$
\begin{aligned}
\left.\frac{d}{d t} \tilde{I}_{t}(\tilde{u}, \widetilde{v})\right|_{t=0} & =\frac{1}{2}\left(-\tilde{I}_{0}(\tilde{J} \widetilde{b}(\widetilde{u}), \widetilde{v})-\tilde{I}_{0}(\tilde{u}, \tilde{J} \tilde{b}(\widetilde{v}))\right) \\
& =-\frac{1}{2} \widetilde{I}_{0}([\tilde{J}, \tilde{b}] \widetilde{u}, \widetilde{v})=-2 \widetilde{I}_{0}\left(A^{X^{\prime}}(\widetilde{u}),(\widetilde{v})\right)
\end{aligned}
$$


To prove Equation (24), we first compute $D X^{\prime \prime}$. By Proposition 5.9 we have

$$
\begin{aligned}
D_{u} X^{\prime \prime} & =-A^{X} u-X^{\prime} \times u-\frac{\operatorname{tr}(\widetilde{b})}{4} \widetilde{B}_{0} u+\left\langle\operatorname{grad}\left(\frac{\operatorname{tr}(\tilde{b})}{4}\right), u\right) \tilde{v} \\
& =-\left(A^{X}+\frac{\operatorname{tr}(\tilde{b})}{4} \widetilde{B}_{0}\right) u-\left(X^{\prime}+\widetilde{J} \operatorname{grad}(\operatorname{tr}(\widetilde{b}) / 4)\right) \times u .
\end{aligned}
$$

where grad is the gradient on $\widetilde{S}$ with respect to $\widetilde{I}_{0}$. In particular,

$$
X^{\prime \prime \prime}=-X^{\prime}-\tilde{J} \operatorname{grad}(\operatorname{tr}(\tilde{b}) / 4), \quad 2 A^{X^{\prime \prime}}=-\tanh \left(s_{0} / 2\right)\left(E-\frac{\operatorname{tr}(\tilde{b})}{2} \tilde{b}\right) .
$$

Replacing these identities in the right hand side of (24), we deduce

$$
\begin{aligned}
& 2\left(\widetilde{J} A^{X^{\prime \prime}}(\widetilde{u})-\left\langle X^{\prime}+X^{\prime \prime \prime}, \widetilde{v}\right\rangle \tilde{u}-A^{X^{\prime}} \circ \widetilde{B}_{0}(\widetilde{u})\right) \\
& =-\tanh \left(s_{0} / 2\right)\left(\tilde{J}-\frac{\operatorname{tr}(\tilde{b})}{2} \tilde{J} \widetilde{b}\right)(\widetilde{u})+\tanh \left(s_{0} / 2\right) \frac{(\tilde{J} \tilde{b}-\tilde{b} \widetilde{J})}{2} \tilde{b}(\tilde{u}) \\
& =-\tanh \left(s_{0} / 2\right)\left(\widetilde{J}-\frac{\operatorname{tr} \tilde{b}}{2} \widetilde{J} \widetilde{b}-\frac{1}{2} \widetilde{J} \widetilde{b}^{2}+\frac{\widetilde{J}}{2}\right)(\widetilde{u}) .
\end{aligned}
$$

Using the identity $\widetilde{b}^{2}=\operatorname{tr}(\tilde{b}) \tilde{b}-E$, we have that the right hand side in (24) is equal to

$$
\tanh \left(s_{0} / 2\right)(\tilde{J}-(\operatorname{tr}(\tilde{b}) / 2) \tilde{J} \tilde{b}) .
$$

On the other hand, Equation (26) shows that the left hand side of (24) is equal to

$$
\begin{aligned}
\frac{\tanh \left(s_{0} / 2\right)}{2}\left(\tilde{b} \tilde{J} \tilde{b}-\tilde{J} \widetilde{b}^{2}\right) & =\frac{\tanh \left(s_{0} / 2\right)}{2}(\tilde{J}-\tilde{J}(\operatorname{tr}(\tilde{b}) \tilde{b}-E)) \\
& =\frac{\tanh \left(s_{0} / 2\right)}{2}(2 \tilde{J}-\operatorname{tr}(\tilde{b}) \tilde{J} \tilde{b}) .
\end{aligned}
$$

Equation (24) follows by comparing (27) with (28).

\subsection{The comparison of the cocycles}

Any element of $K \in \mathfrak{s l}_{2}(\mathbb{C})$ can be regarded as a Killing vector field on $\mathbb{H}^{3}$. Notice that by definition of Killing vector field, there is another field $K^{\prime}$ associated to $K$ so

$$
D_{\tilde{v}} K=K^{\prime} \times \widetilde{v} .
$$

The field $K^{\prime}$ is a Killing vector field and in fact $K^{\prime \prime}=-K$; see Hodgson and Kerckhoff [16]. More precisely the following lemma holds.

Lemma 5.11 [16] As elements of $\mathfrak{s l}_{2}(\mathbb{C})$ we have $K^{\prime}=i K$. 
Given a point $\tilde{x} \in \mathbb{H}^{3}$ we have a natural map

$$
\operatorname{ev}_{\tilde{x}}: \mathfrak{s l}_{2}(\mathbb{C}) \ni K \mapsto\left(K(\tilde{x}), K^{\prime}(\tilde{x})\right) \in T_{\widetilde{x}} \mathbb{H}^{3} \oplus T_{\widetilde{x}} \mathbb{H}^{3} .
$$

It is a well-known fact that such a map is an isomorphism for every $\tilde{x} \in \mathbb{H}^{3}$.

Because of Lemma 5.11, if $\operatorname{ev}_{\tilde{x}}(K)=\left(\widetilde{w}_{1}, \widetilde{w}_{2}\right)$, then $\operatorname{ev}_{\tilde{x}}(i K)=\left(\widetilde{w}_{2},-\widetilde{w}_{1}\right)$.

Given any section $V$ on $\Theta$, we define

$$
K^{V}: \widetilde{S} \rightarrow \mathfrak{s l}_{2}(\mathbb{C})
$$

such that $\operatorname{ev}_{\tilde{x}}\left(K^{V}(\tilde{p})\right)=V(\tilde{p})$ and $\operatorname{ev}_{\tilde{x}}\left(\left(K^{V}\right)^{\prime}(\tilde{p})\right)=V^{\prime}(\tilde{p})$ for every $\tilde{x}=\sigma_{s_{0}}(\tilde{p})$.

In particular, we have maps $K^{X}$ and $K^{Y}$ associated to the fields $X, Y$ defined in Equation (6), so that $\operatorname{ev}_{\tilde{x}}\left(K^{X}\right)=\left(X, X^{\prime}\right)$ and $\operatorname{ev}_{\tilde{x}}\left(K^{Y}\right)=\left(-X^{\prime},-X^{\prime \prime}\right)$ by Proposition 5.10. We conclude by Proposition 5.9 that

$$
K^{Y}=-i K^{X}-K_{0},
$$

where $K_{0}(\tilde{p})=\operatorname{ev}_{\tilde{x}}^{-1}\left(0, X^{\prime \prime}+X\right)=\operatorname{ev}_{\tilde{x}}^{-1}(0, \operatorname{tr}(\tilde{b}) / 4 \widetilde{v})$ for all $\tilde{x}=\sigma_{s_{0}}(\tilde{p})$. Since the field $\operatorname{tr}(\widetilde{b}) / 4 \widetilde{v}$ is invariant under the action of $\pi_{1}(S)$, we find that $K_{0}$ is equivariant, that is

$$
K_{0}(\gamma \tilde{p})=A d(\rho(\gamma)) K_{0}(\tilde{p}) .
$$

However, it follows from Equation (7) that

$$
\begin{gathered}
K^{X}(\gamma \tilde{p})-A d(\rho(\gamma)) K^{X}(\tilde{p})=\eta(\gamma), \\
K^{Y}(\gamma \tilde{p})-A d(\rho(\gamma)) K^{Y}(\tilde{p})=\xi(\gamma),
\end{gathered}
$$

and by these equations and identities (7) and (8) one deduces that

$$
\xi(\gamma)=-i \eta(\gamma)-(A d(\rho(\gamma))-1) K_{0}(\tilde{p})=-i \eta(\gamma) .
$$

Thus Equation (5) is proved.

\subsection{Parameterization by the disk}

The parameterization of the complex landslide used above is well-suited for a comparison with the complex earthquake. However, another parameterization-already used in the introduction-is perhaps more convenient when considering the holomorphic disks in Teichmüller space obtained as the image of the complex flow. This new parameter $\zeta$ takes values in the unit disk. We develop here the relationship between these two parameterizations and we investigate the regularity at $\zeta=0$. 
Consider $z=t+i s$ in the upper half-plane, so that $t \in \mathbb{R}$ and $s \geq 0$, and we set $\zeta=\exp (i z)=\exp (-s+i t)$, which belongs to the punctured closed unit disk $\dot{\bar{\Delta}}=\{\zeta \in \mathbb{C}|0<| \zeta \mid \leq 1\}$. For $h, h^{\star} \in \mathcal{T}$ we then define

$$
P_{\zeta}\left(h, h^{\star}\right):=P_{t+i s}^{\prime}\left(h, h^{\star}\right), \quad C_{\zeta}\left(h, h^{\star}\right):=C_{t+i s}^{\prime}\left(h, h^{\star}\right),
$$

where $P_{t+i s}^{\prime}\left(h, h^{\star}\right)$ is the projective structure $S G r_{s}^{\prime}\left(L_{-t}^{\prime}\left(h, h^{\star}\right)\right)$ and $C_{t+i s}^{\prime}\left(h, h^{\star}\right)$ is the underlying conformal structure. These maps are well-defined since $P^{\prime}$ and $C^{\prime}$ are invariant under $t \mapsto t+2 \pi$. Clearly, the maps $\zeta \mapsto P_{\zeta}\left(h, h^{\star}\right)$ and $\zeta \mapsto C_{\zeta}\left(h, h^{\star}\right)$ are holomorphic in the unit disk minus its center, for any fixed $h$ and $h^{\star}$.

We first give an explicit formula for $C_{i s}^{\prime}\left(h, h^{\star}\right)$.

Lemma 5.12 Let $h, h^{\star} \in \mathcal{T}$ be two hyperbolic metrics on $S$, and let $b$ be the bundle morphism appearing in Corollary 1.5. For every $s \in \mathbb{R}_{\geq 0}$,

$$
C_{i s}^{\prime}\left(h, h^{\star}\right)=h\left(\gamma_{s} \bullet, \gamma_{s} \bullet\right)
$$

as conformal structures, where

$$
\gamma_{s}=\cosh (s / 2) E+\sinh (s / 2) b .
$$

Proof By definition, $C_{i s}^{\prime}\left(h, h^{\star}\right)$ is the conformal structure at infinity of the (unique) hyperbolic end containing a convex surface with induced metric $I=\cosh ^{2}(s / 2) h$ and third fundamental form $I I I=\sinh ^{2}(s / 2) h^{\star}$. Its shape operator is then $B=\tanh (s / 2) b$, and conformal structure at infinity of the end is given (see eg the third author [37]) by

$$
C_{i s}^{\prime}\left(h, h^{\star}\right)=I((E+B) \bullet,(E+B) \bullet)=h\left(\gamma_{s} \bullet, \gamma_{s} \bullet\right) .
$$

We can now give a general formula for $C_{t+i s}^{\prime}\left(h, h^{\star}\right)$ for $t+i s \in \overline{\mathbb{H}}$.

Lemma 5.13 Let $s, t \in \mathbb{R}$ with $s \geq 0$, and let $\zeta=\exp (-s+i t)$. Then, for all $h, h^{\star} \in \mathcal{T}$,

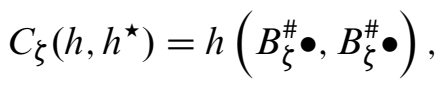

where

$$
B_{\zeta}^{\#}=\frac{\zeta+1}{2 \sqrt{\zeta}} E-\frac{\zeta-1}{2 \sqrt{\zeta}} b
$$

and $\sqrt{\zeta}$ is a notation for $\exp ((-s+i t) / 2)$.

Here we use the convention that the complex number $i$ acts as the complex structure $J$ on tangent vectors. 
Proof It follows from the definition of $C^{\prime}$ and from the previous lemma that

$$
C_{t+i s}^{\prime}\left(h, h^{\star}\right)=h\left(\beta_{-t} \circ \bar{\gamma}_{s} \bullet, \beta_{-t} \circ \bar{\gamma}_{s} \bullet\right),
$$

where (as in Section 3)

$\beta_{t}=\cos (t / 2) E+\sin (t / 2) J b, \bar{\gamma}_{s}=\cosh (s / 2) E+\sinh (s / 2) b_{-t}, b_{-t}=\beta_{t} \circ b \circ \beta_{-t}$.

It is then clear that

$$
\beta_{-t} \circ \bar{\gamma}_{s}=\gamma_{s} \circ \beta_{-t},
$$

so that

$$
C_{t+i s}^{\prime}\left(h, h^{\star}\right)=h\left(B_{\zeta}^{\#} \bullet, B_{\zeta}^{\#} \bullet\right)
$$

where $B_{\zeta}^{\#}=\gamma_{s} \circ \beta_{-t}$.

Using the fact that $b J b J=-E$,

$$
\begin{aligned}
& B_{\zeta}^{\#}=(\cosh (s / 2) E+\sinh (s / 2) b) \circ(\cos (t / 2) E-\sin (t / 2) J b) \\
&=(\cosh (s / 2) \cos (t / 2) E-\sinh (s / 2) \sin (t / 2) b J b) \quad+(\cos (t / 2) \sinh (s / 2) E-\cosh (s / 2) \sin (t / 2) J) b \\
&=(\cosh (s / 2) \cos (t / 2) E-\sinh (s / 2) \sin (t / 2) J) \\
& \quad+(\cos (t / 2) \sinh (s / 2) E-\cosh (s / 2) \sin (t / 2) J) b \\
&=\cosh ((-s+i t) / 2) E-\sinh ((-s+i t) / 2) b .
\end{aligned}
$$

Setting $\sqrt{\zeta}=\exp ((-s+i t) / 2)$, we can write this relation as

$$
B_{\zeta}^{\#}=\frac{\sqrt{\zeta}+1 / \sqrt{\zeta}}{2} E-\frac{\sqrt{\zeta}-1 / \sqrt{\zeta}}{2} b
$$

It follows from the definitions that $C$ is essentially the same as $C^{\prime}$ with a different parameterization. The main properties of this map are as follows.

Proposition 5.14 Let $h, h^{\star} \in \mathcal{T}$ and let $c$ be the "center" of $\left(h, h^{\star}\right)$ as defined in Section 1.8. Then

(1) $C_{\zeta}\left(h, h^{\star}\right)$ is defined for all $\zeta \in \dot{\bar{\Delta}}$,

(2) it is holomorphic in $\zeta$,

(3) it extends continuously, and thus holomorphically, at $\zeta=0$, with $C_{0}\left(h, h^{\star}\right)=c$,

(4) it extends holomorphically to the open disk of center 0 and radius $\left(\kappa_{0}+1\right) /\left(\kappa_{0}-1\right)$, where $\kappa_{0}=\max _{x \in S} \kappa(x)$ and $\kappa: S \rightarrow[1, \infty)$ is the bigger eigenvalue of the operator $b$ associated to the minimal Lagrangian map isotopic to the identity between $(S, h)$ and $\left(S, h^{\star}\right)$ (see Section 1.5). 
In particular, $c$ appears as a smooth point of the holomorphic disk defined by $C$, while it was obtained only in the limit $s \rightarrow \infty$ in the parameterization used by $C^{\prime}$.

Remark 5.15 Unlike the map $C_{\bullet}\left(h, h^{\star}\right)$, the map $P_{\bullet}\left(h, h^{\star}\right)$ does not extend at $\zeta=0$. Indeed, take any sequence of positive real numbers $\zeta_{n} \rightarrow 0$. By definition of the map $P$, there is an embedding of $S$ into the hyperbolic end $M_{n}$, with first fundamental form equal to $I_{n}=\cosh ^{2}\left(-\frac{1}{2} \log \zeta_{n}\right) h$ and shape operator $B_{n}=-\tanh \left(-\frac{1}{2} \log \zeta_{n}\right) b$, which corresponds to the projective structure $P_{n}$. In particular, $B_{n}$ converges to $-b$. On the other hand, by the proof by Labourie of Proposition 4.2 of [24], if $P_{n}$ converges to a projective surface, $B_{n}$ should converge to the identity.

Proof of Proposition 5.14 The first two points are direct consequences of the definition of $C$ from $C^{\prime}$, and of Theorem 5.1. The third point follows from the expression of $B_{\zeta}^{\#}$ in Lemma 5.13, because $C_{\zeta}\left(h, h^{\star}\right)$ is really considered as a conformal structure, so it is not changed if we multiply $B_{\zeta}^{\#}$ by a complex-valued function defined on $S$. In particular, we can multiply $B_{\zeta}^{\#}$ by $2 \sqrt{\zeta}$, obtaining

$$
2 \sqrt{\zeta} B_{\zeta}^{\#}=(1+\zeta) E+(1-\zeta) b,
$$

which is clearly continuous at $u=0$.

For the last point note that the expression defining $C_{\zeta}\left(h, h^{\star}\right)$ in Lemma 5.13 can be analytically continued if $B_{\zeta}^{\#}$ is nonsingular at all points of $S$. This happens if

$$
\frac{\zeta+1}{2 \sqrt{\zeta}}+\frac{1-\zeta}{2 \sqrt{\zeta}} \kappa \neq 0
$$

everywhere on $S$, which is certainly satisfied if

$$
|\zeta|<\frac{\kappa_{0}+1}{\kappa_{0}-1}
$$

\section{The earthquake flow as a limit}

The main goal of this section is to prove Theorems 1.12 and 1.14. The arguments are based on comparing surfaces in $\mathbb{A d S}^{3}$ with constant Gauss curvature close to -1 to pleated surfaces. The key step in the proof of Theorem 1.12 will be Theorem 1.14.

We fix a hyperbolic metric $h$ on $S$ and a divergent sequence of metrics $h_{n}^{\star} \in \mathcal{T}$. We will study the asymptotic behavior of the holomorphic map $z \mapsto P_{z}\left(h, h_{n}^{\star}\right)$ assuming that $\left(h_{n}^{\star}\right)_{n \in \mathbb{N}}$ converges to a point in the Thurston boundary of $\mathcal{T}(S)$ which is the projective class of some measured geodesic lamination $\lambda$ on $S$. 
Take any sequence $\theta_{n}>0$ with $\lim _{n \rightarrow \infty} \theta_{n}=0$ such that $\theta_{n} \ell_{h_{n}^{\star}} \rightarrow \iota(\lambda, \bullet)$, ie for every free homotopy class $\gamma$ of closed curves of $S$, the $h_{n}^{\star}$-length of the $h_{n}^{\star}$-geodesic representative of $\gamma$ rescaled by the factor $\theta_{n}$ converges to the intersection between $\gamma$ and $\lambda$. Define $P_{n}^{\prime}: \overline{\mathbb{H}} \rightarrow \mathcal{P}(S)$ as $P_{n}^{\prime}(z)=P_{\theta_{n} z}^{\prime}\left(h, h_{n}^{\star}\right)$, which are holomorphic, and let $P_{\infty}^{\prime}: \overline{\mathbb{H}} \rightarrow \mathcal{P}(S)$ be $P_{\infty}^{\prime}(t+i s)=G r_{s \lambda / 2}\left(E_{-t \lambda / 2}(h)\right)$.

Theorem 6.1 For every $z \in \overline{\mathbb{H}}$, we have that $P_{n}^{\prime}(z) \rightarrow P_{\infty}^{\prime}(z)$.

Notice that since the $P_{n}^{\prime}$ are holomorphic, the convergence $P_{n}^{\prime} \rightarrow P_{\infty}^{\prime}$ is, in fact, in $C^{\infty}$. Note also that in this section we use the parameterization by the upper halfplane-which is more practical when considering the limit to complex earthquakes-so that we use the notation with primes for $L, P, S G r$, etc.

Outline of the section Since the arguments of this section are quite long, technical and sometimes involved, we include an outline in order to help the reader.

In Section 6.1 we consider first the convergence issue in Theorem 6.1 for $z$ imaginary. This means that we prove the convergence of the smooth grafting map $S G r$ to the "usual" grafting map $\mathrm{Gr}$.

This convergence issue can be stated in terms of hyperbolic 3-dimensional geometry: if a sequence of hyperbolic ends $\left(M_{n}\right)_{n \in \mathbb{N}}$ contains a sequence of $K$-surfaces with $K \rightarrow-1$, with induced metrics proportional to a fixed hyperbolic metric $h$, and with third fundamental forms converging projectively to a measured lamination $\lambda$, then $\left(M_{n}\right)_{n \in \mathbb{N}}$ converges to a hyperbolic end for which the pleated surface boundary has induced metric $h$ and measured bending lamination $\lambda$.

We then use the duality between $\mathbb{H}^{3}$ and the de Sitter space $\mathrm{dS}^{3}$ to turn this hyperbolic convergence problem into a convergence question for $K$-surfaces close to the initial singularity in MGHC de Sitter spaces. There we can use a convergence result obtained by M. Belraouti [5] first in the flat case, and recently extended [6] to the de Sitter case, and conclude to the convergence of Theorem 6.1 for imaginary $z$.

In Section 6.2 we turn to the convergence in Theorem 1.12, but now on the real axis. This section only considers the convergence of the first factor in the landslide map $L_{\theta_{n}}^{\prime}$ to a limit hyperbolic metric, while the convergence of the second factor (suitably normalized) to a measured lamination is dealt with in Sections 6.3 and 6.4.

This convergence can again be stated in terms of 3-dimensional geometry, but now for AdS manifolds rather than hyperbolic ends; see Proposition 6.9. We consider a sequence of AdS MGHC manifolds $N_{n}$ containing a future-convex surface $F_{n}$ with induced metric proportional to $h$ and third fundamental form proportional to $h_{n}^{\star}$, as 
the curvature tends to -1 , and need to prove that the $N_{n}$ converge to a limit MGHC manifold $N_{\infty}$ and that the $F_{n}$ converge to the past boundary of the convex core of $N_{\infty}$. To prove this we consider the universal covers $\widetilde{F}_{n}$ of the $F_{n}$ as space-like surfaces in $\mathbb{A d S}^{3}$, invariant under surface group actions, and need to prove that they converge to a space-like, pleated surface.

The difficulty here is to prove that the limit surface is space-like rather than lightlike. The proof uses an interplay between the AdS and the hyperbolic 3-dimensional representations, in particular it is based on Lemma 6.11, which has a hyperbolic character. This lemma provides an upper bound on the principal curvatures of the surfaces $\widetilde{F}_{n}$, from which it is possible to prove that the surfaces remain "uniformly space-like". The convergence of the representations also follows.

In Section 6.3 we turn to the convergence of the second factor in Theorem 1.12. This section contains the proof of a statement which is perhaps of independent interest. Suppose again that $h$ is fixed and that $\theta_{n} \ell_{h_{n}^{\star}}$ converges to $\iota(\lambda, \bullet)$ on closed curves, and let $b_{n}$ be the operator expressing $h_{n}^{\star}$ in terms of $h$ (as in Corollary 1.5). Then we prove that the sequence of measures $\operatorname{tr}\left(b_{n}\right) \omega_{h_{n}}$ converges to the uniform measure on the geodesic lamination realizing $\lambda$ in $(S, h)$ (see Proposition 6.16). The proof takes place in 3-dimensional hyperbolic ends again, and is based on a more precise understanding of the convergence of a sequence of $K$-surfaces to a pleated surface as $K$ tends to -1 .

A direct consequence (Corollary 6.19) is that $\left(\theta_{n} \ell_{h_{n}^{\star}}(\gamma)\right)_{n \in \mathbb{N}}$ is bounded for every $\gamma \in \pi_{1}(S)$ if and only if $\theta_{n} \int_{S} \operatorname{tr}\left(b_{n}\right) \omega_{h_{n}}$ is bounded. This is used in Section 6.4 to prove the convergence of the second factor in Theorem 1.12, for $z$ on the real axis, and to conclude the proof of this theorem.

Finally, Section 6.5 is devoted to the proof of Theorem 1.14. The proof again takes place in 3-dimensional hyperbolic ends, or actually-this is possible thanks to a scaling trick-in quasi-Fuchsian manifolds. We consider again a sequence of $K$-surfaces with induced metrics proportional to a fixed hyperbolic metric $h$, and third fundamental forms proportional to a sequence of hyperbolic metrics $h_{n}$ limiting to a measured lamination, when the curvatures go to -1 . At this point we will have already proved that those $K$-surfaces then converge to a pleated surface in a quasi-Fuchsian manifold, and Theorem 1.14 can be translated as saying that the third fundamental forms of those surfaces, suitably normalized, converge in a strong sense to the intersection with the measured bending lamination of the limit bent surface.

The key technical point is now to get a good control of the convergence of the support planes of the $K$-surfaces to the support planes of the pleated surface (see in particular the proof of Lemma 6.21). The proof of those convergence properties again use the 
duality between the hyperbolic and the de Sitter space. The proof then proceeds by comparison between the length of a curve $\alpha$ on the dual of the pleated surfacecorresponding to the intersection of $\alpha$ with the pleating lamination-and the de Sitter distance between the points in de Sitter dual to the support planes at the endpoints of $\alpha$, using in particular geometric estimates in the de Sitter space.

\subsection{Convergence on the imaginary axis}

In this subsection we prove that if $\theta_{n} \ell_{h_{n}^{\star}} \rightarrow \iota(\lambda, \bullet)$, then

$$
P_{i \theta_{n}}^{\prime}\left(h, h_{n}^{\star}\right) \rightarrow G r_{\lambda / 2}(h) \text {. }
$$

In fact, in order to prove Theorem 6.1, we will need to prove that the convergence is uniform with respect to $h$.

Proposition 6.2 Let $\left(h_{n}\right)_{n \in \mathbb{N}}$ be a sequence of hyperbolic metrics converging to a hyperbolic metric $h$ on $S$, and let $\left(h_{n}^{\star}\right)_{n \in \mathbb{N}}$ be a sequence of hyperbolic metrics converging projectively to $[\lambda]$ in the Thurston boundary of $\mathcal{T}$. If $\theta_{n}$ is a sequence of positive numbers such that $\theta_{n} \ell_{h_{n}^{\star}} \rightarrow \iota(\lambda, \bullet)$, then $S G r_{\theta_{n}}^{\prime}\left(h_{n}, h_{n}^{\star}\right)$ converges to $G r_{\lambda / 2}(h)$.

Notice that (31) corresponds to the particular case of Proposition 6.2 in which $h_{n}$ is constant.

By definition, $S G r_{\theta_{n}}^{\prime}\left(h_{n}, h_{n}^{\star}\right)=P_{i \theta_{n}}^{\prime}\left(h_{n}, h_{n}^{\star}\right)$ is the projective structure on $S$ determined by prescribing that the associated hyperbolic end $\left(M_{n}, g_{M_{n}}\right)$ contains a constant curvature surface $S_{n}$, parametrized by $\bar{\sigma}_{n}: S \rightarrow S_{n} \subset M_{n}$, with first fundamental form $I_{S_{n}}=\cosh ^{2}\left(\theta_{n} / 2\right) h_{n}$ and third fundamental form $I I I_{S_{n}}=\sinh ^{2}\left(\theta_{n} / 2\right) h_{n}^{\star}$.

In general, given an embedding $S \rightarrow \Sigma \subset X$ of $S$ inside a (hyperbolic, de Sitter or anti-de Sitter) 3-manifold, we will denote by the same symbol the first fundamental form $I_{\Sigma}$ (resp. the third fundamental form $I I I_{\Sigma}$ ) and its pullback on $S$.

Let $\partial M_{n}$ be the hyperbolic boundary of $M_{n}$, that carries a hyperbolic induced metric $g_{\partial M_{n}}$ and is locally bent along a measured geodesic lamination $\lambda_{n}$. By definition, $P_{i \theta_{n}}^{\prime}\left(h_{n}, h_{n}^{\star}\right)=G r_{\lambda_{n}}\left(g_{\partial M_{n}}\right)$. So, in order to prove Proposition 6.2, it is sufficient to check that $\left(S, g_{\partial M_{n}}\right)$ converges to $(S, h)$, and $\lambda_{n}$ converges to $\lambda / 2$ in $\mathcal{M L}(S)$.

Lemma 6.3 The hyperbolic metrics $g_{\partial M_{n}}$ are contained in a compact subset of $\mathcal{T}$.

Proof The closest point projection $r_{n}: M_{n} \rightarrow \partial M_{n}$ is 1-Lipschitz. In particular, we have a $1-$ Lipschitz map $\left.r_{n}\right|_{S_{n}}: S_{n} \rightarrow \partial M_{n}$. This implies that the marked length spectrum of $\partial M_{n}$ is bounded from above by the marked length spectrum of $S_{n}$, that in turn is locally uniformly bounded. 
Lemma 6.4 The bending laminations $\lambda_{n}$ are contained in a compact subset of $\mathcal{M L}$.

In order to prove Lemma 6.4, we will consider the de Sitter spacetime $M_{n}^{\star}$ dual to $M_{n}$ : it is the set of complete geodesic planes contained in $M_{n}$. The de Sitter structure is induced by the natural map $\operatorname{dev}^{\star}: \widetilde{M}_{n}^{\star} \rightarrow \mathrm{dS}^{3}$, where the model of de Sitter geometry is the set of geodesic planes of $\mathbb{H}^{3}$ (see Scannell [34] and Benedetti and the first author [7]).

Scannell [34] showed that $M_{n}^{\star}$ is a MGHC spacetime diffeomorphic to $S \times \mathbb{R}$. Following [7], $\widetilde{M}_{n}^{\star}$ has a natural boundary corresponding to the set of support planes of $\partial \widetilde{M}_{n}$. This boundary is called the initial singularity: the de Sitter metric extends to the boundary and makes it an achronal (but not spacelike) surface. So $\partial \widetilde{M}_{n}^{\star}$ carries a pseudometric $d_{0}$ induced by $\widetilde{M}_{n}^{\star}$. By [7] and Benedetti and Guadagnini [8] it turns out that the action of $\pi_{1}(S)$ on $\widetilde{M}_{n}^{\star}$ extends to the boundary (even if the action on the boundary is neither proper nor free) and the marked length spectrum of this action coincides with the intersection with the bending lamination:

$$
\iota\left(\lambda_{n}, \gamma\right)=\inf \left\{d_{0}\left(x^{\star}, \gamma x^{\star}\right) \mid x^{\star} \in \partial \widetilde{M}_{n}^{\star}\right\} .
$$

Let $S_{n}^{\star}$ be the surface in $M_{n}^{\star}$ dual to $S_{n}$, corresponding to the set of support planes of $S_{n}$ (that by the convexity of $S_{n}$ are complete planes in $M_{n}$ ). There is a natural map $S_{n} \rightarrow S_{n}^{\star}$ sending $x$ to the dual of the plane tangent to $S_{n}$ at $x$.

A simple local computation shows that the first fundamental form of $S_{n}^{\star}$ coincides with the third fundamental form of $S_{n}$ (through the natural map $S_{n} \rightarrow S_{n}^{\star}$ ). In particular $S_{n}^{\star}$ is a surface of constant curvature $-1 / \sinh ^{2}\left(\theta_{n} / 2\right)$. Barbot, Beguin and Zeghib [4] have shown that there is a time function (K-time) $\mathfrak{t}_{n}: M_{n}^{\star} \rightarrow(-\infty, 0)$ such that $\mathfrak{t}_{n}^{-1}(k)$ is the unique surface in $M_{n}^{\star}$ with constant curvature $k$.

Lemma 6.4 is a simple consequence of Equation (32) and the following general lemma of de Sitter geometry.

Lemma 6.5 $[5 ; 6]$ Let $M^{\star}$ be a de Sitter MGHC spacetime and $\partial \widetilde{M}^{\star}$ be the boundary of its universal covering. If $S^{\star}$ is a constant curvature surface in $M^{\star}$, there is a natural 1-Lipschitz equivariant map

$$
\widetilde{r}^{\star}: \widetilde{S}^{\star} \rightarrow \partial \widetilde{M}^{\star}
$$

such that $\tilde{r}^{\star}(\tilde{x}) \in I^{-}(\tilde{x}) \cap \partial \widetilde{M}^{\star}$.

In particular, we have

$$
\ell_{\partial M^{\star}}(\gamma) \leq \ell_{S^{\star}}(\gamma),
$$

where $\ell_{\partial M^{\star}}$ and $\ell_{S^{\star}}$ are the marked length spectra of $\partial M^{\star}$ and $S^{\star}$ respectively. 
Proposition 6.6 The hyperbolic metrics $g_{\partial M_{n}}$ converge to $h$.

Proof By Lemmas 6.3 and 6.4, we have that up to passing to a subsequence $M_{n} \rightarrow M_{\infty}$. In particular, we can concretely realize $M_{n} \cong\left(S \times[0,+\infty), g_{M_{n}}\right)$ in such a way that $g_{M_{n}}$ converges to a hyperbolic metric $g_{M_{\infty}}$ such that $\left(S \times[0,+\infty), g_{M_{\infty}}\right) \cong M_{\infty}$, where we have denoted by the same symbol the metric $g_{M_{n}}$ on $M_{n}$ and the corresponding metric on the model $S \times[0,+\infty)$.

By abuse of notation, we denote again by $r_{n}: S \times[0,+\infty) \rightarrow S \times\{0\}$ the retraction corresponding to the retraction of $M_{n}$ onto $\partial M_{n}$. Let $\bar{\sigma}_{n}:(S, h) \rightarrow\left(S \times[0,+\infty), g_{M_{n}}\right)$ be the embedding with first fundamental form $I_{S_{n}}=\cosh ^{2}\left(\theta_{n} / 2\right) h_{n}$ and third fundamental form $I I I_{S_{n}}=\sinh ^{2}\left(\theta_{n} / 2\right) h_{n}^{\star}$.

Notice that the composition $i_{n}=r_{n} \circ \bar{\sigma}_{n}:\left(S, \cosh ^{2}\left(\theta_{n} / 2\right) h_{n}\right) \rightarrow\left(S \times\{0\}, g_{\partial M_{n}}\right)$ is a 1 -Lipschitz homotopy equivalence. So $i_{n}$ converges (up to passing to a subsequence) to a $1-$ Lipschitz homotopy equivalence $i_{\infty}:(S, h) \rightarrow\left(S \times\{0\}, g_{\partial M_{\infty}}\right)$. Since both $h$ and $g_{\partial M_{\infty}}$ are hyperbolic metrics, we conclude that $i_{\infty}$ is an isometry.

Let $\lambda_{\infty}$ the bending lamination of $M_{\infty}$. In order to conclude the proof of Equation (31) we need to show that $\lambda_{\infty}=\lambda / 2$. In fact, the following general result in Lorentzian geometry and (32) show that

$$
\iota\left(\lambda_{\infty}, \gamma\right)=\lim _{n} \ell_{S_{n}^{\star}}(\gamma)=\lim _{n} \ell_{I I I_{S_{n}}}(\gamma)=\lim _{n} \frac{\theta_{n}}{2} \ell_{h_{n}^{\star}}(\gamma)=\iota(\lambda / 2, \gamma),
$$

for every closed curve $\gamma$.

Proposition $6.7[5 ; 6]$ Let $\left(X_{n}^{\star}\right)_{n \in \mathbb{N}}$ be a sequence of MGHC de Sitter (or antide Sitter) spacetimes homeomorphic to $S \times \mathbb{R}$. Suppose that $X_{n}^{\star}$ converges to a MGHC spacetime $X_{\infty}^{\star}$. Take any sequence of numbers $k_{n} \rightarrow-\infty$ and let $\Sigma_{n}^{\star}$ be the future-convex surface of constant curvature $k_{n}$ contained in $X_{n}^{\star}$.

Denote by $\ell_{0}$ the length spectrum of the initial singularity of $X_{\infty}^{\star}$. Then, for every $\gamma \in \pi_{1}(S)$ we have

$$
\ell_{\Sigma_{n}^{\star}}(\gamma) \rightarrow \ell_{0}(\gamma)
$$

as $n \rightarrow+\infty$.

In the next section we will give a short description of the initial singularity for anti-de Sitter spacetimes and we will apply Proposition 6.7 to this case. 
Remark 6.8 In [5], Proposition 6.7 is proved assuming slightly different hypotheses. In fact, assuming that $X^{\star}$ is a fixed flat space-time, it is proved that the length spectrum of the level set $\Sigma^{\star}(t)$ of the $K$-time monotonically converges to the length spectrum of the initial singularity:

$$
\ell_{\Sigma^{\star}(t)} \searrow \ell_{0} \quad \text { as } t \rightarrow-\infty
$$

By [3] it is not difficult to see that the $K$-time continuously depend on the geometric structure; that is, if $X_{n}^{\star}$ is a sequence of flat space-times converging to $X_{\infty}^{\star}$, then for $t$ fixed the length spectrum $\ell_{\Sigma_{n}^{\star}(t)}$ converges to the length spectrum $\ell_{\Sigma_{\infty}^{\star}(t)}$, and the length spectrum of the initial singularity of $X_{n}^{\star}$ converges to the length spectrum of $X_{\infty}$. Then, by the monotonicity of the convergence $\ell_{\Sigma_{n}^{\star}(t)} \searrow \ell_{n, 0}$, for any sequence $t_{n} \rightarrow-\infty$ one gets that $\ell_{\Sigma_{n}^{\star}\left(t_{n}\right)} \rightarrow \ell_{\infty, 0}$ as $n \rightarrow+\infty$.

In order to apply the same argument to the de Sitter and anti-de Sitter case, the key point is then to prove (34). In the de Sitter case, the argument used in [5] can be rephrased verbatim. In fact, it is based on the convexity of the $K$-time and the cosmological time which are true also in the de Sitter case.

In the anti-de Sitter case, the $K$-time is still convex, but the cosmological time is not. Thus the argument must be slightly adapted. The convexity of the $K$-time implies the length spectrum of $\Sigma^{\star}(t)$ is monotonically increasing and $\liminf _{t \rightarrow-\infty} \ell_{\Sigma^{\star}(t)} \geq \ell_{0}$. In order to prove that $\lim \sup _{t \rightarrow-\infty} \ell_{\Sigma^{\star}(t)} \leq \ell_{0}$, one can use that the universal covering of $\Sigma^{\star}(t)$ is the graph of a function $u_{t}$ in $\mathbb{A d}^{3} \cong \mathbb{H}^{2} \times S^{1}$ which converges to a function $u_{0}$ whose graph is the past boundary of $\tilde{X}^{\star}$. By the convexity, we have that also $\operatorname{grad}\left(u_{t}\right)$ converges to $\operatorname{grad}\left(u_{0}\right)$ almost everywhere. This fact can be used to prove that, given a loop $\gamma$ in $S$, every path $\widetilde{\zeta}$ joining $\tilde{x}$ to $\gamma \cdot \tilde{x}$ in $\partial_{-} \tilde{X}^{\star}$ can be approximated by paths $\tilde{\zeta}_{t}$ on $\tilde{\Sigma}^{\star}(t)$ whose endpoints are $\gamma$-related, so that $\ell\left(\widetilde{\zeta}_{t}\right) \rightarrow \ell\left(\widetilde{\zeta}_{0}\right)$. This implies that $\limsup _{t \rightarrow-\infty} \ell_{\Sigma^{\star}(t)} \leq \ell_{0}$.

\subsection{Convergence on the real axis}

In this section we will prove that

$$
L_{\theta_{n}}^{\prime 1}\left(h, h_{n}^{\star}\right) \rightarrow E_{\lambda / 2}(h) .
$$

Recall that $L_{\theta_{n}}^{\prime 1}$ is the composition of the map $L_{\theta_{n}}^{\prime}: \mathcal{T} \times \mathcal{T} \rightarrow \mathcal{T} \times \mathcal{T}$ with the projection on the first factor.

As in the previous section, we will need a slightly stronger statement. 
Proposition 6.9 Let $\left(h_{n}\right)_{n \in \mathbb{N}}$ and $\left(h_{n}^{\star}\right)_{n \in \mathbb{N}}$ be two sequences of hyperbolic metrics such that $\left(h_{n}\right)_{n \in \mathbb{N}}$ converges to a hyperbolic metric $h$ on $S$ and that $\left(h_{n}^{\star}\right)$ converges to a point $[\lambda]$ in Thurston boundary of $\mathcal{T}$. If $\theta_{n}$ is a sequence of positive numbers such that $\theta_{n} \ell_{h_{n}^{\star}} \rightarrow \iota(\lambda, \bullet)$, then $L_{\theta_{n}}^{\prime 1}\left(h_{n}, h_{n}^{\star}\right)$ converges to $E_{\lambda / 2}(h)$.

Recall that the holonomy of $L_{\theta_{n}}^{\prime 1}\left(h_{n}, h_{n}^{\star}\right)$ corresponds to the left holonomy of the MGHC AdS manifold $N_{n} \cong S \times \mathbb{R}$ containing a future-convex $K$-surface $F_{n}$ with $I_{F_{n}}=\cos ^{2}\left(\theta_{n} / 2\right) h_{n}$ and $I I I_{F_{n}}=\sin ^{2}\left(\theta_{n} / 2\right) h_{n}^{\star}$. In order to prove Proposition 6.9, we will show that $N_{n}$ converges to a MGHC AdS structure $N_{\infty}$ and $F_{n}$ converges to the lower boundary $\partial_{-} C\left(N_{\infty}\right)$ of the convex core of $N_{\infty}$. Then we will prove that $\partial_{-} C\left(N_{\infty}\right)$ is isometric to $(S, h)$ and is bent along a lamination corresponding to $\lambda / 2$. By a result of Mess [29], the left holonomy of $N_{\infty}$ (that is, by definition, the limit of the left holonomies of $N_{n}$ ) is equal to the holonomy of $E_{\lambda / 2}(h)$, and Proposition 6.9 follows.

In order to prove that $N_{n}$ converges to some structure, we will consider the lifting $\phi_{n}: \widetilde{S} \rightarrow \tilde{N}_{n} \subset \mathbb{A d S}^{3}$ corresponding to the embedding $\bar{\phi}_{n}: S \rightarrow F_{n} \subset N_{n}$. The map $\phi_{n}$ is determined up to isometry of $\mathbb{A d S}^{3}$ and we will normalize it by requiring that, for some fixed $\tilde{p}_{0} \in \tilde{S}, \phi_{n}\left(\tilde{p}_{0}\right)=\tilde{x}_{0}$ and the normal vector to $\widetilde{F}_{n}=\phi_{n}(\tilde{S})$ at $\tilde{x}_{0}$ is equal to $\tilde{v}_{0}$ for some fixed $\tilde{x}_{0}, \widetilde{v}_{0}$ in $\mathbb{A d S}^{3}$.

The first step to prove the convergence of $N_{n}$ is to show that $\phi_{n}$ converges to a spacelike embedding into $\mathbb{A} \mathbb{d}^{3}$.

Proposition 6.10 Up to passing to a subsequence, $\widetilde{F}_{n}$ converges to a spacelike surface $\widetilde{F}_{\infty}$ in $\mathbb{A d S}^{3}$ and the map $\phi_{n}$ converges to an embedding

$$
\phi_{\infty}: \widetilde{S} \rightarrow \mathbb{A d}^{3}
$$

whose image is $\widetilde{F}_{\infty}$.

The easy part of the proof is to show that $\widetilde{F}_{n}$ converges to an embedded surface $\widetilde{F}_{\infty}$ in $\mathbb{A d S}^{3}$ that is achronal. The main issue is to show that the surface $\widetilde{F}_{\infty}$ is spacelike. The proof relies on the fact that, for some fixed $\tilde{p} \in \widetilde{S}$, the tangent planes of $\widetilde{F}_{n}$ at $\phi_{n}(\tilde{p})$ are uniformly spacelike, in the sense that they cannot approximate lightlike planes.

The proof of this fact is based on the technical Lemma 6.11.

Lemma 6.11 Let $b_{n}$ be the $h_{n}$-self adjoint operator such that $h_{n}^{\star}=h_{n}\left(b_{n} \bullet, b_{n} \bullet\right)$ and let $\bar{\sigma}_{n}: S \rightarrow \underset{\widetilde{S}}{S_{n} \subset M_{n}}$ be the embedding introduced in Section 6.1. Denote by $I_{\tilde{S}_{n}}^{\#}$ the lifting to $\widetilde{S}$ of the grafted metric $I_{S_{n}}^{\#}$ introduced in Definition 2.1. Then, for every compact set $K \subset \widetilde{S}$, there is a constant $C_{K}$ such that the diameter of $K$ with respect to $I_{S_{n}}^{\#}$ is bounded by $C_{K}$ for every $n$. 
Proof For any $k \in[-1,0), M_{n}$ contains exactly one $K$-surface of constant curvature $k$, denoted here by $M_{n}(k)$ (where by $M_{n}(-1)$ we mean the boundary of $M_{n}$ ). For each $n$, let $G_{n}=S G r_{\theta_{n}}^{\prime}\left(h_{n}, h_{n}^{\star}\right)$ be the projective surface at infinity of $M_{n}$. Let us consider the natural retraction $\Pi_{M_{n}(k)}: G_{n} \rightarrow M_{n}(k)$, which is the limit of the closest point projections $M_{n}(K) \rightarrow M_{n}(k)$ onto the convex surface $M_{n}(k)$ as $K>k$ converges to 0 (see Figure 1).

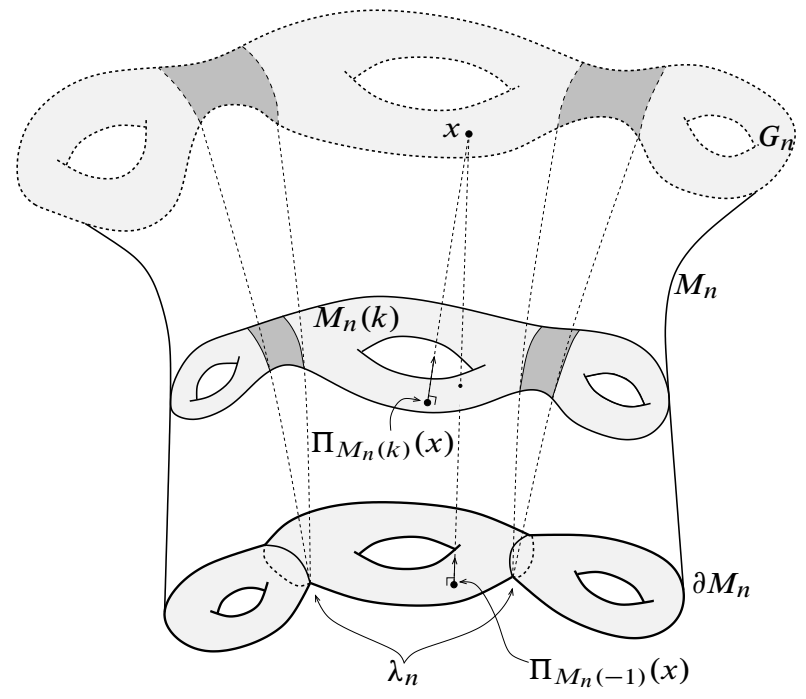

Figure 1: The retraction $\Pi_{M_{n}(k)}$

On the universal covering, $\Pi_{\widetilde{M}_{n}(k)}$ sends a point $\tilde{x} \in \widetilde{G}_{n}$ to the tangency point of the unique horocycle centered at $\widetilde{x}$ and tangent to $\widetilde{M}_{n}(k)$. For $k \in(-1,0)$, the map $\Pi_{M_{n}(k)}$ is a diffeomorphism and the inverse is the map obtained by sending each point of $y \in M_{n}(k)$ to the final point of the geodesic ray starting from $y$ and orthogonal to $M_{n}(k)$. If $k=-1$, the projection $\Pi_{M_{n}(-1)}$ is not injective in general, since points on $M_{n}(-1)$ can admit several normal directions. Nevertheless, $\Pi_{M_{n}(-1)}: G_{n} \rightarrow M_{n}(-1)$ is a homotopy equivalence.

In [37], it has been shown that, for $k \in(-1,0)$, the diffeomorphism $\Pi_{M_{n}(k)}$ is conformal with respect to the grafted metric $I_{M_{n}(k)}^{\#}$ of $M_{n}(k)$. The conformal factor is an increasing function of $k$ : this precisely means that the conformal map

$$
\Pi_{M_{n}\left(k^{\prime}\right)} \circ \Pi_{M_{n}(k)}^{-1}:\left(M_{n}(k), I_{M_{n}(k)}^{\#}\right) \rightarrow\left(M_{n}\left(k^{\prime}\right), I_{M_{n}\left(k^{\prime}\right)}^{\#}\right)
$$

decreases the lengths when $k>k^{\prime}$. 
Now notice that $S_{n}$ is equal to $M_{n}\left(K_{n}\right)$ for $K_{n}=-1 / \cosh ^{2}\left(\theta_{n}\right)$. As definitively $K_{n}<-1 / 2$, the map

$$
j_{n}=\bar{\sigma}_{n}^{-1} \circ \Pi_{S_{n}} \circ \Pi_{M_{n}(-1 / 2)}^{-1}:\left(M_{n}(-1 / 2), I_{M_{n}(-1 / 2)}^{\#}\right) \rightarrow\left(S, I_{S_{n}}^{\#}\right)
$$

decreases the lengths.

Since $M_{n}$ converges to an hyperbolic end $M_{\infty}$, the surface $M_{n}(-1 / 2)$ converges to $M_{\infty}(-1 / 2)$ in $C^{\infty}$-sense. This means that $M_{n}$ can be concretely realized as a hyperbolic metric $g_{M_{n}}$ on $S \times[0,+\infty)$ such that $M_{n}(-1 / 2)=S \times\{1\}$ and such that $g_{M_{n}}$ converges to a hyperbolic metric $g_{M_{\infty}}$ and $M_{\infty}(-1 / 2)=S \times\{1\}$. Then the family of $1-$ Lipschitz maps $j_{n}$ converges to the map $j_{\infty}=\bar{\sigma}_{\infty}^{-1} \circ \Pi_{S_{\infty}} \circ$ $\Pi_{M_{\infty}(-1 / 2)}^{-1}: M_{\infty}(-1 / 2) \rightarrow S$, which is a homotopy equivalence.

Let $\tilde{J}_{\infty}$ and $\tilde{J}_{n}$ be the lifting of those maps to the universal covering. Notice that $\tilde{J}_{\infty}$ is a proper map. If $K$ is a compact subset of $\widetilde{S}$, then $K^{\prime}=\tilde{J}_{\infty}^{-1}(K)$ is a compact subset of $\tilde{S} \times\{1\}$, and $K_{n}^{\prime}=\widetilde{J}_{n}^{-1}(K)$ is contained in some compact neighborhood of $K^{\prime}$ for every $n$. In particular, there exists a constant $C_{K}^{\prime}$ such that the diameter of $K_{n}^{\prime}$ with respect to $\widetilde{I}_{M_{n}(-1 / 2)}^{\#}$ is bounded by $C_{K}^{\prime}$ for all $n$. Taking $C_{K}$ bigger than $C_{K}^{\prime}$, it follows that the diameter of every $K_{n}^{\prime}$ with respect to $\widetilde{I}_{M_{n}(-1 / 2)}^{\#}$ is bounded by $C_{K}$. Since $\tilde{J}_{n}$ decreases the lengths, we have that the diameter of $K$ with respect to $I_{\widetilde{S}_{n}}^{\#}$ is bounded by $C_{K}$ for $n$ large enough.

Lemma 6.12 For every $d>0$ there is a compact set $K$ in $\mathbb{A d}^{3}$ such that for $\tilde{p} \in \widetilde{S}$ with $d_{h}\left(\tilde{p}, \tilde{p}_{0}\right)<d$, the normal vector $\widetilde{v}_{n}(\tilde{p})$ of $\widetilde{F}_{n}$ at $\phi_{n}(\tilde{p})$ lies in $K$.

Proof Lemma 6.11 implies that for any $d>0$, there is $D>0$ such that for any $n$ and any $\tilde{p} \in B_{\widetilde{h}}\left(\tilde{p}_{0}, d\right)$ there exists a path $\widetilde{\zeta}:[0,1] \rightarrow \widetilde{S}$ connecting $\tilde{p}_{0}$ to $\tilde{p}$ such that $\ell_{I_{\widetilde{S}_{n}}^{\#}}(\widetilde{\zeta})$ is bounded by $D$.

We claim (and will prove below) that this implies that

$$
\begin{aligned}
& \left|\left\langle\widetilde{x}_{0}, \widetilde{v}_{n}(\widetilde{p})\right\rangle\right| \leq 2 e^{2 D}, \\
& \left|\left\langle\widetilde{v}_{0}, \widetilde{v}_{n}(\tilde{p})\right\rangle\right| \leq 2 e^{2 D} .
\end{aligned}
$$

It follows from this claim that $\widetilde{v}_{n}(\tilde{p})$ is contained in

$$
K=\left\{w \in \mathbb{A d S}^{3} \mid\left\langle\widetilde{x}_{0}, w\right\rangle \leq 2 e^{2 D},\left\langle\widetilde{v}_{0}, w\right\rangle \leq 2 e^{2 D}\right\},
$$

which is a compact subset of $\mathbb{A d S}^{3}$, and the lemma follows. We now turn to the proof of the claim. 
We fix $n$ and consider the following functions:

$$
\begin{aligned}
a(t) & =-\left\langle\widetilde{x}_{0}, \phi_{n}(\widetilde{\varsigma}(t))\right\rangle, a_{\perp}(t)=-\left\langle\widetilde{x}_{0}, \widetilde{v}_{n}(\widetilde{\zeta}(t))\right\rangle, \\
a^{\star}(t) & =-\left\langle\widetilde{v}_{0}, \phi_{n}(\widetilde{\zeta}(t))\right\rangle, a_{\perp}^{\star}(t)=-\left\langle\widetilde{v}_{0}, \widetilde{v}_{n}(\widetilde{\zeta}(t))\right\rangle .
\end{aligned}
$$

Notice that $a$ is a positive function since $\tilde{x}_{0}$ and $\phi_{n}(\widetilde{\zeta}(t))$ are contained in a spacelike surface. Moreover, since the surface $\widetilde{F}_{n}$ is convex, the plane orthogonal to $\widetilde{v}_{n}(\widetilde{\varsigma}(t))$ is a support plane for $\widetilde{F}_{n}$, so it is not difficult to check that also $a_{\perp}$ is positive (see Figure 2).

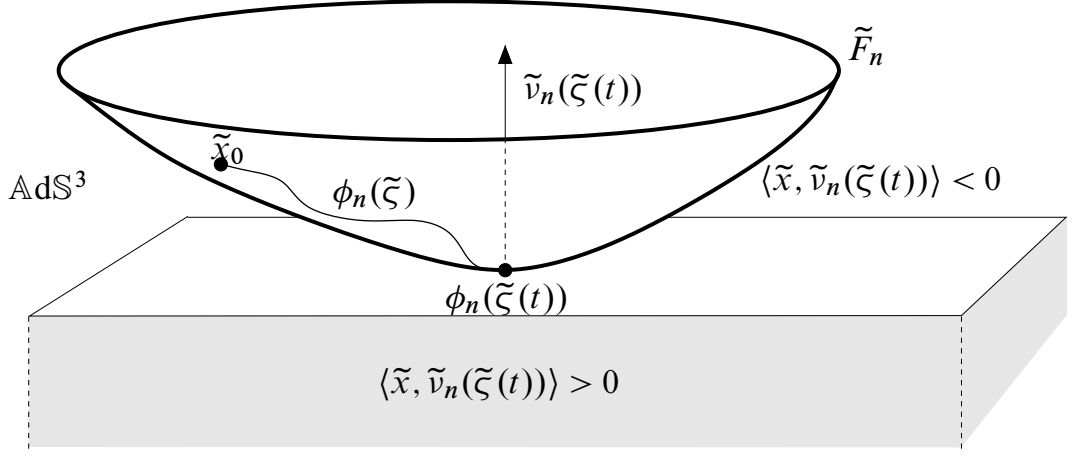

Figure 2: The product $\left\langle\widetilde{x}_{0}, \widetilde{v}_{n}(\widetilde{\zeta}(t))\right\rangle$ is negative.

We can decompose $\tilde{x}_{0}$ as

$$
\tilde{x}_{0}=a(t) \tilde{\phi}_{n}(\widetilde{\zeta}(t))+a_{\perp}(t) \widetilde{v}_{n}(\widetilde{\zeta}(t))+\widetilde{v}(t),
$$

with $\widetilde{v}(t) \in T_{\phi_{n}(\widetilde{\varsigma}(t))} \widetilde{F}_{n}$. Imposing $\left\langle\widetilde{x}_{0}, \widetilde{x}_{0}\right\rangle=-1$, we deduce that $\|\widetilde{v}(t)\| \leq a+a_{\perp}$. On the other hand, $\|\widetilde{v}(t)\| \leq\|\widetilde{v}(t)\|_{\tilde{h}_{n}}$ and so

$$
\begin{aligned}
\dot{a} & =\left\langle\widetilde{x}_{0}, \dot{\phi}_{n} \circ \widetilde{\zeta}\right\rangle=\left\langle\tilde{v}, d \phi_{n}(\dot{\tilde{\zeta}})\right\rangle \leq\left(a+a_{\perp}\right)\|\dot{\tilde{\widetilde{\zeta}}}\|_{\widetilde{h}_{n}}, \\
\dot{a}_{\perp} & =\left\langle\widetilde{x}_{0}, B_{\widetilde{F}_{n}}\left(d \phi_{n}(\dot{\tilde{\zeta}})\right)\right\rangle \leq\left(a+a_{\perp}\right)\left\|B_{\widetilde{F}_{n}}(\dot{\tilde{\zeta}})\right\|_{\widetilde{h}_{n}} \leq\left(a+a_{\perp}\right) \frac{\theta_{n}}{2}\|\dot{\tilde{\zeta}}\|_{\tilde{h}_{n}^{\star}} .
\end{aligned}
$$

Since $I_{S_{n}}^{\#}$ dominates both $\tilde{h}_{n}$ and $\frac{\theta_{n}^{2}}{4} \tilde{h}_{n}^{\star}$ we see that

$$
\begin{aligned}
\left(a+a_{\perp}\right)(0) & =2, \\
\dot{a}+\dot{a}_{\perp} & \leq 2\left(a+a_{\perp}\right)\|\dot{\tilde{\zeta}}\|_{I_{\widetilde{S}_{n}}^{\#}},
\end{aligned}
$$

and by a simple integration we have $a+a_{\perp} \leq 2 e^{2 D}$. 
A similar argument can be applied to $a^{\star}$ and $a_{\perp}^{\star}$ using the fact that the path $\widetilde{v}_{n}(\widetilde{\zeta}(t))$ is contained in the dual surface $\widetilde{F}_{n}^{\star}$, that is the surface made of normal vectors of $\widetilde{F}_{n}$. Indeed, there is a natural map $\phi_{n}^{\star}: \widetilde{S} \rightarrow \widetilde{F}_{n}^{\star}$ that sends a point $\tilde{p}$ to the point dual to the plane tangent to $\widetilde{F}_{n}$ at $\phi_{n}(\widetilde{p})$. The corresponding embedding data are

$$
I_{\widetilde{F}_{n}^{\star}}=I I I_{\widetilde{F}_{n}}=\sin ^{2}\left(\theta_{n} / 2\right) h_{n}^{\star}, \quad B_{\widetilde{F}_{n}^{\star}}=-1 / \tan \left(\theta_{n} / 2\right) b_{n}^{-1} .
$$

In particular, $F_{n}^{\star}$ is a past-convex spacelike surface, and the previous argument shows that $a^{\star}+a_{\perp}^{\star} \leq 2 e^{2 D}$.

Proof of Proposition 6.10 We will consider the product model of $\mathbb{A d S}^{3}=\mathbb{H}^{2} \times S^{1}$, where the metric at some point $\left(\xi, e^{i \vartheta}\right)$ is

$$
g_{\mathbb{A d S}^{3}}=g_{\mathbb{H}^{2}}-\chi(\xi) d \vartheta
$$

where $\chi(\xi)=\cosh d_{\mathbb{H}^{2}}\left(\xi, \xi_{0}\right)$, where $\xi_{0}$ is some fixed point (see [9]).

By a lemma of Mess [29], the image of $\phi_{n}$ is the graph of some function $\mathbb{H}^{2} \ni \xi \mapsto$ $e^{i s_{n}(\xi)} \in S^{1}$ that satisfies the spacelike condition

$$
\left\|\operatorname{grad}\left(s_{n}\right)\right\|<1 / \chi
$$

We can also suppose that $\phi_{n}\left(\tilde{p}_{0}\right)$ is the point $\tilde{x}_{0}=\left(\xi_{0}, 0\right)$ and the normal vector of $\widetilde{F}_{n}$ at $\tilde{x}_{0}$ is the unit vertical vector.

By (39), the functions $s_{n}$ are uniformly Lipschitz on compact sets of $\mathbb{H}^{2}$. So, up to subsequence, $\widetilde{F}_{n}$ converges to a surface $\widetilde{F}_{\infty}$ which is the graph of some limit function $s_{\infty}$, that verifies $\left\|\operatorname{grad}\left(s_{\infty}\right)\right\| \leq 1 / \chi$ almost everywhere.

In order to show that $\widetilde{F}_{\infty}$ is spacelike, we need to prove that $s_{\infty}$ verifies the strict inequality (39) almost everywhere. Notice that the projection $\pi_{n}: \widetilde{F}_{n} \rightarrow \mathbb{H}^{2}$ increases the length, so the disk $D$ in $\mathbb{H}^{2}$ with center $\left(\xi_{0}, 0\right)$ and radius $r$ is contained in $\pi_{n} \circ \phi_{n}\left(B_{\widetilde{F}_{n}}\left(\widetilde{x}_{0}, r\right)\right)$. By Lemma 6.12 , the normal vectors of $\widetilde{F}_{n}$ on the cylinder based on $D$ are contained in some compact subset $K$ (independent of $n$ ).

Since the normal vector at $\left(\xi, s_{n}(\xi)\right)$ is the vector

$$
\frac{1}{\sqrt{1-\chi^{2}\left\|\operatorname{grad}\left(s_{n}\right)\right\|^{2}}}\left(\operatorname{grad}\left(s_{n}\right)+\frac{\partial}{\partial \vartheta}\right)
$$

under the natural identification $T\left(\mathbb{H}^{2} \times S^{1}\right)=T \mathbb{H}^{2} \oplus T S^{1}$, we deduce that there exists $\epsilon$ depending on $K$, such that

$$
\left\|\operatorname{grad}\left(s_{n}\right)\right\| \leq(1-\epsilon) / \chi
$$

for every $\xi \in D$ and every $n$. This shows that $\tilde{F}_{\infty}$ is spacelike. 
Moreover, the restriction of the projection $\pi_{n} \circ \phi_{n}:(\tilde{S}, h) \rightarrow \mathbb{H}^{2}$ on $B_{\tilde{h}}\left(\tilde{p}_{0}, r\right)$ is $C$ Lipschitz, for some constant $C$ depending only on $r$. Indeed, given a vector $\widetilde{v} \in T_{\tilde{p}} \widetilde{S}$, let $\widetilde{v}_{n}=d \phi_{n}(v)$ and $\tilde{u}_{n}=d \pi_{n}\left(\widetilde{v}_{n}\right)$. We have that $\tilde{v}_{n}=\tilde{u}_{n}+\left\langle\operatorname{grad}\left(s_{n}\right), \tilde{u}_{n}\right\rangle \partial / \partial \vartheta$, so $\cos ^{2}\left(\theta_{n} / 2\right) \tilde{h}_{n}(\widetilde{v}, \widetilde{v})=\left\langle\widetilde{v}_{n}, \widetilde{v}_{n}\right\rangle \geq\left\|\widetilde{u}_{n}\right\|^{2}-\chi^{2}\left\|\operatorname{grad}\left(s_{n}\right)\right\|^{2}\left\|\tilde{u}_{n}\right\|^{2} \geq \epsilon\left\|\tilde{u}_{n}\right\|^{2}$.

Since $h_{n} \rightarrow h$, there exists $C^{\prime}$ such that the identity map between $(S, h)$ and $\left(S, h_{n}\right)$ is $C^{\prime}$-Lipschitz for every $n$. It follows, after taking a subsequence, that $\left(\pi_{n} \circ \phi_{n}\right)$ converges to a map $\pi_{\infty}^{\prime}: \widetilde{S} \rightarrow \mathbb{H}^{2}$, so then we have that $\left(\phi_{n}\right)$ converges to the map $\phi_{\infty}(\tilde{p})=\left(\pi_{\infty}^{\prime}(\tilde{p}), s_{\infty}\left(\pi_{\infty}^{\prime}(\tilde{p})\right)\right.$.

We can prove now that the holonomy $\rho_{n}: \pi_{1}(S) \rightarrow \operatorname{Isom}_{0}\left(\mathbb{A d S}^{3}\right)$ of $N_{n}$ converges to a limit representation $\rho_{\infty}$ for which $\phi_{\infty}$ equivariant.

Lemma 6.13 If $\phi_{n}$ converges to a space-like embedding $\phi_{\infty}$, then the representation $\rho_{n}$ converges to a representation $\rho_{\infty}: \pi_{1}(S) \rightarrow \operatorname{Isom}_{0}\left(\mathbb{A d S}^{3}\right)$ such that $\widetilde{F}_{\infty}$ is $\rho_{\infty}$-equivariant.

Moreover, the left and right components of $\rho_{\infty}$ are discrete and faithful representations of $\pi_{1}(S)$ into $\mathrm{PSL}_{2}(\mathbb{R})$.

Proof First we prove that, for every $\gamma \in \pi_{1}(S)$, the sequence $\rho_{n}(\gamma)$ is bounded in $\operatorname{Isom}_{0}\left(\mathbb{A d S} \mathbb{S}^{3}\right)$.

Recall that we are assuming $\phi_{n}\left(\tilde{p}_{0}\right)=\tilde{x}_{0}$ for all $n$ and the normal vectors $\tilde{v}_{n}\left(\tilde{p}_{0}\right)$ are equal to $\tilde{v}_{0}$. Now the $\rho_{n}(\gamma)\left(\tilde{x}_{0}\right)=\phi_{n}\left(\gamma \tilde{p}_{0}\right)$ form a sequence converging to $\bar{x}_{0}=\phi_{\infty}\left(\gamma \tilde{p}_{0}\right)$ and the $\rho_{n}(\gamma)\left(\widetilde{v}_{0}\right)$ converge to a unit timelike vector $\bar{v}_{0}$ at $\bar{x}_{0}$ orthogonal to some support plane of $\widetilde{F}_{\infty}$.

This implies there is a bounded sequence of isometry of $\mathbb{A d S}^{3}$, says $\tau_{n}$, such that

$$
\tau_{n} \rho_{n}(\gamma)\left(\tilde{x}_{0}\right)=\tilde{x}_{0}, \quad \tau_{n} \rho_{n}(\gamma)\left(\widetilde{v}_{0}\right)=\tilde{v}_{0} .
$$

Now the set of isometries that fix $\tilde{x}_{0}$ and $\widetilde{v}_{0}$ is compact; so, after taking a subsequence, $\tau_{n} \rho_{n}(\gamma) \rightarrow \bar{\tau}$. Since up to passing to a subsequence we also have $\tau_{n} \rightarrow \tau_{\infty}$, we can deduce that $\rho_{n}(\gamma) \rightarrow \tau_{\infty}^{-1} \circ \bar{\tau}$.

To prove that $\rho_{n}$ is converging, it is sufficient to check that two converging subsequences of $\rho_{n}$ share the same limit. On the other hand, suppose that $\rho_{\infty}$ is a limit of a subsequence of $\rho_{n}$, then $\rho_{\infty}$ makes $\phi_{\infty}$ equivariant:

$$
\phi_{\infty}(\gamma \tilde{p})=\rho_{\infty}(\gamma) \phi_{\infty}(\tilde{p}) .
$$

This relation uniquely determines the action of $\rho_{\infty}(\gamma)$ on $\widetilde{F}_{\infty}$. Since two isometries of $\mathbb{A d S}^{3}$ that coincide on a spacelike surface are equal the result follows. 
The fact that the left and right representations $\pi_{1}(S) \rightarrow \mathrm{PSL}_{2}(\mathbb{R})$ corresponding to $\rho_{\infty}$ are faithful and discrete is a consequence of the fact that they are limit of faithful and discrete representations.

If $\left(\phi_{n_{i}}\right)$ is a convergent subsequence of $\left(\phi_{n}\right)$, then Lemma 6.13 implies that $N_{n_{i}}$ is a convergent sequence of spacetime. Let $N_{\infty}$ be the limit of such spacetimes. Its holonomy is by definition the limit of the holonomies of the $N_{n}$. In particular, we can concretely realize $N_{n}$ as an AdS metric $g_{N_{n}}$ on $S \times \mathbb{R}$, in such a way that $g_{N_{n_{i}}}$ converges to an AdS metric $g_{N_{\infty}}$ as tensors on $S \times \mathbb{R}$ and $\left(S \times \mathbb{R}, g_{N_{\infty}}\right) \cong N_{\infty}$.

Proposition 6.14 $F_{n_{i}}$ converges to the lower boundary $\partial_{-} C\left(N_{\infty}\right)$ of the convex core of $N_{\infty}$. Moreover, the induced map

$$
\bar{\phi}_{\infty}:(S, h) \rightarrow \partial_{-} C\left(N_{\infty}\right)
$$

is an isometry.

The proof of this proposition is based on the following lemma.

Lemma 6.15 If $N$ is a MGHC anti-de Sitter spacetime and $N(k)$ is a Cauchy surface of constant curvature $k \leq-1$, then the Lorentzian distance of any point of $N(k)$ from the convex core of $N$ is smaller than $\arctan \sqrt{|1+k|}$.

Proof We consider the point $x_{0}$ on $N(k)$ with the biggest distance from the convex core. If $d$ is the distance between $x_{0}$ and the convex core, then it is well known that $d<\pi / 2$ and there exists a timelike geodesic segment $\varsigma$ joining the point $x_{0}$ to a point $y_{0}$ on the boundary of the convex core with length equal to $d$ [7].

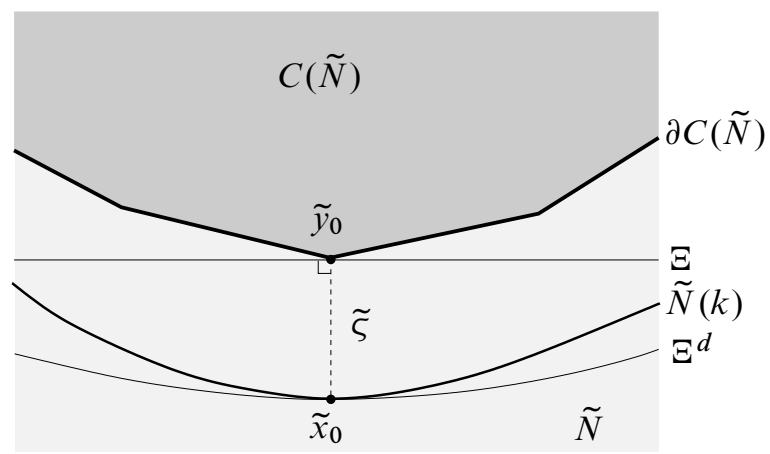

Figure 3: Estimating the distance between $\widetilde{N}(k)$ and $C(\tilde{N})$ 
We consider now a lift $\tilde{\zeta}$ of $\zeta$ to the universal cover $\tilde{N} \subset \mathbb{A d S}^{3}$ (see Figure 3). The plane $\Xi$ through $\tilde{y}_{0}$ orthogonal to $\tilde{\zeta}$ is a support plane for the lifting of the convex core. Let $\Xi^{d}$ be the surface of points in $\mathbb{A d S}^{3}$ whose distance from $\Xi$ is $d$. It is a convex surface of constant curvature $-1 / \cos ^{2}(d)$. Clearly, $\tilde{x}_{0}$ lies on $\Xi^{d}$ and $\tilde{N}(k)$ is contained in the convex side bounded by $\Xi^{d}$. In particular, $\tilde{N}(k)$ and $\Xi^{d}$ are tangent at $\tilde{x}_{0}$ and, by the maximum principle, the curvature of $\tilde{N}(k)$ is less than the curvature of $\Xi^{d}$ at $\tilde{x}_{0}$.

We deduce that $k \leq-1 / \cos ^{2}(d)$, which implies that $\tan (d) \leq \sqrt{|1+k|}$.

Proof of Proposition 6.14 Since the metrics $g_{N_{n_{i}}}$ converge to $g_{N_{\infty}}$, the convex cores of the $N_{n_{i}}$ converge to the convex core of $N_{\infty}$. (Since the metrics converge, the holonomy representations converge and so that their limit set in $\partial_{\infty} \mathbb{A d S}^{3}$ converge; therefore also their convex hulls, so the convex cores converge.)

In particular, the lower boundary $\partial_{-} C\left(N_{n_{i}}\right)$ of the convex core of $N_{n_{i}}$ converges to $\partial_{-} C\left(N_{\infty}\right)$. By Lemma 6.15 , the distance of any point of $F_{n_{i}}$ from $\partial_{-} C\left(N_{n_{i}}\right)$ is smaller that $\theta_{n_{i}} / 2$. This implies that $F_{n_{i}}$ converges to $\partial_{-} C\left(N_{\infty}\right)$.

In order to prove that the map $\bar{\phi}_{\infty}:(S, h) \rightarrow \partial_{-} C\left(N_{\infty}\right)$ is an isometry, it is sufficient to show that $\bar{\phi}_{\infty}$ increases the distances. Indeed, both $(S, h)$ and $\partial_{-} C\left(N_{\infty}\right)$ are hyperbolic surfaces and $\bar{\phi}_{\infty}$ is an homotopy equivalence.

We will prove that the lifting $\phi_{\infty}:(\tilde{S}, h) \rightarrow \partial_{-} C\left(\tilde{N}_{\infty}\right)$ increases the lengths. Given $\tilde{p}, \tilde{q} \in \tilde{S}$, we consider any path $\tilde{\zeta}:[0,1] \rightarrow \widetilde{S}$ connecting $\tilde{p}$ and $\widetilde{q}$ such that

- $\partial_{-} C\left(\tilde{N}_{\infty}\right)$ is smooth at almost all points of $\tilde{\varsigma}_{\infty}:=\phi_{\infty} \circ \tilde{\varsigma}$,

- $\ell\left(\tilde{\zeta}_{\infty}\right) \leq d_{\infty}\left(\tilde{x}_{\infty}, \tilde{y}_{\infty}\right)+\epsilon$,

where $\tilde{x}_{\infty}=\phi_{\infty}(\tilde{p})$ and $\tilde{y}_{\infty}=\phi_{\infty}(\widetilde{q})$ and $d_{\infty}$ is the distance in $\partial_{-} C\left(\tilde{N}_{\infty}\right)$.

In the model $\mathbb{H}^{2} \times S^{1}$ of $\mathbb{A d} \mathbb{S}^{3}$, the surfaces $\widetilde{F}_{n}$ are graphs of functions $e^{i s_{n}}: \mathbb{H}^{2} \rightarrow S^{1}$ converging to $e^{i s_{\infty}}: \mathbb{H}^{2} \rightarrow S^{1}$ such that $\partial_{-} C\left(\tilde{N}_{\infty}\right)$ is the graph of $e^{i s_{\infty}}$.

We have $\tilde{\zeta}_{\infty}(t)=\left(\xi(t), e^{i s_{\infty}(\xi(t))}\right)$ with $\xi:[0,1] \rightarrow \mathbb{H}^{2}$ Lipschitz function. Take $\tilde{\zeta}_{n}(t)=\left(\xi(t), e^{i s_{n}(\xi(t))}\right)$. For any smooth point $\tilde{x}=\left(\xi, e^{i s_{\infty}(\xi)}\right)$ of $\partial_{-} C\left(\tilde{N}_{\infty}\right)$ we have $\operatorname{grad}\left(s_{n}\right)(\xi) \rightarrow \operatorname{grad}\left(s_{\infty}\right)(\xi)$. Indeed, by convexity, tangent planes of $\widetilde{F}_{n}$ converge to support planes of $\partial_{-} C\left(\tilde{N}_{\infty}\right)$.

By the Lebesgue Theorem we have

$$
\begin{aligned}
\ell\left(\widetilde{\varsigma}_{n}\right)=\int_{0}^{1} \sqrt{\|\dot{\xi}\|^{2}-\chi(\xi)\left\langle\dot{\xi}, \operatorname{grad}\left(s_{n}\right)\right\rangle^{2}} d t & \\
& \rightarrow \int_{0}^{1} \sqrt{\|\dot{\xi}\|^{2}-\chi(\xi)\left\langle\dot{\xi}, \operatorname{grad}\left(s_{\infty}\right)\right\rangle^{2}} d t=\ell\left(\tilde{\varsigma}_{\infty}\right),
\end{aligned}
$$


since $\sqrt{\|\dot{\xi}\|^{2}-\chi(\xi)\left\langle\dot{\xi}, \operatorname{grad}\left(s_{n}\right)\right\rangle^{2}}$ are all dominated by $\|\dot{\xi}\|$, which is an integrable function.

Since the endpoints of $\widetilde{\zeta}_{n}$ correspond to points $\tilde{x}_{n}=\varphi_{n}\left(\tilde{p}_{n}\right)$ and $\tilde{y}_{n}=\varphi_{n}\left(\widetilde{q}_{n}\right)$ with $\tilde{p}_{n} \rightarrow \tilde{p}$ and $\widetilde{q}_{n} \rightarrow \tilde{q}$, we deduce that $d_{\tilde{h}}(\tilde{p}, \widetilde{q}) \leq d_{\infty}\left(\tilde{x}_{\infty}, \tilde{y}_{\infty}\right)+\epsilon$. Since $\epsilon$ can be chosen arbitrarily small,

$$
d_{\tilde{h}}(\tilde{p}, \tilde{q}) \leq d_{\infty}\left(\tilde{x}_{\infty}, \tilde{y}_{\infty}\right)
$$

This completes the proof.

So far, we have shown that $N_{n}$ is contained in a compact subset of the space of MGHC AdS structures, and any convergent subsequence of $\bar{\phi}_{n}: S \rightarrow N_{n}$ converges to an isometric embedding $\bar{\phi}_{\infty}:(S, h) \rightarrow N_{\infty}$, whose image is the lower boundary $\partial_{-} C\left(N_{\infty}\right)$ of the convex core of $N_{\infty}$.

Let $\lambda_{\infty}$ be the bending lamination of $\partial_{-} C\left(N_{\infty}\right)$. We will prove that $\lambda_{\infty}=\lambda / 2$. Since the length spectrum of the third fundamental form $I I I_{F_{n}}$ converges to the intersection spectrum of $\lambda / 2$, it is sufficient to prove that it converges also to the intersection spectrum of the bending lamination of $\partial_{-} C\left(N_{\infty}\right)$.

Now, let $\widetilde{F}_{n}^{\star}$ be the surface dual to $\widetilde{F}_{n}$. Points of $\widetilde{F}_{n}^{\star}$ are dual to tangent planes of $\widetilde{F}_{n}$ and $\widetilde{F}_{n}^{\star}$ is a past-convex surface of constant curvature $-1 / \sin ^{2}\left(\theta_{n} / 2\right)$ as (38) shows.

Clearly, $\widetilde{F}_{n}^{\star}$ is invariant under the holonomy action of $\pi_{1}(S)$, so it is contained in $\tilde{N}_{n}^{\star}$ and its quotient is a Cauchy surface $F_{n}^{\star}$ of $\tilde{N}_{n}^{\star}$. By Equation (38), the length spectrum of $\widetilde{F}_{n}^{\star}$ is equal to the length spectrum of the third fundamental form $I I I_{F_{n}}$.

The boundary of the domain $\tilde{N}_{\infty}$ in $\mathbb{A d S}^{3}$ is the union of two disjoint achronal surfaces: the past and the future singularities of $\tilde{N}_{\infty}$, that are clearly invariant under the action of $\pi_{1}(S)$.

By Proposition 6.7, the length spectrum of $F_{n}^{\star}$ converges to the length spectrum of the action of $\pi_{1}(S)$ on the future singularity of $\tilde{N}_{\infty}^{\star}$ (notice indeed that since $F_{n}^{\star}$ is past-convex, in order to apply Proposition 6.7 we need to exchange the time orientation). On the other hand, by [7] the length spectrum of the future singularity of $\tilde{N}_{\infty}^{\star}$ coincides with the intersection spectrum of the bending lamination of the lower boundary of the convex core of $N_{\infty}$.

Combining these facts, we deduce that

$$
\ell_{\text {III }_{F_{n}}}(\gamma) \rightarrow \iota\left(\lambda_{\infty}, \gamma\right)
$$

so $\lambda_{\infty}=\lambda / 2$. 


\subsection{Asymptotic behavior of the measures $\operatorname{tr}\left(b_{n}\right) \omega_{h_{n}}$}

Let $\left(h_{n}, h_{n}^{\star}\right)_{n \in \mathbb{N}}$ be a sequence of normalized hyperbolic metrics on $S$ such that $h_{n}$ converges to $h$ and $h_{n}^{\star}$ converges to $[\lambda]$ in Thurston boundary of Teichmüller space, and denote by $b_{n}$ the operator associated to $\left(h_{n}, h_{n}^{\star}\right)$ provided by Corollary 1.5.

Moreover, let $\left(\theta_{n}\right)_{n \in \mathbb{N}}$ be a sequence such that $\theta_{n} \ell_{h_{n}^{\star}}$ converges to $\iota(\lambda, \bullet)$ in the sense of spectra of closed curves.

In this section we study the asymptotic behavior of $\operatorname{tr}\left(b_{n}\right)$ : roughly speaking, it concentrates around the $h$-geodesic representative of $\lambda$. Hence, we will always refer to $\lambda$ as to such an $h$-geodesic representative.

These results will turn useful in the proof of Theorem 6.1 and in Section 7.

Proposition 6.16 Let $V \subset S$ a closed subsurface such that $\partial V$ is smooth and does not intersect $\lambda$. Call $\lambda_{V}$ the $h$-geodesic sublamination $\lambda \cap V$. Then

$$
\theta_{n} \int_{V} \operatorname{tr}\left(b_{n}\right) \omega_{h_{n}} \rightarrow \ell_{h}\left(\lambda_{V}\right)
$$

where $\omega_{g}$ is the area form associated to $g$.

In order to prove Proposition 6.16, we need the following lemma that is analogous to Lemma 6.15.

Lemma 6.17 Let $M$ be a hyperbolic end associated to some projective structure on $S$, and let $M(k)$ be the surface of constant curvature $k$ with $k \in[-1,0)$. Then the distance of any point of $M(k)$ from the boundary of $M$ is at most $\operatorname{arctanh} \sqrt{1+k}$.

The proof of Lemma 6.17 is essentially the same as in Lemma 6.15. We leave the details to the reader.

Corollary 6.18 The family of isometric immersions $\sigma_{n}:\left(\tilde{S}, \cosh ^{2}\left(\theta_{n} / 2\right) \tilde{h}\right) \rightarrow \mathbb{H}^{3}$ converges to a bending map $\sigma_{\infty}:(\widetilde{S}, h) \rightarrow \mathbb{H}^{3}$, with bending lamination $\lambda / 2$.

Proof Since $\sigma_{n}$ are uniformly Lipschitz as maps $(\tilde{S}, \tilde{h}) \rightarrow \mathbb{H}^{3}$, they converge up to subsequences to a locally convex surface. Combining Proposition 6.2 and Lemma 6.17, we deduce that this surface is the bent surface corresponding to $\operatorname{Gr}_{\lambda / 2}(S)$.

Proof of Proposition 6.16 We consider the embedding $\bar{\sigma}_{n}: S \rightarrow S_{n} \in M_{n}$ inside a hyperbolic end $M_{n}$ such that

$$
I_{S_{n}}=\cosh ^{2}\left(\theta_{n} / 2\right) h_{n}, \quad B_{S_{n}}=\tanh \left(\theta_{n} / 2\right) b_{n} .
$$


The projective structure $G_{n}$ at the ideal boundary of $M_{n}$ converges to $G_{\infty}=G r_{\lambda / 2}(h)$ (Proposition 6.2). Call $M_{\infty}$ the hyperbolic end determined by the $\mathbb{C} \mathbb{P}^{1}$-surface $G_{\infty}$. A simple computation shows that the area element of $S_{n}$ with respect to $I_{S_{n}}^{\#}$ is $\omega_{I_{S_{n}}^{\#}}=\left(\cosh ^{2}\left(\theta_{n} / 2\right)+\sinh ^{2}\left(\theta_{n} / 2\right)+\sinh \left(\theta_{n} / 2\right) \cosh \left(\theta_{n} / 2\right) \operatorname{tr}\left(b_{n}\right)\right) \omega_{h_{n}}$ and so the area of $\bar{\sigma}_{n}(V)$ is

$$
\operatorname{Area}_{I_{S_{n}}^{\#}}(V)=\left(\operatorname{Area}_{h_{n}}(V)+\frac{\theta_{n}}{2} \int_{V} \operatorname{tr}\left(b_{n}\right) \omega_{h_{n}}\right)\left(1+o\left(\theta_{n}\right)\right) .
$$

As before, we can identify $M_{n} \cup G_{n} \cong\left(S \times[0, \infty), g_{M_{n}}\right) \cup S \times\{\infty\}$ so that

- the developing map $\operatorname{dev}_{n}: \tilde{S} \times[0, \infty] \rightarrow \overline{\mathbb{H}}^{3}$ converges to $\operatorname{dev}_{\infty}$ (and so we have $\left.g_{M_{n}} \rightarrow g_{M_{\infty}}\right)$,

- $\bar{\sigma}_{n}$ converges to the pleated surface $\bar{\sigma}_{\infty}: S \rightarrow\left(S \times\{0\}, g_{M_{\infty}}\right)$.

We will call $\partial^{d} M_{n}$ the surface in $M_{n}$ at distance $d$ from the boundary and let $\Pi_{\partial^{d} M_{n}}: G_{n} \rightarrow \partial^{d} M_{n}$ the projection introduced in Lemma 6.11 .

There exists two numbers $\epsilon_{n}<\delta_{n}$ such that $S_{n}$ is contained between $\partial^{\epsilon_{n}} M_{n}$ and $\partial^{\delta_{n}} M_{n}$ and, by Lemma $6.17, \delta_{n} \rightarrow 0$ as $n \rightarrow+\infty$.

By the monotonicity result proved in [37],

$$
\Pi_{\partial \epsilon n}^{*} M_{n}\left(I_{\partial \epsilon n}^{\#} M_{n}\right) \leq \Pi_{S_{n}}^{*}\left(I_{S_{n}}^{\#}\right) \leq \Pi_{\partial \delta^{\delta} M_{n}}^{*}\left(I_{\partial^{\delta} n}^{\#} M_{n}\right) .
$$

If $\lambda_{n}$ is the bending lamination of $M_{n}$, then the grafted metric on $\partial^{d} M_{n}$ makes it isometric to $e^{2 d} g_{G_{n}}$, where $g_{G_{n}}$ is Thurston metric on the projective surface $G_{n}=G r_{\lambda_{n}}\left(\partial M_{n}, g_{M_{n}}\right)$.

So we deduce that

$$
e^{2 \epsilon_{n}} \omega_{G_{n}} \leq \Pi_{S_{n}}^{*}\left(\omega_{I_{S_{n}}^{\#}}\right) \leq e^{2 \delta_{n}} \omega_{G_{n}}
$$

and so

$$
e^{2 \epsilon_{n}} \operatorname{Area}_{G_{n}}\left(\Pi_{S_{n}}^{-1}\left(\bar{\sigma}_{n}(V)\right)\right) \leq \operatorname{Area}_{I_{S_{n}}^{\#}}(V) \leq e^{2 \delta_{n}} \operatorname{Area}_{G_{n}}\left(\Pi_{S_{n}}^{-1}\left(\bar{\sigma}_{n}(V)\right)\right) .
$$

Since $G_{n} \rightarrow G_{\infty}$, their Thurston metrics converge to $g_{G_{\infty}}$. We claim that $\Pi_{S_{n}}^{-1}\left(\bar{\sigma}_{n}(V)\right)$ converge to $\Pi_{S_{\infty}}^{-1}\left(\bar{\sigma}_{\infty}(V)\right)$ in the Hausdorff sense, and so

$$
\operatorname{Area}_{I_{S_{n}}^{\#}}(V) \rightarrow \operatorname{Area}_{G_{\infty}}\left(\Pi_{S_{\infty}}^{-1}\left(\bar{\sigma}_{\infty}(V)\right)\right)=\operatorname{Area}_{h}(V)+\frac{1}{2} \ell_{h}\left(\lambda_{V}\right)
$$

by Equation (42).

The result will follow by comparing Equations (41) and (43).

In order to prove the claim, it is enough to prove that $\partial \Pi_{S_{n}}^{-1}\left(\bar{\sigma}_{n}(V)\right) \rightarrow \partial \Pi_{S_{\infty}}^{-1}\left(\bar{\sigma}_{\infty}(V)\right)$, which would follow from the fact that $\left.\Pi_{S_{n}}^{-1} \circ \bar{\sigma}_{n}\right|_{\partial V}$ converges to $\left.\Pi_{S_{\infty}}^{-1} \circ \bar{\sigma}_{\infty}\right|_{\partial V}$. 
Notice that $\Pi_{S_{n}}$ is a diffeomorphism, and $\left.\Pi_{S_{\infty}}^{-1} \circ \bar{\sigma}_{\infty}\right|_{\partial V}$ is well-defined and continuous since $\partial V$ does not intersect $\lambda$.

Let $\left(p_{n}\right)_{n \in \mathbb{N}}$ be a sequence of points in $\partial V$ such that $p_{n} \rightarrow p$. The point $\Pi_{S_{n}}^{-1}\left(\bar{\sigma}_{n}\left(p_{n}\right)\right)$ is the ideal point of the horocycle $U_{n}$ tangent to $S_{n}$ at $\bar{\sigma}_{n}\left(p_{n}\right)$. By convexity of the surfaces $S_{n}$, one can easily see that $U_{n}$ converges to a horocycle $U_{\infty}$ tangent to $S_{\infty}$ at $\bar{\sigma}_{\infty}(p)$. Since $p \notin \lambda$, such a $U_{\infty}$ is unique and so its ideal point is necessarily $\Pi_{S_{\infty}}^{-1}(p)$.

Corollary 6.19 Let $h_{n}$ be a sequence of hyperbolic metrics converging to $h$, and let $h_{n}^{\star}$ be a diverging sequence of metrics. Then, $\left(\theta_{n} \ell_{h_{n}^{\star}}(\gamma)\right)_{n \in \mathbb{N}}$ is bounded for every $\gamma \in \pi_{1}(S)$ if and only if $\theta_{n} \int_{S} \operatorname{tr}\left(b_{n}\right) \omega_{h_{n}}$ is bounded.

\subsection{Proof of Theorems 1.12 and 6.1}

Proof of Theorem 1.12 Let us fix a hyperbolic metric $h$ on $S$ and a sequence of hyperbolic metrics $h_{n}^{\star}$ converging to a point $[\lambda]$ in Thurston boundary of $\mathcal{T}$. Fixing a sequence $\theta_{n}$ such that $\theta_{n} \ell_{h_{n}^{\star}}$ converges to $\iota(\lambda, \bullet)$, let us set

$$
L_{e^{i \theta n}}\left(h, h_{n}^{\star}\right)=\left(h_{n}^{1}, h_{n}^{2}\right) .
$$

In Section 6.2 we showed $h_{n}^{1} \rightarrow E_{\lambda / 2}(h)$. To conclude the proof we need to prove

$$
\theta_{n} \ell_{h_{n}^{2}} \rightarrow \iota(\lambda, \bullet)
$$

The main issue to prove (44) is to show that for every $\gamma \in \pi_{1}(S)$, there is $C=C(\gamma)$ such that

$$
\theta_{n} \ell_{h_{n}^{2}}(\gamma)<C
$$

Corollary 6.19 indicates that, in order to prove (45), it is sufficient to bound

$$
\theta_{n} \int_{S} \operatorname{tr}\left(b_{n}^{\prime}\right) \omega_{h_{n}^{1}}
$$

where $b_{n}^{\prime}$ is the $h_{n}^{1}$-self-adjoint operator such that $h_{n}^{2}=h_{n}^{1}\left(b_{n}^{\prime} \bullet, b_{n}^{\prime} \bullet\right)$. Now we have that $b_{n}^{\prime}=\beta_{n}^{\prime} b_{n} \beta_{n}^{-1}$ where $\beta_{n}=\cos \left(\theta_{n} / 2\right) E+\sin \left(\theta_{n} / 2\right) J b_{n}$ and $\omega_{h_{n}^{1}}=\operatorname{det}\left(\beta_{n}\right) \omega_{h}=\omega_{h}$. So, $\theta_{n} \int_{S} \operatorname{tr}\left(b_{n}^{\prime}\right) \omega_{h_{n}^{1}}=\theta_{n} \int_{S} \operatorname{tr}\left(b_{n}\right) \omega_{h}$ that in turn is bounded since $\theta_{n} \ell_{h_{n}^{\star}}(\gamma)$ is bounded for every $\gamma \in \pi_{1}(S)$ by hypothesis.

It follows that there exists a measured geodesic lamination $\mu$ such that, up to passing to a subsequence, $\theta_{n} \ell_{h_{n}^{2}} \rightarrow \iota(\mu, \bullet)$. To show that $\mu=\lambda$, notice that by Proposition 6.9 we have that

$$
L_{e^{i \theta n}}^{1}\left(h_{n}^{1}, h_{n}^{2}\right) \rightarrow E_{\mu / 2}\left(h_{\infty}\right)
$$


On the other hand, we have that

$$
L_{e^{i \theta_{n}}}^{1}\left(h_{n}^{1}, h_{n}^{2}\right)=L_{e^{i \theta_{n}}}^{1}\left(L_{e^{i \theta_{n}}}\left(h, h_{n}^{\star}\right)\right)=L_{e^{2 i \theta_{n}}}^{1}\left(h, h_{n}^{\star}\right) .
$$

So, applying again Proposition 6.9, we obtain that

$$
L_{e^{i \theta n}}^{1}\left(h_{n}^{1}, h_{n}^{2}\right) \rightarrow E_{\lambda}(h)=E_{\lambda / 2}\left(h_{\infty}\right) .
$$

Comparing (46) and (47) we conclude that $E_{\lambda / 2}\left(h_{\infty}\right)=E_{\mu / 2}\left(h_{\infty}\right)$ and so $\lambda=\mu$.

Proof of Theorem 6.1 Let $h$ and $h_{n}$ as above. For all $z=t+i s \in \overline{\mathbb{H}}$, we have to prove

$$
P_{\theta_{n} z}^{\prime}\left(h, h_{n}^{\star}\right) \rightarrow G r_{s \lambda / 2}\left(E_{-t \lambda / 2}(h)\right) .
$$

Recall that $P_{\theta_{n} z}^{\prime}\left(h, h_{n}^{\star}\right)=S G r_{\theta_{n} s} \circ L_{-\theta_{n} t}^{\prime}\left(h, h_{n}^{\star}\right)$. Note that if we put

$$
L_{-\theta_{n} t}^{\prime}\left(h, h_{n}^{\star}\right)=\left(h_{n}^{1}, h_{n}^{2}\right),
$$

Theorem 1.12 shows that $h_{n}^{1} \rightarrow h_{\infty}=E_{-t \lambda / 2}(h)$ and $\theta_{n} \ell_{h_{n}^{2}} \rightarrow \lambda$.

Applying Proposition 6.2 we conclude that

$$
P_{\theta_{n} z}^{\prime}\left(h, h_{n}^{\star}\right)=S G r_{\theta_{n} s}\left(h_{n}^{1}, h_{n}^{2}\right) \rightarrow G r_{s \lambda / 2}\left(E_{-t \lambda / 2}(h)\right) .
$$

\subsection{Convergence of the distances}

The aim of this section is to study the asymptotic behavior of the sequence of distances induced by the metrics $\theta_{n}^{2} h_{n}^{\star}$ introduced in the previous section. By our assumption, the length spectrum of $h_{n}^{\star}$ rescaled by $\theta_{n}$ converges to the intersection with $\lambda$. Notice that this assumption only concerns the isotopy class of $h_{n}^{\star}$. On the other hand, once we concretely fix $h$, the metric $h_{n}^{\star}$ is uniquely determined in its isotopy class by requiring the identity map $(S, h) \rightarrow\left(S, h_{n}^{\star}\right)$ is minimal Lagrangian. The result we consider in this section deals with the asymptotic behavior of $h_{n}^{\star}$ considered as concrete metrics on $S$. Clearly, these results are valid for this choice of gauge, and are no longer valid if we change $h_{n}^{\star}$ by some isotopy.

Notice that the representative $\lambda$ of a point in Thurston boundary of $\mathcal{T}(S)$ can be chosen to be a measured geodesic lamination for any hyperbolic metric on $S$. In order to study the behavior of $h_{n}^{\star}$, it is natural to fix $\lambda$ as the concrete measured geodesic realization with respect to the metric $h$.

Let us fix a universal cover $\widetilde{S} \rightarrow \underset{\widetilde{\lambda}}{S}$ and denote by $\tilde{h}$ and $\widetilde{h}_{n}^{\star}$ the pullback of the metrics $h$ and $h_{n}^{\star}$ on $\widetilde{S}$. Finally let $\tilde{\lambda}$ be the pullback of $\lambda$ on $\widetilde{S}$. 
Proposition 6.20 For every $\tilde{p}, \tilde{q} \in \tilde{S} \backslash \tilde{\lambda}$ we have

$$
\theta_{n} d_{\widetilde{h}_{n}^{\star}}(\tilde{p}, \widetilde{q}) \rightarrow \iota(\widetilde{\alpha}, \tilde{\lambda}),
$$

where $\tilde{\alpha}$ is any smooth path in $\tilde{S}$ joining $\tilde{p}$ to $\tilde{q}$ and meeting each leaf of $\tilde{\lambda}$ at most once and transversely. Moreover, the convergence is uniform on compact subsets of $\tilde{S} \backslash \tilde{\lambda}$.

Theorem 1.14 is a direct consequence of this statement, so that its proof will be a consequence of the proof of this proposition.

First, notice that it is sufficient to prove Proposition 6.20 after rescaling $\theta_{n}$ and $\lambda$ by some arbitrary factor. In particular, we may assume the projective surface $\operatorname{Gr}_{\lambda / 2}(S, h)$ is quasi-Fuchsian. This technical assumption will simplify some steps of the proof.

First we show the following.

\section{Lemma 6.21}

$$
\liminf \theta_{n} d_{\widetilde{h}_{n}^{\star}}(\tilde{p}, \tilde{q}) \geq \iota(\tilde{\alpha}, \tilde{\lambda})
$$

Proof Let $M_{n}$ be the hyperbolic end introduced in Section 6.1 with $h_{n}=h$ and let $\sigma_{n}: \widetilde{S} \rightarrow \widetilde{M}_{n}$ be the lifting of the embedding $\bar{\sigma}_{n}: S \rightarrow M_{n}$. By Proposition 6.2, $M_{n}$ converges to the hyperbolic end facing $G r_{\lambda / 2}(S, h)$. In particular, we may assume that $M_{n}$ are all quasi-Fuchsian, so that $\widetilde{M}_{n}$ is a concave region of $\mathbb{H}^{3}$.

By Corollary 6.18, the family of embeddings $\left(\sigma_{n}\right)_{n \in \mathbb{N}}$ converges to the bending map $\sigma_{\infty}:(\tilde{S}, \tilde{h}) \rightarrow \mathbb{H}^{3}$, with bending lamination $\tilde{\lambda} / 2$.

Let $\widetilde{r}_{n}^{\star}: \widetilde{S}_{n}^{\star} \rightarrow \partial \widetilde{M}_{n}^{\star}$ be the 1-Lipschitz map defined in Lemma 6.5. We denote by $\Xi_{n}(\tilde{p})$ the plane in $\mathbb{H}^{3}$ corresponding to $\widetilde{r}_{n}^{\star}\left(\sigma_{n}^{\star}(\tilde{p})\right)$ and by $\Xi_{n}(\widetilde{q})$ the plane corresponding to $\widetilde{r}_{n}^{\star}\left(\sigma_{n}^{\star}(\widetilde{q})\right)$.

We have that $\Xi_{n}(\tilde{p})$ and $\Xi_{n}(\widetilde{q})$ are both support planes of $\partial \widetilde{M}_{n}$. Now let us put $\iota_{n}=d_{\partial \widetilde{M}_{n}^{\star}}\left(\widetilde{r}_{n}^{\star}\left(\sigma_{n}^{\star}(\tilde{p})\right), \widetilde{r}_{n}^{\star}\left(\sigma_{n}^{\star}(\widetilde{q})\right)\right)$.

Since $\widetilde{r}_{n}$ decreases the distances we deduce that

$$
\iota_{n} \leq d_{I I I_{n}}\left(\sigma_{n}(\widetilde{p}), \sigma_{n}(\widetilde{q})\right) \sim \frac{\theta_{n}}{2} d_{\widetilde{h}_{n}^{\star}}(\tilde{p}, \widetilde{q}) .
$$

We claim that $\Xi_{n}(\tilde{p})$ and $\Xi_{n}(\widetilde{q})$ converge to the support planes of $\partial \widetilde{M}_{\infty}$ at $\sigma_{\infty}(\widetilde{p})$ and $\sigma_{\infty}(\widetilde{q})$ respectively. 
Let us explain how the conclusion follows. The claim ensures that it is possible to construct a sequence of $\operatorname{arcs} \widetilde{\alpha}_{n}:[0,1] \rightarrow \partial \widetilde{M}_{n}$ such that

- each $\widetilde{\alpha}_{n}$ intersects each leaf of $\tilde{\lambda}_{n}$ at most once and transversely; moreover endpoints of $\widetilde{\alpha}_{n}$ are contained in $\partial \widetilde{M}_{n} \backslash \tilde{\lambda}_{n}$;

- endpoints of $\widetilde{\alpha}_{n}$ are close to $\Xi_{n}(\tilde{p})$ and $\Xi_{n}(\widetilde{q})$ in the sense that we have that $d_{\partial} \widetilde{M}_{n}^{\star}\left(\widetilde{\alpha}_{n}^{\star}(0), r_{n}^{\star}\left(\sigma_{n}^{\star}(\widetilde{p})\right)\right)$ and $d_{\partial \widetilde{M}_{n}^{\star}}\left(\widetilde{\alpha}_{n}^{\star}(1), r_{n}^{\star}\left(\sigma_{n}^{\star}(\widetilde{q})\right)\right)$ converge to 0 ;

- $\tilde{\alpha}_{n}$ converges to a path $\tilde{\alpha}_{\infty}$ connecting the stratum of $\partial \widetilde{M}_{\infty} \backslash \tilde{\lambda}$ containing $\sigma_{\infty}(\widetilde{p})$ with the stratum containing $\sigma_{\infty}(\widetilde{q})$.

Thus we have $\iota(\tilde{\lambda} / 2, \tilde{\alpha})=\iota\left(\tilde{\lambda} / 2, \tilde{\alpha}_{\infty}\right)=\lim _{n} \iota\left(\tilde{\lambda}_{n}, \tilde{\alpha}_{n}\right)$. By (32), we have that $\iota\left(\tilde{\lambda}_{n}, \widetilde{\alpha}_{n}\right)=d_{\partial \widetilde{M}_{n}}\left(\widetilde{\alpha}_{n}^{\star}(0), \tilde{\alpha}_{n}^{\star}(1)\right)$, so we conclude that

$$
\iota(\tilde{\lambda} / 2, \tilde{\alpha})=\lim _{n} \iota_{n}
$$

and the conclusion of the Lemma follows from Equation (51).

In order to prove the claim recall that $\widetilde{r}_{n}^{\star}$ sends any point $\widetilde{x}^{\star}$ of $\widetilde{S}_{n}^{\star}$ to a point in $\partial \widetilde{M}_{n}^{\star}$ that lies in the past of $\tilde{x}^{\star}$ (Lemma 6.5).

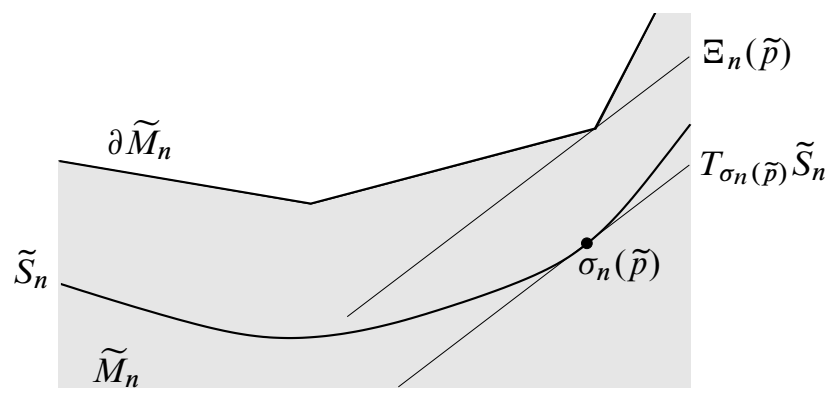

Figure 4: Convergence of the support planes

This implies $\Xi_{n}(\tilde{p})$ and $T_{\sigma_{n}(\tilde{p})} \widetilde{S}_{n}$ are disjoint planes. In particular we deduce that $\Xi_{n}(\tilde{p})$ separates $\sigma_{n}(\tilde{p})$ from $\partial \widetilde{M}_{n}$ (see Figure 4). This easily implies $\Xi_{n}(\tilde{p})$ converges to the support plane of $\partial \widetilde{M}_{\infty}$ at $\sigma_{\infty}(\widetilde{p})$ (that is unique by our assumption that $\tilde{p}$ does not lie on $\tilde{\lambda})$. Analogously $\Xi_{n}(\widetilde{q})$ converges to the support plane of $\partial \widetilde{M}_{\infty}$ at $\sigma_{\infty}(\widetilde{q})$.

To conclude the proof of Proposition 6.20 we need to show that

$$
\lim \sup \theta_{n} d_{\tilde{h}_{n}^{\star}}(\tilde{p}, \tilde{q}) \leq \iota(\tilde{\alpha}, \tilde{\lambda}) .
$$

In order to estimate $\theta_{n} d_{\widetilde{h}_{n}^{\star}}(\tilde{p}, \widetilde{q})$, we need the following result. 
Lemma 6.22 Let $\tilde{U}$ be any convex smooth surface in $\mathbb{H}^{3}$ and let $\tilde{x}, \tilde{y} \in \tilde{U}$ such that the support planes $\Xi_{\tilde{x}}$ and $\Xi_{\tilde{y}}$ at $\tilde{x}$ and $\tilde{y}$ intersect. Then the distance between $\tilde{x}$ and $\tilde{y}$ with respect to the third fundamental form of $\tilde{U}$ is less than the angle between $\Xi_{\tilde{x}}$ and $\Xi_{\tilde{y}}$.

We first prove a 2-dimensional version of this lemma.

Sublemma 6.23 Let $\tilde{\varsigma}$ be a convex curve in $\mathbb{H}^{2}$ joining two points $\tilde{x}, \tilde{y}$. Suppose that the support lines $\tilde{l}_{\tilde{x}}$ and $l_{\tilde{y}}$ at $\tilde{x}$ and $\tilde{y}$ intersect. Then the angle they form is bigger than the integral of the curvature of $\widetilde{\zeta}$.

Proof By Gauss-Bonnet formula, the area bounded by $l_{\tilde{x}}, l_{\tilde{y}}$ and $\tilde{\zeta}$ is equal to the difference between the wanted angle and the integral of the curvature.

Proof of Lemma 6.22 First we translate the condition that planes intersect in terms of a condition of dual points $\tilde{x}^{\star}$ and $\tilde{y}^{\star}$. Recalling that $\tilde{x}^{\star}$ and $\tilde{y}^{\star}$ are unit vector orthogonal to the planes (and pointing in the concave region bounded by $\tilde{U}$ ), we easily derive that $\Xi_{\tilde{x}}$ and $\Xi_{\tilde{y}}$ intersect if and only if the segment $\widetilde{\kappa}^{\star}$ joining $\tilde{x}^{\star}$ to $\tilde{y}^{\star}$ in $\mathrm{dS}^{3}$ is spacelike, in which case the angle between $\Xi_{\tilde{x}}$ and $\Xi_{\tilde{y}}$ coincides with the length of $\widetilde{\kappa}^{\star}$.

Let $\Pi$ be a timelike plane containing $\tilde{\kappa}^{\star}$. Since $\tilde{U}^{\star}$ is an achronal surface, $\tilde{U}^{\star} \cap \Pi$ is a curve containing $\tilde{x}^{\star}$ and $\tilde{y}^{\star}$. Let $\tilde{\zeta}^{\star}$ be the segment on $\tilde{U}^{\star} \cap \Pi$ connecting $\tilde{x}^{\star}$ to $\tilde{y}^{\star}$. Clearly, the length of $\widetilde{\zeta}^{\star}$ is greater than the distance on $\tilde{U}^{\star}$ between $\tilde{x}^{\star}$ and $\tilde{y}^{\star}$. In order to conclude, it is sufficient to prove that the length of $\widetilde{\zeta}^{\star}$ is less than the length of $\widetilde{\kappa}^{\star}$.

Notice that this is a 2 -dimensional problem. In fact, let $\Upsilon$ be the timelike linear 3-space in $\mathbb{R}^{3,1}$ such that $\Pi=\Upsilon \cap \mathrm{dS}^{3}$. The intersection $\Upsilon \cap \mathbb{H}^{3}$ is a hyperbolic plane denoted by $\Lambda$. Points on $\Pi$ correspond to planes of $\mathbb{H}^{3}$ that orthogonally meet $\Lambda$. In particular, points on $\Pi$ bijectively correspond to lines on $\Lambda$ and points of $\Lambda$ correspond to spacelike lines of $\Pi$.

Consider the curve $\widetilde{\varsigma}$ on $\Lambda$ of points corresponding to support lines of $\widetilde{\zeta}^{\star}$. Notice that support lines at the endpoints of $\widetilde{\zeta}$ are $\Xi_{\tilde{x}} \cap \Lambda$ and $\Xi_{\tilde{y}} \cap \Lambda$, so the angles these lines form is equal to the angle between $\Xi_{\tilde{x}}$ and $\Xi_{\tilde{y}}$ and it is equal to the length of $\widetilde{\kappa}^{\star}$. On the other hand the length of $\widetilde{\zeta}^{\star}$ is equal to the integral of the curvature of $\widetilde{\zeta}$. Thus the conclusion follows from Sublemma 6.23.

Given any geodesic $\widetilde{\varsigma} \in(\tilde{S}, \tilde{h})$, we say that $\sigma_{n}(\widetilde{\zeta})$ is a short path if the support planes at $\sigma_{n}(\widetilde{\zeta}(0))$ and $\sigma_{n}(\widetilde{\zeta}(1))$ intersect. If $\sigma_{n}(\widetilde{\zeta})$ is a short path, then we denote by $\eta_{n}(\widetilde{\zeta}) \in(0, \pi)$ the angle between the support planes at its endpoints. 
Analogously, we say that $\sigma_{\infty}(\widetilde{\zeta})$ is a short path if the endpoints of $\sigma_{\infty}(\widetilde{\zeta})$ are outside the bending lamination and the corresponding support planes intersect. In this case, $\eta_{\infty}(\widetilde{\zeta})$ is the angle between such planes.

Clearly, if $\sigma_{\infty}(\widetilde{\varsigma})$ is a short path, then $\sigma_{n}(\widetilde{\varsigma})$ is definitively a short path and we have that $\eta_{n}(\widetilde{\varsigma}) \rightarrow \eta_{\infty}(\widetilde{\varsigma})$.

Lemma 6.24 There exists $\epsilon_{0}$ such that if $\tilde{\alpha}:[0,1] \rightarrow \widetilde{S}$ is a geodesic path for $\tilde{h}$ of length less than $\epsilon_{0}$, then

- $\sigma_{\infty}(\widetilde{\alpha})$ is a short path,

- $\iota(\tilde{\alpha}, \tilde{\lambda}) \leq \eta_{\infty}(\widetilde{\alpha}) \leq\left(1+\ell_{\tilde{h}}(\widetilde{\alpha})\right) \iota(\tilde{\alpha}, \tilde{\lambda})$.

Proof The first point easily follows, since $\widetilde{S}_{\infty}$ is invariant under the action of a cocompact group of isometries of $\mathbb{H}^{3}$.

About the second point, notice that the first inequality is given by Lemma 6.22. The second inequality is more subtle. Choosing $\epsilon_{0}$ sufficiently small, we can suppose that either $\widetilde{\alpha}$ intersects only one isolated leaf of $\widetilde{\lambda}$ or it intersects no isolated leaf. In the first case the second inequality is obvious.

Up to taking a smaller $\epsilon_{0}$, we may suppose that, if $T$ is a hyperbolic triangle with an edge $e$ of length $l \leq \epsilon_{0}$ and the angles $\vartheta_{1}, \vartheta_{2}$ adjacent to $e$ less than $\pi / 4$, then the area of $T$ is less than $l \vartheta_{2}$. Now take a geodesic $\tilde{\alpha}$ on $\tilde{S}$ of length less than $\epsilon$ which does not intersect the isolated leaves of the lamination. Taking any subdivision $\widetilde{\alpha}(0)=\widetilde{p}_{1}, \ldots, \widetilde{p}_{m+1}=\widetilde{\alpha}(1)$ of $\tilde{\alpha}$, we consider the support planes $\Xi_{i}$ of $\sigma_{\infty}(\tilde{S})$ at $\sigma_{\infty}\left(\tilde{p}_{i}\right)$. If the subdivision is sufficiently fine, then the angles $\vartheta_{i}$ between $\Xi_{i}$ and $\Xi_{i+1}$ are less than $\pi / 4$. Now consider the boundary $\partial K$ of the convex set $K$ obtained by intersecting the half-spaces bounded by $\Xi_{1}, \ldots, \Xi_{m+1}$ and containing $\sigma_{\infty}(\tilde{S})$. Notice that $\partial K$ is a finite bent surface: indeed, its bending lines are $\Xi_{i} \cap \Xi_{i+1}$ for every $i$ such that $\Xi_{i}$ and $\Xi_{i+1}$ are different. We claim that $\eta_{\infty}(\widetilde{\alpha}) \leq\left(1+\ell_{\tilde{h}}(\underset{\tilde{\alpha}}{\tilde{\alpha}})\right) \sum \vartheta_{i}$. Taking a sequence of arbitrary fine subdivisions, we have that $\sum \vartheta_{i} \rightarrow \iota(\tilde{\alpha}, \tilde{\lambda})$, so the second inequality follows from the claim.

In order to prove the claim we use an inductive argument. Notice that, if $\Xi_{1}, \Xi_{2}, \Xi_{3}$ are distinct, then $\Xi_{1} \cap \Xi_{2} \cap \Xi_{3}=\varnothing$. Thus, there is a plane $\Lambda$ orthogonal to all of them. The triangle $T$ obtained by intersecting $\Lambda \cap \Xi_{1}, \Lambda \cap \Xi_{2}$ and $\Lambda \cap \Xi_{3}$ has angles $\vartheta_{1}, \vartheta_{2}$ and $\pi-\bar{\vartheta}_{1}$, where $\bar{\vartheta}_{1}$ is the angle formed by $\Xi_{1}$ and $\Xi_{3}$.

Notice that the length of the edge between $\vartheta_{1}$ and $\vartheta_{2}$ is less than the distance between $\tilde{p}_{1}$ and $\tilde{p}_{2}$, and so it is smaller than $\epsilon_{0}$. We conclude that the area of $T$ is less than $d_{\tilde{h}}\left(\tilde{p}_{1}, \tilde{p}_{2}\right) \vartheta_{2}$. In particular, we deduce that $\bar{\vartheta}_{1} \leq \vartheta_{1}+\vartheta_{2}\left(1+d_{\tilde{h}}\left(\tilde{p}_{1}, \tilde{p}_{2}\right)\right)$. 

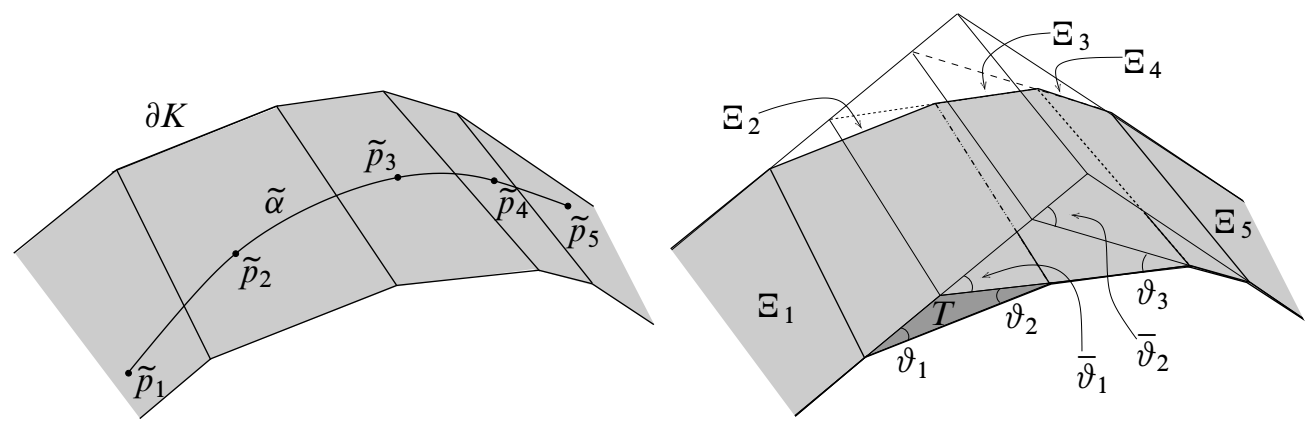

If at least two planes among $\Xi_{1}, \Xi_{2}, \Xi_{3}$ coincide, then the area of $T$ is zero, and so $\bar{\vartheta}_{1}=\vartheta_{1}+\vartheta_{2} \leq \vartheta_{1}+\vartheta_{2}\left(1+d_{\tilde{h}}\left(\tilde{p}_{1}, \tilde{p}_{2}\right)\right)$.

Apply now the same argument to the planes $\Xi_{1}, \Xi_{3}, \Xi_{4}$. If $\bar{\vartheta}_{2}$ is the angle formed by $\Xi_{1}$ and $\Xi_{4}$, then $\bar{\vartheta}_{2} \leq \bar{\vartheta}_{1}+\vartheta_{3}\left(1+d_{\tilde{h}}\left(\tilde{p}_{1}, \tilde{p}_{3}\right)\right) \leq \bar{\vartheta}_{1}+\vartheta_{2}\left(1+d_{\tilde{h}}\left(\tilde{p}_{1}, \tilde{p}_{2}\right)\right)+$ $\vartheta_{3}\left(1+d_{\tilde{h}}\left(\tilde{p}_{1}, \tilde{p}_{3}\right)\right)$. Iterating this procedure, we deduce the angle between $\Xi_{1}$ and $\Xi_{m}$ is bounded by $\vartheta_{1}+\vartheta_{2}\left(1+d_{\tilde{h}}\left(\tilde{p}_{1}, \tilde{p}_{2}\right)+\vartheta_{3}\left(1+d_{\tilde{h}}\left(\tilde{p}_{1}, \tilde{p}_{3}\right)\right)+\cdots+\vartheta_{m}\left(1+d_{\tilde{h}}\left(\tilde{p}_{1}, \tilde{p}_{m}\right)\right)\right.$ and this quantity is less than $\left(1+\ell_{\widetilde{h}}(\widetilde{\alpha})\right) \sum \vartheta_{i}$.

We can now prove (52). Fix $\epsilon<\epsilon_{0}$ and subdivide the geodesic $\tilde{\alpha}$ joining $\tilde{p}$ to $\tilde{q}$ into segments $\tilde{\alpha}_{i}$ of length less than $\epsilon$. Let $\tilde{p}=\widetilde{p}_{1}, \widetilde{p}_{2}, \ldots, \widetilde{p}_{m+1}=\widetilde{q}$ be endpoints of such subdivision.

For $n$ large, $\sigma_{n}\left(\widetilde{\alpha}_{i}\right)$ are short paths and so by Lemma 6.22

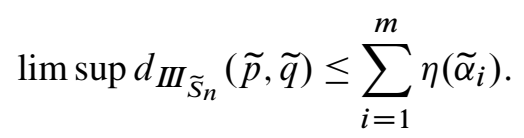

On the other hand, applying Lemma 6.24 we deduce that

$$
\sum_{i=1}^{m} \eta\left(\tilde{\alpha}_{i}\right) \leq \iota(\tilde{\alpha}, \tilde{\lambda})(1+\epsilon) .
$$

The uniform convergence follows from the fact that the whole argument works as well, if we consider sequences of points $\widetilde{p}_{n} \rightarrow \tilde{p}$ and $\widetilde{q}_{n} \rightarrow \widetilde{q}$ belonging to a compact subset of $\widetilde{S} \backslash \tilde{\lambda}$. This concludes the proof of Proposition 6.20.

\section{Behavior of the centers}

In this section we want to discuss the behavior of the centers $c_{n}$ when $h$ is fixed and $h_{n}^{\star} \rightarrow[\lambda]$ in Thurston compactification. Our aim is to prove that the limit point(s) of $c_{n}$ 
does not only depend on $h$ and $[\lambda]$ but also on the sequence $h_{n}^{\star}$. As a consequence, we will see that the analog of Theorem 1.12 does not hold if the sequence of centers $\left(c_{n}\right)$ converges to a projective measured lamination; see Corollary 7.3.

Fix a hyperbolic metric $h$ on $S$ and let $c$ be a point in the boundary of the augmented Teichmüller space of $S$. Then let $c^{\prime}$ be the unique complete hyperbolic metric of finite area on $S \backslash \Gamma$ in the conformal class $c$, where $\Gamma$ is the disjoint union of simple closed curves $\gamma_{1}, \ldots, \gamma_{l}$. Up to isotopy, we can assume that $\gamma_{i}$ is a geodesic for $h$ and we denote by $\ell_{i}$ the $h$-length of $\gamma_{i}$. We will also denote by $\bar{S}$ the surface obtained from $S$ by collapsing each $\gamma_{i}$ to a node $v_{i}$.

We recall a construction of an infinite energy harmonic map $f:\left(S \backslash \Gamma, c^{\prime}\right) \rightarrow(S \backslash \Gamma, h)$ by Wolf [44]. For every $i=1, \ldots, l$, choose a sequence $s_{i, n} \rightarrow+\infty$ with $s_{i, n}>1$. Let $U_{i,+}(s)$ and $U_{i,-}(s)$ be the cusps of $\left(S \backslash \Gamma, c^{\prime}\right)$ bounded by horocycles of length $1 / s$ near $v_{i}$ and let $U_{i}(s):=U_{i,+}(s) \cup U_{i,-}(s)$ and $\bar{U}_{i}(s):=U_{i}(s) \cup \gamma_{i}$.

Fix an isometry $\xi_{i, \bullet}: U_{i, \bullet}(1) \rightarrow U /(0, y) \sim(1, y)$ with $U=[0,1] \times[1,+\infty) \subset \mathbb{H}^{2}$ and put on $U_{i, \bullet}(1)$ the flat metric $\left|\Psi_{i}\right|$ induced by the quadratic differential $\Psi_{i}=\xi_{i, \bullet}^{*}\left(d z^{2}\right)$.

Define a new metric $\check{c}$ on $S \backslash \Gamma$ which

- agrees with $c^{\prime}$ outside $\bigcup_{i} U_{i}(1)$ and with $\left|\Psi_{i}\right|$ on each $U_{i, \bullet}(1)$,

- is in the conformal class $c$,

- $\check{c}$ is smooth away from $\partial U_{i}(1)$.

Let $U_{i, \bullet}^{n}(s)$ be the annulus $U_{i, \bullet}(s) \backslash U_{i, \bullet}\left(s_{i, n}\right)$ and call $U_{i}^{n}(s):=U_{i,+}^{n}(s) \cup U_{i,-}^{n}(s)$. We denote by $\xi_{i, \bullet}^{n}: U_{i, \bullet}^{n}(1) \rightarrow U / \sim$ the restriction of $\xi_{i, \bullet}$.

Then $S_{n}$ is obtained from $S$ by removing $\bar{U}_{i}\left(s_{i, n}\right)$ from the cusps adjacent to $\gamma_{i}$. Gluing the seams together, we obtain a compact surface $\bar{S}_{n}$ with quadratic differentials $\Psi_{i}^{n}$ on $\bar{U}_{i}^{n}(1)$ obtained restricting $\Psi_{i}$, with distinguished geodesics $\gamma_{i}^{n}$ corresponding to the seams and collars $\bar{U}_{i}^{n}(s)=U_{i}^{n}(s) \cup \gamma_{i}^{n}$. We will also define $\xi_{i}^{n}: \bar{U}_{i}^{n}(1) \rightarrow U / \sim$ as

$$
\xi_{i}^{n}(p)= \begin{cases}\xi_{i,+}^{n}(p) & \text { if } p \in \bar{U}_{i,+}^{n}, \\ 1+2 i\left(s_{i, n}+1\right)-\xi_{i,-}^{n}(p) & \text { if } p \in \bar{U}_{i,-}^{n} .\end{cases}
$$

Notice that the metric $\check{c}_{n}$ induced by $\check{c}$ on $\bar{S}_{n}$ determines a point $c_{n}$ in $\mathcal{T}(S)$ : we will denote by $c_{n}^{\prime}$ the hyperbolic metric in the conformal class $c_{n}$.

Notice that, hidden in this construction, there is an arbitrary choice of twists associated to the gluings or, equivalently, to the charts $\xi_{i, \bullet}$.

Call $f_{n}:\left(\bar{S}_{n}, c_{n}\right) \rightarrow(S, h)$ the unique harmonic map in the given homotopy class [12]. 
Theorem 7.1 (Wolf [44]) Up to subsequences, $f_{n}$ converges $C^{2, \alpha}$ to a harmonic map $f:(S \backslash \Gamma, c) \rightarrow(S \backslash \Gamma, h)$ on the compact subsets of $S \backslash \Gamma$.

The Hopf differential $\Phi$ of $f$ looks like $\Phi=\left(\ell_{i}^{2} / 4+O\left(e^{-2 \pi y \circ \xi_{i} \bullet}\right)\right) \Psi_{i}$ in each cusp $U_{i, \bullet}(1)$ of $(S \backslash \Gamma, c)$. Moreover, the energy density $e(f ; \check{c}, h) \rightarrow \ell_{i}^{2} / 2$ and the holomorphic energy density $\mathcal{H}(f ; \check{c}, h) \rightarrow \ell_{i}^{2} / 4$ as $y \circ \xi_{i, \bullet} \rightarrow+\infty$.

We will assume throughout this section that we have already extracted a good subsequence (which we will still call $f_{n}$ ) so that the above theorem holds.

Now let $h_{n}^{\star}$ be the metric on $S$ antipodal to $h$ with respect to $\left(f_{n}\right)_{*} c_{n}$. Because of the theorem, $h_{n}^{\star}$ converges to some $h^{\star}$ smoothly away from $\Gamma$.

Proposition 7.2 Let $1=b_{1}=\cdots=b_{r}>b_{r+1} \geq \cdots \geq b_{l}>0$ and put $s_{i, n}=\left(a_{i} / \ell_{i}\right) t_{n}^{b_{i}}$, where $a_{i}>0$ and $t_{n} \rightarrow+\infty$. Then, up to subsequences, $c_{n} \rightarrow\left[b_{1} \gamma_{1}+\cdots+b_{l} \gamma_{l}\right]$ and $h_{n}^{\star} \rightarrow\left[a_{1} \gamma_{1}+\cdots+a_{r} \gamma_{r}\right]$ in Thurston compactification of $\mathcal{T}(S)$.

Corollary 7.3 If $h$ is fixed and $h_{n}^{\star} \rightarrow[\lambda]$ in Thurston compactification, then $c_{n}$ does not necessarily converge to $[\lambda]$. If $h$ is fixed and $c_{n} \rightarrow[\lambda]$ in Thurston compactification, then $h_{n}^{\star}$ does not necessarily converge to $[\lambda]$ and so the cyclic flow centered at $c_{n}$ does not necessarily converge to an earthquake along $\lambda$ (with any normalization).

In order to prove Proposition 7.2, we need to estimate the transversal length $\operatorname{trl}_{\gamma_{i}}\left(c_{n}^{\prime}\right)$, that is, the width of a standard $c_{n}^{\prime}$-collar of $\gamma_{i}^{n}$ bounded by hypercycles of length 1 .

Lemma 7.4 The extremal length of $\gamma_{i}$ at $c_{n}$ satisfies

$$
\frac{1}{C_{1}+2\left(a_{i} / \ell_{i}\right) t_{n}^{b_{i}}} \leq \operatorname{Ext}_{\gamma_{i}}\left(c_{n}\right) \leq \frac{\ell_{i}}{2 a_{i} t_{n}^{b_{i}}}
$$

and so $\operatorname{trl}_{\gamma_{i}}\left(c_{n}^{\prime}\right) \asymp 2 b_{i} \log t_{n}$.

Proof By construction, $\left(\bar{U}_{i}^{n}(1), \check{c}_{n}\right)$ contains a flat cylinder of circumference 1 and height $2\left(a_{i} / \ell_{i}\right) t_{n}^{b_{i}}$ and so the extremal length satisfies

$$
\operatorname{Ext}_{\gamma_{i}}\left(c_{n}\right) \leq \frac{\ell_{i}}{2 a_{i} t_{n}^{b_{i}}} .
$$

On the other hand, consider a metric $e^{2 u} \check{c}_{n}$ on $\bar{S}_{n}$ which is in the conformal class $c_{n}$, which agrees with $\check{c}_{n}$ on $\bar{S}_{n} \backslash \bigcup_{j \neq i} \bar{U}_{j}^{n}$ and such that the $e^{2 u} \check{c}_{n}$-area of $\bar{U}_{j}^{n}$ is bounded by a fixed constant for $j \neq i$ and the distance between the two boundary components of $\bar{U}_{j}^{n}$ is at least 1 . For instance, one can define $e^{u}$ be constantly $1 / s_{j, n}$ on the 
regions $\bar{U}_{j}^{n}(2)$ for all $j \neq i$, which interpolates between 1 and $1 / s_{j, n}$ on $\bar{U}_{j}^{n}(1) \backslash \bar{U}_{j}^{n}(2)$ for $j \neq i$, and which is constantly 1 elsewhere.

Then $\ell_{\gamma_{i}}\left(e^{2 u} \check{c}_{n}\right)=1$ and $\operatorname{Area}_{e} e^{2 u} \check{c}_{n}(S) \leq C_{1}+2\left(a_{i} / \ell_{i}\right) t_{n}^{b_{i}}$ where $C_{1}$ is a constant that depends only on $\chi(S)$ and $k$. Hence,

$$
\operatorname{Ext}_{\gamma_{i}}\left(c_{n}\right) \geq \frac{1}{C_{1}+2\left(a_{i} / \ell_{i}\right) t_{n}^{b_{i}}} .
$$

As $\operatorname{Ext}_{\gamma_{i}}\left(c_{n}\right) \rightarrow 0$, Maskit's estimate (see Maskit [27]) gives $\ell_{\gamma_{i}}\left(c_{n}^{\prime}\right) \asymp \pi \operatorname{Ext}_{\gamma_{i}}\left(c_{n}\right)$ and so $\operatorname{trl}_{\gamma_{i}}\left(c_{n}^{\prime}\right) \asymp-2 \log \ell_{\gamma_{i}}\left(c_{n}^{\prime}\right) \asymp 2 b_{i} \log t_{n}$.

For each $i$, fix an open neighbourhood $A_{i} \subset(\bar{S}, c)$ of $v_{i}$ whose closure does not contain any zero of $\Phi$, and such that $\left(A_{i} \backslash v_{i},|\Phi|\right)$ is the union of two annuli. Moreover, choose standard $h$-collars $R_{i} \subset(S, h)$ around $\gamma_{i}$ such that $R_{i} \subset f\left(A_{i}\right)$. By Wolf's construction (see [44]), outside $\bigcup_{i} f^{-1}\left(R_{i}\right)$ the Hopf differential $\Phi_{n}$ of $f_{n}$ converges $C^{1, \alpha}$ to $\Phi$.

Here we recall that, by definition,

$$
h=2\left(f_{n}\right)_{*} \operatorname{Re}\left(\Phi_{n}\right)+e\left(f_{n} ; \check{c}_{n}, h\right) \check{c}_{n},
$$

where $e\left(f_{n} ; \check{c}_{n}, h\right)$ is the energy density; moreover, by Equation (2) in Section 3.4,

$$
\left(f_{n}\right)_{*} \operatorname{Re}\left(\Phi_{n}\right)=\frac{1}{4} h\left(\left(E-b_{n}^{2}\right) \bullet, \bullet\right),
$$

and so $b_{n}$ converges $C^{1, \alpha}$ to $b$ outside $\bigcup_{i} R_{i}$.

Notice that the horizontal (resp. vertical) directions of $\Phi_{n}$ are exactly the eigenspaces of $b_{n}$ corresponding to the smaller (resp. bigger) eigenvalue.

Lemma 7.5 Fix a small $\varepsilon>0$. Up to shrinking $R_{i}$ and for $n$ large enough,

$$
\begin{gathered}
\left|\frac{4}{\ell_{i}^{2}} \frac{\Phi_{n}}{\Psi_{i}^{n}}-1\right|<\varepsilon^{2}, \\
\left|\frac{2}{\ell_{i}^{2}} e\left(f_{n} ; \check{c}_{n}, h\right)-1\right|<2 \varepsilon^{2},
\end{gathered}
$$

in every $f_{n}^{-1}\left(R_{i}\right)$.

Proof Up to shrinking $R_{i}$, we can assume that $R_{i} \subset f_{n}\left(\bar{U}_{i}^{n}(3)\right)$ and there we have

$$
\left|\frac{4}{\ell_{i}^{2}} \frac{\Phi}{\Psi_{i}}-1\right|<\varepsilon^{2} / 2, \quad\left|\frac{4}{\ell_{i}^{2}} \mathcal{H}(f ; \check{c}, h)-1\right|<\varepsilon^{2} / 2,
$$

where $\mathcal{H}(f ; \check{c}, h)=\frac{1}{2}\|\partial f\|^{2}$ is the holomorphic energy density of $f$. 
As $\left.\left.f_{n}^{-1}\right|_{\partial R_{i}} \rightarrow f^{-1}\right|_{\partial R_{i}}$, for $n$ large enough $f_{n}^{-1}\left(\partial R_{i}\right)$ is contained inside $\bar{U}_{i}^{n}(2)$. Moreover, we have that $\left(\xi_{i, \bullet}^{n}\right)_{*} \Phi_{n} \rightarrow\left(\xi_{i, \bullet}\right)_{*} \Phi$ in a compact neighbourhood $K_{i, \bullet}$ of $\xi_{i, \bullet}^{n}\left(f_{n}^{-1}\left(\partial R_{i}\right) \cap U_{i, \bullet}^{n}\right)$ and so

$$
\frac{4}{\ell_{i}^{2}}\left|\left(\xi_{i, \bullet}^{n}\right)_{*} \frac{\Phi_{n}}{\Psi_{i}^{n}}-\left(\xi_{i, \bullet}\right)_{*} \frac{\Phi}{\Psi_{i}}\right|<\varepsilon^{2} / 2
$$

in $K_{i, \bullet}$. Thus,

$$
\frac{4}{\ell_{i}^{2}}\left|\left(\xi_{i}^{n}\right) * \frac{\Phi_{n}}{\Psi_{i}^{n}}-1\right|<\varepsilon^{2}
$$

in $K_{i, \bullet}$. Because $\xi_{i, \bullet}^{n}$ is holomorphic and $\Phi_{n} / \Psi_{i}^{n}$ is a holomorphic function on $f_{n}^{-1}\left(R_{i}\right)$, the same estimate holds in $f_{n}^{-1}\left(R_{i}\right)$ for $n$ large enough.

In a similar way, if $\mathcal{H}_{n}=\mathcal{H}\left(f_{n} ; \check{c}_{n}, h\right)$, then the energy density and the Jacobian of $f_{n}$ are given by

$$
e\left(f_{n} ; \check{c}_{n}, h\right)=\mathcal{H}_{n}+\frac{\left|\Phi_{n}\right|^{2}}{\left|\Psi_{i}^{n}\right|^{2} \mathcal{H}_{n}}, \quad \mathcal{J}\left(f_{n} ; \check{c}_{n}, h\right)=\mathcal{H}_{n}-\frac{\left|\Phi_{n}\right|^{2}}{\left|\Psi_{i}^{n}\right|^{2} \mathcal{H}_{n}}>0,
$$

which implies $\mathcal{H}_{n}>\left|\Phi_{n}\right| /\left|\Psi_{i}^{n}\right|>\left(\ell_{i}^{2} / 4\right)\left(1-\varepsilon^{2}\right)$ on $f_{n}^{-1}\left(R_{i}\right)$.

On the other hand (see [43], for instance),

$$
\Delta_{c_{n}} \log \mathcal{H}_{n}=2 \mathcal{J}_{n}>0,
$$

and so $\Delta_{c_{n}} \log \left(4 \mathcal{H}_{n} / \ell_{i}^{2}\right) \geq 0$ on $\bar{U}_{i}^{n}(2)$. As $\log \left(4 \mathcal{H}_{n} / \ell_{i}^{2}\right)<\varepsilon^{2} / 2$ on $\partial f_{n}^{-1}\left(R_{i}\right)$ for $n$ large, we obtain $\mathcal{H}_{n} \leq\left(\ell_{i}^{2} / 4\right)\left(1+\varepsilon^{2} / 2\right)$ on $f_{n}^{-1}\left(R_{i}\right)$ and so the wished estimate for $e\left(f_{n} ; \check{c}_{n}, h\right)$.

Thanks to the previous lemma, we can draw a few consequences.

Corollary 7.6 (i) The metric $h^{\star}$ has nodes at $\Gamma$ and $h_{n}^{\star} \rightarrow h^{\star}$ in the augmented Teichmüller space.

(ii) In the whole $f_{n}^{-1}\left(R_{i}\right)$ found in the above lemma, the bigger eigenvalue $\kappa$ of $b_{n}$ is greater than $1 / 2 \varepsilon$ for large $n$.

Proof As for (i), notice that $h_{n}^{\star}=-2 \operatorname{Re}\left(\Phi_{n}\right)+e\left(f_{n} ; \check{c}_{n}, h\right) \check{c}_{n}$. Because of Lemma 7.5, for every $\varepsilon>0$ there exists $R_{i}$ such that, for $n>n(\varepsilon)$, the $h_{n}^{\star}$-norm of $\left(f_{n} \circ\left(\xi_{i}^{n}\right)^{-1}\right)_{*} \partial_{x}$ is at most $2 \varepsilon \ell_{i}$ the $h_{n}$-norm of $\left(f_{n} \circ\left(\xi_{i}^{n}\right)^{-1}\right)_{*} \partial_{y}$ is at most $2 \varepsilon \ell_{i}$. Thus, $\ell_{\gamma_{i}}\left(h_{n}^{\star}\right) \leq 2 \varepsilon \ell_{i}$ and so $\ell_{\gamma_{i}}\left(h_{n}^{\star}\right) \rightarrow 0$. As $h_{n}^{\star} \rightarrow h^{\star}$ on the compact subsets of $S \backslash \Gamma$, we conclude $h^{\star}$ has nodes at $\gamma_{i}$ and the sequence converges in the augmented Teichmüller space. 
As for (ii), the identities seen in Section 3, Equation (2), $f_{n}^{*} h=2 \operatorname{Re}\left(\Phi_{n}\right)+e\left(f_{n} ; \check{c}_{n}, h\right) \check{c}_{n}$ and $f_{n}^{*} h\left(\left(E-b_{n}^{2}\right) \bullet, \bullet\right)=4 \operatorname{Re}\left(\Phi_{n}\right)$, give

$$
\frac{h\left(\left(E-b_{n}^{2}\right)\left(f_{n}\right)_{*} v,\left(f_{n}\right)_{*} v\right)}{h\left(\left(f_{n}\right)_{*} v,\left(f_{n}\right)_{*} v\right)}=\frac{4 \operatorname{Re}\left(\Phi_{n}\right)(v, v)}{2 \operatorname{Re}\left(\Phi_{n}\right)(v, v)+e\left(f_{n} ; \check{c}_{n}, h\right) \check{c}_{n}(v, v)}
$$

for any vector tangent $v$ to $\bar{U}_{i}^{n}(1)$. Choosing $v=\left(\xi_{i}^{n}\right)^{*} \partial_{y}$, we obtain

$$
\frac{h\left(\left(E-b_{n}^{2}\right)\left(f_{n}\right)_{*} v,\left(f_{n}\right)_{*} v\right)}{h\left(\left(f_{n}\right)_{*} v,\left(f_{n}\right)_{*} v\right)} \leq \frac{\ell_{i}^{2}\left(-1+3 \varepsilon^{2}\right)}{4 \ell_{i}^{2} \varepsilon^{2}}
$$

on $\bar{U}_{i}^{n}(1) \cap f_{n}^{-1}\left(R_{i}\right)$ and so $1-\kappa^{2} \leq\left(-1 / 4 \varepsilon^{2}\right)+1$ there, that is $\kappa \geq 1 / 2 \varepsilon$.

Up to subsequences, we can assume that $h_{n}^{\star}$ converges to a point $[\lambda]$ in Thurston boundary. Notice that $\lambda$ must be supported on $\gamma_{1} \cup \cdots \cup \gamma_{l}$ and so $\lambda=w_{1} \gamma_{1}+\cdots+w_{l} \gamma_{l}$ with $w_{1}, \ldots, w_{l} \geq 0$.

We will show that $[\lambda]=\left[a_{1} \gamma_{1}+\cdots+a_{r} \gamma_{r}\right]$, and so the result will not depend on the chosen subsequence.

Let $\left(\theta_{n}\right)_{n \in \mathbb{N}}$ be a sequence of positive numbers such that $\theta_{n} \ell_{h_{n}^{\star}} \rightarrow \iota(\lambda, \bullet)$.

Lemma 7.7 For every $i$, we have $\theta_{n} \ell_{i} s_{i, n} \rightarrow w_{i}$ as $n \rightarrow \infty$.

Proof Let $\varepsilon>0$. We can choose a collar $\gamma_{i} \subset R_{i} \subset S$ such that $\operatorname{tr}\left(b_{n}\right)>2 / \varepsilon$ on $R_{i}$ and $\left|\frac{4}{\ell_{i}^{2}} \Phi_{n}-\Psi_{i}^{n}\right|<\varepsilon^{2}\left|\Psi_{i}^{n}\right|$ on $f_{n}^{-1}\left(R_{i}\right)$ for $n$ large.

As $f_{n}^{-1} \rightarrow f^{-1}$ on $S \backslash \Gamma$, we can assume that there exists $\bar{y}>1$ such that for $n$ large, $\bar{U}_{n}^{i}(1) \supset f_{n}^{-1}\left(R_{i}\right) \supset \bar{U}_{n}^{i}(\bar{y})$.

By abuse of notation, denote just by $(x, y)$ the Euclidean coordinates on $R_{i}$ given by the parametrization $\xi_{i}^{n} \circ f_{n}$.

By writing the relation $4 R e\left(\Phi_{n}\right)=h\left(\left(E-b_{n}^{2}\right) \bullet, \bullet\right)$ in coordinates on $R_{i}$ and taking the determinant, we obtain

$$
(1+\eta(\varepsilon)) \ell_{i}^{2} d x \wedge d y=\sqrt{\operatorname{tr}\left(b_{n}\right)^{2}-4} \omega_{h},
$$

where $|\eta(\varepsilon)|<\varepsilon^{2}$ for $n$ large, by Lemma 7.5.

By Corollary 7.6(ii), $\left(1-\varepsilon^{2} / 2\right) \operatorname{tr}\left(b_{n}\right) \leq \sqrt{\operatorname{tr}\left(b_{n}\right)^{2}-4} \leq \operatorname{tr}\left(b_{n}\right)$ for $n$ large.

Multiplying by $\theta_{n}$ both hand sides of Equation (53) and integrating over $R_{i}$, we get

$$
\theta_{n}\left(1-\varepsilon^{2} / 2\right) \int_{\boldsymbol{R}_{i}} \operatorname{tr}\left(b_{n}\right) \omega_{h} \leq \theta_{n}(1+\eta(\varepsilon)) \ell_{i}^{2} \int_{\boldsymbol{R}_{i}} d x \wedge d y \leq \theta_{n} \int_{\boldsymbol{R}_{i}} \operatorname{tr}\left(b_{n}\right) \omega_{h} .
$$


Now,

$$
\theta_{n} \ell_{i}^{2}\left(s_{i, n}-\bar{y}\right) \leq \theta_{n} \ell_{i}^{2} \int_{R_{i}} d x \wedge d y \leq \theta_{n} \ell_{i}^{2} s_{i, n},
$$

and $\theta_{n} \int_{R_{i}} \operatorname{tr}\left(b_{n}\right) \omega_{h} \rightarrow w_{i} \ell_{i}$ by Proposition 6.16.

The result follows by comparing Equations (54) and (55).

Proof of Proposition 7.2 By Lemma 7.4 and Maskit's estimate, we immediately obtain that $c_{n} \rightarrow\left[b_{1} \gamma_{1}+\cdots+b_{l} \gamma_{l}\right]$.

By Lemma 7.7,

$$
\frac{w_{i}}{w_{j}}=\lim _{n} \frac{\ell_{i} s_{i, n}}{\ell_{j} s_{j, n}}=\lim _{n} \frac{a_{i} t_{n}^{b_{i}}}{a_{j} t_{n}^{b_{j}}},
$$

which shows that $[\lambda]=\left[a_{1} \gamma_{1}+\cdots+a_{r} \gamma_{r}\right]$.

\section{The landslide flow on the universal Teichmüller space}

In this section we show how the construction of the landslide flow $L$ extends to the universal Teichmüller space. We believe that this $S^{1}$ action on the product of two copies of the universal Teichmüller space can be of independent interest, but limit our investigations here to its definition and to checking that it is nontrivial.

\subsection{Minimal Lagrangian maps and the universal Teichmüller space}

The universal Teichmüller space $\mathcal{T}_{U}$ can be defined as the quotient of the group $\mathcal{Q S}$ of quasisymmetric homeomorphisms of the circle by composition on the left by projective transformations; see eg [14]. We will show here that the map $L$ defined above extends to a circle action $\mathcal{L}$ on $\mathcal{T}_{U} \times \mathcal{T}_{U}$. This is based on the following statement.

Theorem 8.1 [9] Let $\bar{\psi} \in \mathcal{Q S}$. There exists a unique quasiconformal minimal Lagrangian diffeomorphism $m: \mathbb{H}^{2} \rightarrow \mathbb{H}^{2}$ such that $\partial m=\underline{\psi}$.

We call $g$ the hyperbolic metric on $\mathbb{H}^{2}$, and $\nabla$ its Levi-Civita connection. It follows from the basic facts on minimal Lagrangian diffeomorphisms, as recalled in Section 1.5, that there exists a unique bundle morphism $b: T \mathbb{H}^{2} \rightarrow T \mathbb{H}^{2}$ such that

- $m^{*} g=g(b \bullet, b \bullet)$,

- $\operatorname{det}(b)=1$,

- $b$ is self-adjoint for $g$,

- $b$ satisfies the Codazzi equation: $d^{\nabla} b=0$.

Since $m$ is quasiconformal, $b$ has eigenvalues in $[\epsilon, 1 / \epsilon]$ for some $\epsilon>0$; see [9]. 


\subsection{An extension of $L$ to the universal Teichmüller space}

The construction of the $S^{1}$-action is based on the following definition and lemma.

Definition 8.2 Let $\bar{\psi} \in \mathcal{Q S}$ and let $e^{i \theta} \in S^{1}$. Let $m: \mathbb{H}^{2} \rightarrow \mathbb{H}^{2}$ be the unique quasiconformal minimal Lagrangian diffeomorphism such that $\partial m=\bar{\psi}$, and let $b: T \mathbb{H}^{2} \rightarrow T \mathbb{H}^{2}$ be as above. We call

$$
\beta_{\theta}=\cos (\theta / 2) E+\sin (\theta / 2) J b,
$$

and set $g_{\theta}:=g\left(\beta_{\theta} \bullet, \beta_{\theta} \bullet\right)$ and $g_{\theta}^{\star}=g\left(\beta_{\theta+\pi} \bullet, \beta_{\theta+\pi} \bullet\right)$.

Lemma 8.3 With the notation above, $g_{\theta}$ and $g_{\theta}^{\star}$ are complete hyperbolic metrics on $\mathbb{H}^{2}$. The identity map from $\left(\mathbb{H}^{2}, g_{\theta}\right)$ to $\left(\mathbb{H}^{2}, g_{\theta}^{\star}\right)$ is minimal Lagrangian and quasiconformal.

Sketch of the proof The fact that $g_{\theta}$ and $g_{\theta}^{\star}$ have curvature -1 follows from the same argument as in the proof of Proposition 1.7; we do not repeat it here. Moreover, the argument given in the proof of Theorem 1.10 also shows that the identity map from $\left(\mathbb{H}^{2}, g_{\theta}\right)$ to $\left(\mathbb{H}^{2}, g_{\theta}^{\star}\right)$ is minimal Lagrangian.

To check that the identity between $\left(\mathbb{H}^{2}, g\right)$ and $\left(\mathbb{H}^{2}, g_{\theta}\right)$ is quasiconformal, it is sufficient to prove that the eigenvalues of the bundle morphism ${ }^{t} \beta_{\theta} \cdot \beta_{\theta}$ are between $\epsilon^{\prime}$ and $1 / \epsilon^{\prime}$, for some $\epsilon^{\prime}>0$ depending on $\epsilon$. However $\operatorname{det}\left(\beta_{\theta}\right)=1$ by definition, so that ${ }^{t} \beta_{\theta} \cdot \beta_{\theta}$ has determinant 1 . To compute its trace, notice that

$$
{ }^{t} \beta_{\theta} \cdot \beta_{\theta}=\cos ^{2}(\theta / 2) E+\sin ^{2}(\theta / 2) b^{2}+\cos (\theta / 2) \sin (\theta / 2)(J b-b J)
$$

and that $\operatorname{tr}(J b)=\operatorname{tr}(b J)=0$. It follows that

$$
\operatorname{tr}\left({ }^{t} \beta_{\theta} \cdot \beta_{\theta}\right)=2 \cos ^{2}(\theta / 2)+\sin ^{2}(\theta / 2) \operatorname{tr}\left(b^{2}\right) .
$$

Since the eigenvalues of $b$ are in $[\epsilon, 1 / \epsilon]$, it follows that the identity between $\left(\mathbb{H}^{2}, g\right)$ and $\left(\mathbb{H}^{2}, g_{\theta}\right)$ is quasiconformal, and that $g_{\theta}$ and $g_{\theta}^{\star}$ are complete.

As a consequence we can give the definition of the action considered here. Let $\psi, \psi^{\star} \in \mathcal{Q S}$ represent points $[\psi],\left[\psi^{\star}\right] \in \mathcal{T}_{U}$. Then $\bar{\psi}:=\psi^{\star} \circ \psi^{-1}: S^{1} \rightarrow S^{1}$ is a quasisymmetric homeomorphism: let $m: \mathbb{H}^{2} \rightarrow \mathbb{H}^{2}$ be the unique quasiconformal minimal Lagrangian diffeomorphism with $\partial m=\bar{\psi}$. We can then define a bundle morphism $b: T \mathbb{H}^{2} \rightarrow T \mathbb{H}^{2}$ as above, as well as two hyperbolic metrics $g_{\theta}$ and $g_{\theta}^{\star}$ associated to $\theta$ and $b$, as in Section 3. Lemma 8.3 shows that the identity between $\left(\mathbb{H}^{2}, g_{\theta}\right)$ and $\left(\mathbb{H}^{2}, g_{\theta}^{\star}\right)$ is minimal Lagrangian. 
Since $g_{\theta}$ is hyperbolic, $\left(\mathbb{H}^{2}, g_{\theta}\right)$ is isometric to the hyperbolic plane. The identity between $\left(\mathbb{H}^{2}, g\right)$ and $\left(\mathbb{H}^{2}, g_{\theta}\right)$ therefore determines a quasiconformal diffeomorphism $\Psi_{\theta}$ between $\left(\mathbb{H}^{2}, g\right)$ and $\mathbb{H}^{2}$, well-defined up to composition on the left, with boundary value $\psi_{\theta} \in \mathcal{Q S}$. Similarly, the identity map from $\left(\mathbb{H}^{2}, g\right)$ to $\left(\mathbb{H}^{2}, g_{\theta}^{\star}\right)$ determines a quasiconformal map $\Psi_{\theta}^{\star}$ between the hyperbolic plane and itself, with boundary value $\psi_{\theta}^{\star} \in \mathcal{Q S}$. Then $\Psi_{\theta}^{\star} \circ \Psi_{\theta}^{-1}$ is a quasiconformal minimal Lagrangian diffeomorphism by Lemma 8.3. We define $\mathcal{L}$ as

$$
\mathcal{L}_{e^{i \theta}}\left([\psi],\left[\psi^{\star}\right]\right)=\left(\left[\psi_{\theta}\right],\left[\psi_{\theta}^{\star}\right]\right) .
$$

To establish a relation between $\mathcal{T}_{U}$ and $\mathcal{T}_{S}$, fix a hyperbolic metric $h_{0}$ on $S$ and a universal covering map $\mathbb{H}^{2} \cong\left(\tilde{S}, \widetilde{h}_{0}\right) \rightarrow\left(S, h_{0}\right)$, so that $\rho_{0}: \pi_{1}(S) \rightarrow \mathrm{PSL}_{2}(\mathbb{R})$ is the associated holonomy representation. Given $[h] \in \mathcal{T}_{S}$, we can consider the lift $\tilde{h}$ of $h$ to $\mathbb{H}^{2}$ and let $\rho$ be its holonomy representation. The identity map from $\left(\mathbb{H}^{2}, \tilde{h}_{0}\right)$ to $\left(\mathbb{H}^{2}, \tilde{h}\right)$ determines a quasiconformal diffeomorphism $\Psi$ of $\mathbb{H}^{2}$ to itself, with boundary value $\psi \in \mathcal{Q S}$, that conjugates the action of $\rho_{0}$ on $\mathbb{H}^{2}$ to the action of $\rho$.

Let $i_{S}: \mathcal{T}_{S} \hookrightarrow \mathcal{T}_{U}$ be the canonical embedding of Teichmüller space of $S$ in the universal Teichmüller space defined as $i_{S}([h])=[\psi]$.

Proposition 8.4 The restriction via $i_{S}$ of $\mathcal{L}$ to $\mathcal{T}_{S} \times \mathcal{T}_{S} \subset \mathcal{T}_{U} \times \mathcal{T}_{U}$ is the landslide action $L$.

Proof Let $[\psi]=i_{S}([h])$ and $\left[\psi^{\star}\right]=i_{S}\left(\left[h^{\star}\right]\right)$ be points of $i_{S}\left(\mathcal{T}_{S}\right) \subset \mathcal{T}_{U}$. Let $\rho$ and $\rho^{\star}$ be the holonomy representations of $h$ and $h^{\star}$ respectively. Then $\psi$ and $\psi^{\star}$ are the boundary values of quasiconformal maps $\Psi, \Psi^{\star}: \mathbb{H}^{2} \rightarrow \mathbb{H}^{2}$ which are conjugating $\rho_{0}$ to actions $\rho$ and $\rho^{\star}$ on $\mathbb{H}^{2}$. By construction, $g_{\theta}=\widetilde{h}_{\theta}$ is $\rho$-invariant and $g_{\theta}^{\star}=\widetilde{h}_{\theta}^{\star}$ is $\rho^{\star}$-invariant; moreover, $\Psi_{\theta}$ (resp. $\Psi_{\theta}^{\star}$ ) conjugates the action of $\rho_{0}$ to the action of the holonomy representation $\rho_{\theta}$ of $h_{\theta}$ (resp. the holonomy representation $\rho_{\theta}^{\star}$ of $h_{\theta}^{\star}$ ). Hence, $\left(\left[\psi_{\theta}\right],\left[\psi_{\theta}^{\star}\right]\right) \in i_{S}\left(\mathcal{T}_{S}\right) \times i_{S}\left(\mathcal{T}_{S}\right) \subset \mathcal{T}_{U} \times \mathcal{T}_{U}$, and the restriction of $\mathcal{L}$ to $\mathcal{T}_{S} \times \mathcal{T}_{S}$ coincides with $L$, as claimed.

Theorem 8.5 The map $\mathcal{L}$ defines a nontrivial action of $S^{1}$ on $\mathcal{T}_{U}$.

Sketch of the proof To prove that $\mathcal{L}$ determines an action of $S^{1}$, it is sufficient to check that, $\mathcal{L}_{e^{i \theta}}\left(\mathcal{L}_{e^{i \theta^{\prime}}}\left([\psi],\left[\psi^{\star}\right]\right)\right)=\mathcal{L}_{e^{i\left(\theta+\theta^{\prime}\right)}}\left([\psi],\left[\psi^{\star}\right]\right)$, for all $\theta, \theta^{\prime} \in \mathbb{R}$ and all $[\psi],\left[\psi^{\star}\right] \in \mathcal{T}_{U}$.

However, this follows from the fact that $\Psi_{\theta}^{\star} \circ \Psi_{\theta}^{-1}$ is minimal Lagrangian, followed by the same argument used in the proof of Theorem 1.8; so we do not repeat them here.

The nontriviality of $\mathcal{L}$ is clear, since $L$ is nontrivial in all the copies of Teichmüller spaces of surfaces of finite genus; see Proposition 8.4. 


\section{Applications, extensions, and questions}

This section contains a brief outline of some possible applications of the landslide flow developed here, and of some open questions.

\subsection{Holomorphic disks in Teichmüller space}

One obvious consequence of Theorems 1.15 and 5.1 is the existence of many holomorphic disks in Teichmüller space of $S$ : given $h, h^{\prime} \in \mathcal{T}$ with $h \neq h^{\prime}$ and given $\zeta \in S^{1} \backslash\{1\}$ there exists $h^{\star} \in \mathcal{T}$ and a holomorphic map $C_{\bullet}\left(h, h^{\star}\right): \bar{\Delta} \rightarrow \mathcal{T}$ from the unit disk in $\mathbb{C}$ to Teichmüller space of $S$, such that $C_{1}\left(h, h^{\star}\right)=h$ and $C_{\zeta}\left(h, h^{\star}\right)=h^{\prime}$.

It is of course conceivable that the disks obtained in this manner for two different values of $h^{\prime}$ have the same image. However, there are reasons to believe that it is not often the case. If this is correct, it would mean that the landslide disks provide a $(12 g-11)$-dimensional family of holomorphic disks in $\mathcal{T}$.

\subsection{Other questions}

There are many remaining questions concerning the landslide flow or its complex extension, mostly motivated by the analogy with the earthquake flow. Some of those statements can be translated in terms of 3-dimensional hyperbolic or AdS geometry. We give here a short list of example of possible questions.

Smooth grafting as homeomorphism Recall that Scannell and Wolf [35] proved that, for $\lambda \in \mathcal{M L}$ fixed, the map $h \mapsto g r_{\lambda}(h)$ is a homeomorphism of $\mathcal{T}$. When $h \in \mathcal{T}$ is fixed, the map $\lambda \mapsto g r_{\lambda}(h)$ is also a homeomorphism from $\mathcal{M L}$ to $\mathcal{T}$; see Dumas and Wolf [11].

It is tempting to ask whether those statements can be extended to the smooth grafting map sgr. Note that in this setting the two statements above concerning the grafting map — with the measured lamination fixed, and with the hyperbolic metric fixed - are now merged into one, since the two hyperbolic metrics that occur in the map sgr play symmetric roles.

Question 9.1 Let $s \in(0,1)$, and let $h \in \mathcal{T}$. Is the map $h^{\star} \mapsto \operatorname{sgr}_{s}\left(h, h^{\star}\right)$ a homeomorphism from $\mathcal{T}$ to $\mathcal{T}$ ?

This statement can be translated in terms of the geometry of hyperbolic ends, in the following, essentially equivalent question. 
Question 9.2 Let $h, c \in \mathcal{T}$ and let $K \in(-1,0)$.

- Is there a unique hyperbolic end with conformal structure at infinity $c$, and containing an embedded surface of constant curvature $K$ with induced metric proportional to $h$ ?

- Is there a unique hyperbolic end with conformal structure at infinity $c$, containing an embedded surface of constant curvature $K$ with third fundamental form proportional to $h^{\star}$ ?

The action of the landslide flow at infinity It is quite natural to wonder to what extend the landslide flow can be extended to Thurston boundary of Teichmüller space. One side of this question is already answered above in Section 6, concerning the limit of $L$ to the earthquake flow when one of the parameter converges to Thurston boundary and the other is fixed. However other questions can be asked, in particular in light of the results of Wolf [44] on the behavior of harmonic maps at the boundary of $\mathcal{T}$.

The landslide flow as a Hamiltonian flow Consider a fixed measured lamination $\lambda \in \mathcal{M L}$. The flow of earthquakes along $\lambda$ is the Hamiltonian flow of the length of $\lambda$, considered as a function on $\mathcal{T}$, with respect to the Weil-Petersson symplectic structure. In a similar way, is the landslide flow the Hamiltonian flow of some functional on $\mathcal{T} \times \mathcal{T}$ ?

The data at infinity of hyperbolic ends For all $K \in(-1,0)$, there is a parameterization of $\mathcal{C P}$ by $\mathcal{T} \times \mathcal{T}$, with a complex projective structure $P$ corresponding to $\left(h, h^{\star}\right)$ if the hyperbolic end $E$ with complex projective structure $P$ at infinity contains a surface of constant curvature $K$ with induced metric proportional to $h$ and third fundamental form proportional to $h^{\star}$.

There is also another parameterization of $\mathcal{C P}$ by the space of couples $(h, b)$, where $h \in \mathcal{T}$ and where $b$ is a bundle morphism which is self-adjoint for $h$ and satisfies the Codazzi equation and $\operatorname{det}(b)=1$.

Given a $\mathbb{C P} \mathbb{P}^{1}$-structure $P$, we can also consider the data at infinity $I^{*}$ and $I I I^{*}$ of the corresponding hyperbolic end, as defined by Krasnov and the third author in [22], and take the limit as $K \rightarrow 0$. Is it true that $h$ and $h^{\star}$ limit to $I^{*}$ and $I I I^{*}$ respectively? And that the traceless part of $B$, suitably renormalized, converges to $B^{*}$ ?

Landslides on the universal Teichmüller space Section 8 on the universal Teichmüller space leaves a number of elementary questions unanswered. One natural question is whether for fixed $[\psi],\left[\psi^{\star}\right] \in \mathcal{T}_{U}$ the map $e^{i \theta} \rightarrow \mathcal{L}_{e^{i} \theta}^{1}\left([\psi],\left[\psi^{\star}\right]\right)$ extends to a holomorphic disk in $\mathcal{T}_{U}$, as for the landslide action on the Teichmüller space of a closed surface. 
Another natural question is whether all fixed points of the landslide action on $\mathcal{T}_{U} \times \mathcal{T}_{U}$ are on the diagonal.

Cone singularities It appears possible that all the results obtained here extend from closed hyperbolic surfaces to finite volume hyperbolic surfaces, and more generally to hyperbolic surfaces with cone singularities (perhaps of angle less than $\pi$ ). The 3-dimensional AdS or hyperbolic part of the picture would then be filled with 3dimensional AdS or hyperbolic manifolds with "particles", as considered eg in [21], by Moroianu and the third author in [32] and by Lecuire and the third author [26].

\section{Appendix A Cyclic flow for Teichmüller space of flat 2-tori}

Let $\mathbb{T}:=\mathbb{R}^{2} / \mathbb{Z}^{2}$ be a real compact 2 -torus and denote by $\mathcal{T}(\mathbb{T})$ the space of flat metrics of area 1 on $\mathbb{T}$ up to isotopy.

It is well-known that there is an identification

$$
\begin{aligned}
\mathbb{H}^{2} \cong \mathrm{SO}_{2}(\mathbb{R}) \backslash \mathrm{SL}_{2}(\mathbb{R}) & \longrightarrow \mathcal{T}(\mathbb{T}), \\
{[M] } & \mapsto\left[g_{M}\right],
\end{aligned}
$$

where $g_{M}:=M^{*}\left(d x^{2}+d y^{2}\right)=M^{T} M$ is the flat metric,

$$
J=\left(\begin{array}{cc}
0 & -1 \\
1 & 0
\end{array}\right)
$$

and $J_{M}:=M^{-1} J M$ is the complex structure on $\mathbb{T}$ associated to $[M]$.

Notice that, given two points $\left[g_{M}\right]$ and $\left[g_{N}\right]$ in $\mathcal{T}(\mathbb{T})$, we have that the identity map $\operatorname{id}_{M, N}:\left(\mathbb{T}, g_{M}\right) \rightarrow\left(\mathbb{T}, g_{N}\right)$ is harmonic and it is a Teichmüller map. Moreover, all the other harmonic (or Teichmüller) maps are obtained by composing $\operatorname{id}_{M, N}$ with translations.

Clearly, the energy density of $\operatorname{id}_{M, N}$ is constantly

$$
e_{M, N}=\frac{1}{2} \operatorname{tr}\left[\left(M^{T} M\right)^{-1}\left(N^{T} N\right)\right]=\frac{1}{2} \operatorname{tr}\left(P^{T} P\right) \quad \text { with } P=N M^{-1},
$$

and so the energy is $E_{M, N}=e_{M, N}$.

An easy computation shows that the absolute value squared of the Beltrami differential and the quasiconformality coefficient are constantly

$$
\left|\mu_{M, N}\right|^{2}=\frac{E-1}{E+1}, \quad K_{M, N}=E+\sqrt{E^{2}-1} .
$$


At the infinitesimal level, let $P_{\epsilon}:=e^{\varepsilon X}$ with $X \in \mathfrak{s l}_{2}(\mathbb{R})$. So

$$
\begin{aligned}
E_{M, P_{\varepsilon} M} & =1+\operatorname{tr}\left[\left(\frac{X+X^{T}}{2}\right)^{2}\right] \varepsilon^{2}+o\left(\varepsilon^{2}\right), \\
\left|\mu_{M, P_{\varepsilon} M}\right| & =\sqrt{\operatorname{tr}\left[\left(\frac{X+X^{T}}{2}\right)^{2}\right]} \frac{\varepsilon}{\sqrt{2}}+o(\varepsilon), \\
K_{M, P_{\varepsilon} M} & =1+\sqrt{2 \operatorname{tr}\left[\left(\frac{X+X^{T}}{2}\right)^{2}\right]} \varepsilon+o(\varepsilon) .
\end{aligned}
$$

Hence, the tangent vector in $T_{\left[g_{M}\right]} \mathcal{T}(\mathbb{T})$ corresponding to the path $\varepsilon \mapsto P_{\varepsilon} M$, whose associated infinitesimal Beltrami differential is $\dot{\mu}=\left.(d / d \varepsilon) \mu_{M, P_{\varepsilon} M}\right|_{\varepsilon=0}$, has Teichmüller and Weil-Petersson norms equal to

$$
\|\dot{\mu}\|_{\mathcal{T}}=\|\dot{\mu}\|_{\infty}=\sqrt{\frac{1}{2} \operatorname{tr}\left[\left(\frac{X+X^{T}}{2}\right)^{2}\right]}=\left(\int_{\mathbb{T}}\|\dot{\mu}\|^{2} d A_{g_{M}}\right)^{1 / 2}=\|\dot{\mu}\|_{W P} .
$$

Now, we want to consider the Minkowski model for $\mathbb{H}^{2}$. Denote by $\mathcal{K}$ be the Killing form $\mathcal{K}(X, Y)=4 \operatorname{tr}(X Y)$ on the Lie algebra $\mathfrak{s l}_{2}(\mathbb{R})$. The map

$$
\begin{aligned}
\mathrm{SL}_{2}(\mathbb{R}) & \longrightarrow \mathfrak{s l}_{2}(\mathbb{R}), \\
M & \mapsto \frac{1}{2 \sqrt{2}} J_{M},
\end{aligned}
$$

embeds $\mathbb{H}^{2}=\mathrm{SO}_{2}(\mathbb{R}) \backslash \mathrm{SL}_{2}(\mathbb{R})$ as one of the components of the $(-1)$-sphere $\mathbb{S}(-1):=\left\{X \in \mathfrak{s l}_{2}(\mathbb{R}) \mid \mathcal{K}(X, X)=-1\right\}$.

Hence, $\mathcal{K}$ induces a scalar product $\langle\cdot, \cdot\rangle$ on $\mathbb{H}^{2}$ that can be expressed as

$$
-\cosh \left(d_{\mathbb{H}^{2}}([M],[N])\right)=\langle[M],[N]\rangle=\frac{1}{2} \operatorname{tr}\left(J_{M} J_{N}\right)=-E_{M, N},
$$

because $J Q=\operatorname{det}(Q)\left(Q^{T}\right)^{-1} J$ for every $Q \in \mathrm{GL}_{2}(\mathbb{R})$. Thus $d_{\mathbb{H}^{2}}=2 d_{\mathcal{T}}=2 d_{W P}$.

Let $h, h^{\star} \in \mathcal{T}(\mathbb{T})$ and pick $M, M^{\star} \in \mathrm{SL}_{2}(\mathbb{R})$ such that $h=g_{M}$ and $h^{\star}=g_{M^{\star}}$. Clearly, $\operatorname{id}_{M, M^{\star}}:(\mathbb{T}, h) \rightarrow\left(\mathbb{T}, h^{\star}\right)$ is minimal Lagrangian and the $g_{M}$-self-adjoint operator $b$ is

$$
b=M^{-1} \sqrt{P^{T} P} M \quad \text { with } P=M^{\star} M^{-1},
$$

where $\sqrt{P^{T} P}$ is the positive square root. Note that $\operatorname{det}(b)=1$. Then the center $c \in \mathcal{T}(\mathbb{T})$ of $\left(h, h^{\star}\right)$ corresponds to the matrix $C=1 / \sqrt{\operatorname{det}(E+b)} M(E+b)$. 
Let $\sqrt{b}$ be the positive square root of $b$, considered as a self-adjoint endomorphism for $g_{M}$-that is, $\sqrt{b}=M^{-1} \sqrt{\sqrt{P^{T} P}} M$. Since $b$ has determinant $1, \sqrt{b}$ is proportional to $E+b$. An easy computation using this fact shows that

$$
J_{M}=\sqrt{b} J_{C} \sqrt{b}^{-1}, \quad J_{M^{\star}}=\sqrt{b}^{-1} J_{C} \sqrt{b} .
$$

Since $\sqrt{b}$ is self-adjoint for $g_{M}$ and of determinant $1, J_{M} \sqrt{b}=\sqrt{b}^{-1} J_{M}$. One can also conclude that

$$
\begin{aligned}
\cosh d_{\mathbb{H}^{2}}\left([M],\left[M^{\star}\right]\right) & =\frac{1}{2} \operatorname{tr}\left(b^{2}\right), \\
\cosh d_{\mathbb{H}^{2}}([M],[C]) & =\cosh d_{\mathbb{H}^{2}}\left([C],\left[M^{\star}\right]\right)=\frac{1}{2} \operatorname{tr}(b),
\end{aligned}
$$

and so $d_{\mathbb{H}^{2}}\left([M],\left[M^{\star}\right]\right)=2 \log \kappa$ and $d_{\mathbb{H}^{2}}([M],[C])=d_{\mathbb{H}^{2}}\left([C],\left[M^{\star}\right]\right)=\log \kappa$, where $\kappa \geq 1$ is the larger eigenvalue of $b$. Hence, $[C] \in \mathbb{H}^{2}$ is the midpoint between $[M]$ and $\left[M^{\star}\right]$.

As $J_{C}=J_{M} b$, the matrix corresponding to $h_{\theta}$ is

$$
M_{\theta}=M\left(\cos (\theta / 2) E+\sin (\theta / 2) J_{M} b\right)=M e^{(\theta / 2) J_{C}},
$$

where $M e^{(\theta / 2) J_{C}}$ is the matrix associated to the point of $\mathbb{H}^{2}$ obtained by rotating $[M]$ of an angle $\theta$ about the center $[C]$.

This shows that the cyclic flow on $\mathcal{T}(\mathbb{T}) \times \mathcal{T}(\mathbb{T}) \cong \mathbb{H}^{2} \times \mathbb{H}^{2}$ is the rotation about the center.

\section{References}

[1] S I Al'ber, Spaces of mappings into a manifold of negative curvature, Dokl. Akad. Nauk SSSR 178 (1968) 13-16 MR0230254 In Russian; translated in Soviet Math. Dokl. 9 (1968) 6-9

[2] L Andersson, T Barbot, R Benedetti, F Bonsante, W M Goldman, F Labourie, K P Scannell, J-M Schlenker, Notes on: "Lorentz spacetimes of constant curvature" [Geom. Dedicata 126 (2007), 3-45; MR2328921] by G. Mess, Geom. Dedicata 126 (2007) 47-70 MR2328922

[3] T Barbot, F Béguin, A Zeghib, Constant mean curvature foliations of globally hyperbolic spacetimes locally modelled on $\mathrm{AdS}_{3}$, Geom. Dedicata 126 (2007) 71-129 MR2328923

[4] T Barbot, F Béguin, A Zeghib, Prescribing Gauss curvature of surfaces in 3dimensional spacetimes: application to the Minkowski problem in the Minkowski space, Ann. Inst. Fourier (Grenoble) 61 (2011) 511-591 MR2895066 
[5] M Belraouti, Sur la géométrie de la singularité initiale des espaces-temps plats globalement hyperboliques arXiv:1201.3716

[6] M Belraouti, personal communication on work in progress (2012)

[7] R Benedetti, F Bonsante, Canonical Wick rotations in 3-dimensional gravity, Mem. Amer. Math. Soc. 198 (2009) viii+164 MR2499272

[8] R Benedetti, E Guadagnini, Cosmological time in $(2+1)$-gravity, Nuclear Phys. B 613 (2001) 330-352 MR1857817

[9] F Bonsante, J-M Schlenker, Maximal surfaces and the universal Teichmüller space, Invent. Math. 182 (2010) 279-333 MR2729269

[10] J Douglas, Minimal surfaces of higher topological structure, Ann. of Math. 40 (1939) 205-298 MR1503457

[11] D Dumas, M Wolf, Projective structures, grafting and measured laminations, Geom. Topol. 12 (2008) 351-386 MR2390348

[12] J Eells, Jr, J H Sampson, Harmonic mappings of Riemannian manifolds, Amer. J. Math. 86 (1964) 109-160 MR0164306

[13] A E Fischer, A J Tromba, A new proof that Teichmüller space is a cell, Trans. Amer. Math. Soc. 303 (1987) 257-262 MR896021

[14] F P Gardiner, W J Harvey, Universal Teichmüller space, from: "Handbook of complex analysis: geometric function theory, Vol. 1”, (R Kühnau, editor), North-Holland, Amsterdam (2002) 457-492 MR1966201

[15] P Hartman, On homotopic harmonic maps, Canad. J. Math. 19 (1967) 673-687 MR0214004

[16] C D Hodgson, S P Kerckhoff, Rigidity of hyperbolic cone-manifolds and hyperbolic Dehn surgery, J. Differential Geom. 48 (1998) 1-59 MR1622600

[17] C D Hodgson, I Rivin, A characterization of compact convex polyhedra in hyperbolic 3-space, Invent. Math. 111 (1993) 77-111 MR1193599

[18] H Jacobowitz, The Gauss-Codazzi equations, Tensor 39 (1982) 15-22 MR836907

[19] J Jost, Two-dimensional geometric variational problems, Pure and Applied Mathematics (New York), John Wiley \& Sons Ltd., Chichester (1991) MR1100926

[20] S P Kerckhoff, The Nielsen realization problem, Ann. of Math. 117 (1983) 235-265 MR690845

[21] K Krasnov, J-M Schlenker, Minimal surfaces and particles in 3-manifolds, Geom. Dedicata 126 (2007) 187-254 MR2328927

[22] K Krasnov, J-M Schlenker, On the renormalized volume of hyperbolic 3-manifolds, Comm. Math. Phys. 279 (2008) 637-668 MR2386723

[23] R S Kulkarni, U Pinkall, A canonical metric for Möbius structures and its applications, Math. Z. 216 (1994) 89-129 MR1273468 
[24] F Labourie, Problème de Minkowski et surfaces à courbure constante dans les variétés hyperboliques, Bull. Soc. Math. France 119 (1991) 307-325 MR1125669

[25] F Labourie, Surfaces convexes dans l'espace hyperbolique et $\mathbf{C P}^{1}$-structures, J. London Math. Soc. 45 (1992) 549-565 MR1180262

[26] C Lecuire, J-M Schlenker, The convex core of quasifuchsian manifolds with particles arXiv:0909.4182

[27] B Maskit, Comparison of hyperbolic and extremal lengths, Ann. Acad. Sci. Fenn. Ser. A I Math. 10 (1985) 381-386 MR802500

[28] C T McMullen, Complex earthquakes and Teichmüller theory, J. Amer. Math. Soc. 11 (1998) 283-320 MR1478844

[29] G Mess, Lorentz spacetimes of constant curvature, Geom. Dedicata 126 (2007) 3-45 MR2328921

[30] Y N Minsky, The classification of punctured-torus groups, Ann. of Math. 149 (1999) 559-626 MR1689341

[31] G Mondello, Flows of $S L_{2}(R)$-type on the cotangent space to Teichmüller space, in preparation

[32] S Moroianu, J-M Schlenker, Quasi-Fuchsian manifolds with particles, J. Differential Geom. 83 (2009) 75-129 MR2545031

[33] J H Sampson, Some properties and applications of harmonic mappings, Ann. Sci. École Norm. Sup. 11 (1978) 211-228 MR510549

[34] K P Scannell, Flat conformal structures and the classification of de Sitter manifolds, Comm. Anal. Geom. 7 (1999) 325-345 MR1685590

[35] K P Scannell, M Wolf, The grafting map of Teichmüller space, J. Amer. Math. Soc. 15 (2002) 893-927 MR1915822

[36] J-M Schlenker, Métriques sur les polyèdres hyperboliques convexes, J. Differential Geom. 48 (1998) 323-405 MR1630178

[37] J-M Schlenker, Hypersurfaces in $H^{n}$ and the space of its horospheres, Geom. Funct. Anal. 12 (2002) 395-435 MR1911666

[38] R M Schoen, The role of harmonic mappings in rigidity and deformation problems, from: "Complex geometry", (G Komatsu, Y Sakane, editors), Lecture Notes in Pure and Appl. Math. 143, Dekker, New York (1993) 179-200 MR1201611

[39] R Schoen, S T Yau, On univalent harmonic maps between surfaces, Invent. Math. 44 (1978) 265-278 MR0478219

[40] M Spivak, A comprehensive introduction to geometry, Vol. I-V, Publish or Perish (1970)

[41] K Tenenblat, On isometric immersions of Riemannian manifolds, Bol. Soc. Brasil. Mat. 2 (1971) 23-36 MR0328832 
[42] W P Thurston, The geometry and topology of three-manifolds, Princeton Univ. Math. Dept. Lecture Notes (1979) Available at http://msri.org/publications/books/ gt $3 \mathrm{~m} /$

[43] M Wolf, The Teichmüller theory of harmonic maps, J. Differential Geom. 29 (1989) 449-479 MR982185

[44] M Wolf, Infinite energy harmonic maps and degeneration of hyperbolic surfaces in moduli space, J. Differential Geom. 33 (1991) 487-539 MR1094467

[45] M Wolf, Harmonic maps from surfaces to $\mathbf{R}$-trees, Math. Z. 218 (1995) 577-593 MR1326987

Dipartimento do Matematica, Università degli Studi di Pavia Via Ferrata, 1, I-27100 Pavia, Italy

Università di Roma "La Sapienza”, Dipartimento di Matematica “Guido Castelnuovo" Piazzale Aldo Moro 5, I-00185 Roma, Italy

Institut de Mathématiques de Toulouse, Université Paul Sabatier 118 route de Narbonne, F-31062 Toulouse Cedex 9, France francesco.bonsante@unipv.it, mondello@mat.uniroma1.it, schlenker@math.univ-toulouse.fr

http://www-dimat.unipv.it/ bonsante/, http://www.mat.uniroma1.it/ people/mondello/, http://www.math.univ-toulouse.fr/ schlenker/

Proposed: Benson Farb

Seconded: Walter Neumann, David Gabai

Received: 6 September 2011 Revised: 5 July 2012 